\title{
THE VALUATION OF A NATURAL GAS-FIRED POWER PLANT WITH MULTIPLE TURBINES USING CLEAN SPARK SPREAD AND WEATHER OPTIONS
}

\author{
by \\ M. Reaz-us Salam Elias \\ M.Eng., Asian Institute of Technology, Bangkok, Thailand, 1999
}

B.Sc. (Mechanical Eng.), Rajshahi University of Engineering and Technology, Bangladesh, 1995

\author{
A dissertation \\ presented to Ryerson University \\ in partial fulfillment of the \\ requirements for the degree of \\ Doctor of Philosophy \\ in the Program of \\ Mechanical and Industrial Engineering
}

Toronto, Ontario, Canada, 2015

(C)M. R. S. Elias 2015 


\section{AUTHOR'S DECLARATION FOR ELECTRONIC SUBMISSION OF A DISSERTATION}

I hereby declare that I am the sole author of this dissertation. This is a true copy of the dissertation, including any required final revisions, as accepted by my examiners.

I authorize Ryerson University to lend this dissertation to other institutions or individuals for the purpose of scholarly research.

I further authorize Ryerson University to reproduce this dissertation by photocopying or by other means, in total or in part, at the request of other institutions or individuals for the purpose of scholarly research.

I understand that my dissertation may be made electronically available to the public. 
The Valuation of a Natural Gas-fired Power Plant with Multiple Turbines Using Clean Spark Spread and Weather Options

Doctor of Philosophy 2015

M. Reaz-us Salam Elias

Mechanical and Industrial Engineering

Ryerson University

\begin{abstract}
Assessing the value of a power plant is an important issue for plant owners and prospective buyers. In a deregulated market, an owner has the option to operate the plant when the revenue from selling the electricity is higher than the cost of operating the plant. This option is known as the spark spread option. Under emission restrictions, when the carbon cost is deducted from the spark spread, the option is named as the clean spark spread option. This thesis presents an analysis on the spark spread and clean spark spread option based valuation methods for a power plant with multiple gas turbines having different input-output characteristics, emission rates, and capacities. Electricity, natural gas and carbon allowance prices are assumed to follow mean-reverting processes. Results demonstrate that $\mathrm{CO}_{2}$ allowance cost reduces the expected plant value, while the flexibility of switching among turbines adds value to the power plant.

Weather also affects the power plant operation. This thesis also presents a valuation model for a power plant integrating spark spread and weather options. A cooler winter drawing more electricity could generate a higher payoff for the plant owner. A warmer winter, however, could lead to a lower payoff. An owner holding a long position in a temperature-based put option could exercise the option when the winter is milder. The exercise is triggered by the drop of heating degree days below a strike degree day. The number of weather contracts to buy is determined by minimizing the variance of the total payoff. Pricing of the weather option is calculated based on the mean-reverting behavior of temperature. Results demonstrate that the integrating weather option along with spark spread option adds value to the downward spark spread option based valuation of the plant in a warmer winter.

A comparison of temperature modeling approaches with an aim to pricing weather option is also investigated. Regime-switching models generated from a combination of different underlying processes are utilized to determine the expected heating and cooling degree days. Weather option prices are then calculated based on a range of strike heating degree days.
\end{abstract}




\section{Acknowledgements}

I am grateful to my supervisors, Dr. M. Wahab M. Ismail and Dr. Liping Fang, Department of Mechanical and Industrial Engineering, Ryerson University, Toronto, Canada. Both of them have distinct excellent qualities. Their critical thinking in doing research, supervision and guidance in achieving a goal are sources of my valuable experience and nice memories. I have learned a lot from them.

I am thankful to the committee members, Dr. Gordon Sick, Professor of Finance, University of Calgary, Canada, Dr. Paul S. Moore, Department of Sociology, Ryerson University, Dr. Arnold Yuan, Department of Civil Engineering, Ryerson University, and Dr. Saeed Zolfaghari, Dr. Cory Searcy, and Dr. Ahmad Gashempoor of Mechanical and Industrial Engineering Department, Ryerson University, Toronto, Canada, for their valuable comments and suggestions that improve the exposition of the thesis. I also thank all other faculty and staff members of the Department of Mechanical and Industrial Engineering, Ryerson University, Toronto, Canada for their cordial help and support.

Debt to parents is unpayable and so do I. No words of acknowledgement suffice their selfless sacrifices for me. I express my gratitude to my wife, sons and daughter for enduring the 'entropy' of the PhD research from time to time. 


\section{Table of Contents}

1 Introduction $\quad 1$

1.1 The valuation of a power plant $\ldots \ldots \ldots \ldots \ldots \ldots \ldots$

1.2 Research motivation . . . . . . . . . . . . . . . . . . . 2

1.3 Research objectives . . . . . . . . . . . . . . . . . . 3

1.4 Research contributions . . . . . . . . . . . . . . . . . . 3

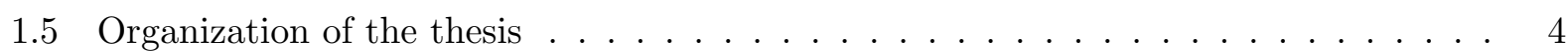

2 Literature Review 5

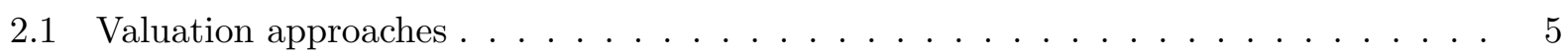

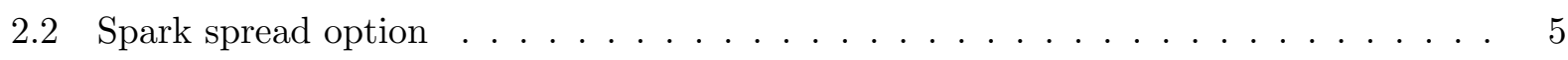

2.3 The valuation based on spark spread option $\ldots \ldots \ldots \ldots \ldots$

2.4 The carbon emission market $\ldots \ldots \ldots \ldots \ldots \ldots \ldots$

2.5 The valuation based on clean spark spread option . . . . . . . . . . . . . . . 10

2.6 Weather derivatives $\ldots \ldots \ldots \ldots \ldots \ldots \ldots \ldots$

2.7 Research gaps . . . . . . . . . . . . . . . . . . . . . . . . . . 14

3 Methodology 16

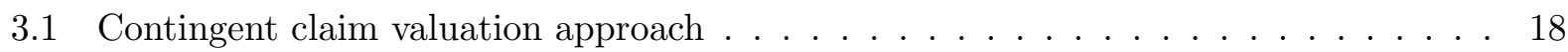

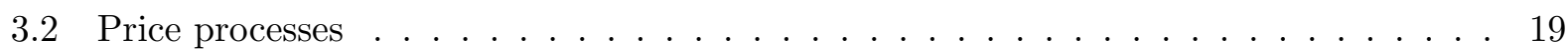

3.3 Lattice representation of price processes $\ldots \ldots \ldots \ldots \ldots \ldots$

3.3.1 A bivariate lattice for spark spread option . . . . . . . . . . . . . . . . 21

3.3.2 A trivariate lattice for clean spark spread option . . . . . . . . . . . . . 23

3.4 Regime-switching models . . . . . . . . . . . . . . . . . . . 24

3.5 Dynamic programming approach . . . . . . . . . . . . . . 25

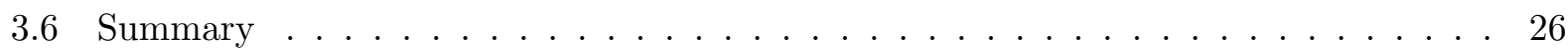

4 The Valuation of a Power Plant with Multiple Turbines using Spark Spread and Clean Spark Spread Options 


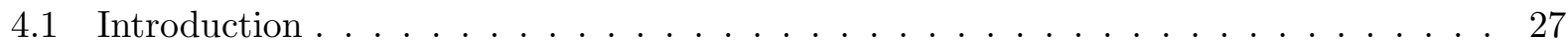

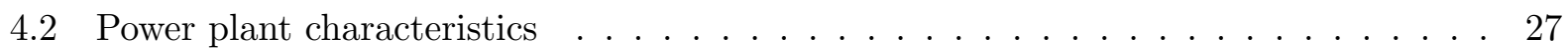

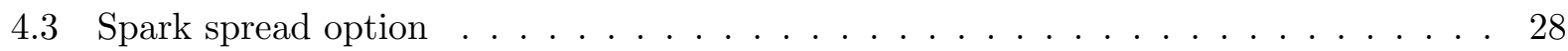

4.4 Clean spark spread option . . . . . . . . . . . . . . . . . . . . . . . 29

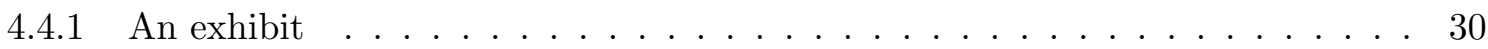

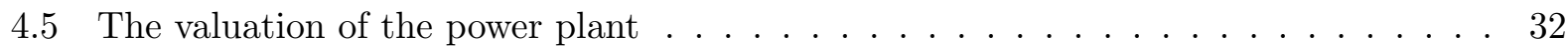

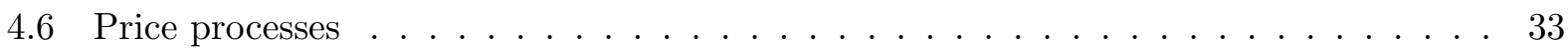

4.6.1 An estimation of mean-reverting process parameters . . . . . . . . . . 34

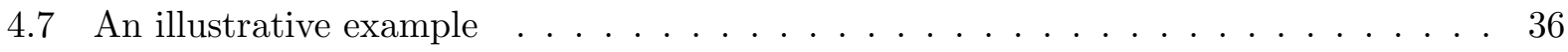

4.8 Numerical results . . . . . . . . . . . . . . . . . . . . . . . . . . 38

4.8.1 Expected spark and clean spark spread option values . . . . . . . . . . . . . 38

4.8 .2 Sensitivity to price volatilities . . . . . . . . . . . . . . 41

4.8.3 Sensitivity to the mean-reverting speed of price . . . . . . . . . . . . . 45

4.8.4 Sensitivity to changes in the minimum capacities of turbines . . . . . . . . 47

4.9 Summary . . . . . . . . . . . . . . . . . . . . . . . 48

5 Integrating Weather Options in the Valuation of a Power Plant with Multiple $\begin{array}{ll}\text { Turbines } & 49\end{array}$

5.1 Introduction . . . . . . . . . . . . . . . . . . . . . . . . 49

5.2 Integrating spark spread and weather options . . . . . . . . . . . . . . . . 49

5.2 .1 The spark spread option . . . . . . . . . . . . . . . . . 50

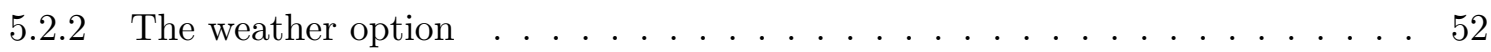

5.2 .3 Pricing weather derivatives $\ldots \ldots \ldots \ldots \ldots \ldots \ldots \ldots$

5.2 .4 Number of contracts . . . . . . . . . . . . . . . . . . . 54

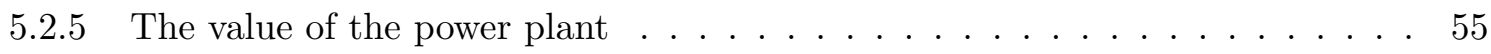

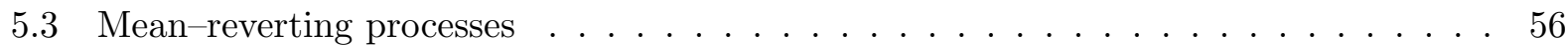

5.3.1 An estimation of mean-reverting process parameters . . . . . . . . . 57

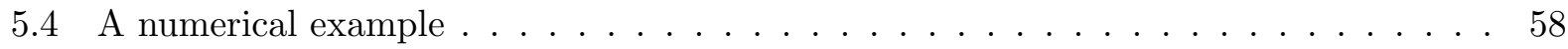

5.4 .1 Lattice construction . . . . . . . . . . . . . . . . . . 58

5.4 .2 Number of contracts . . . . . . . . . . . . . . . . . . . . 58

5.4 .3 Weather option payoff $\ldots \ldots \ldots \ldots \ldots$

5.5 Numerical results . . . . . . . . . . . . . . . . . . . . . . . 60

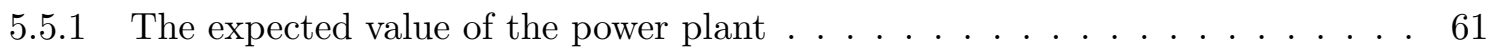

5.5.2 Sensitivity of the expected value to changes in the volatilities . . . . . . . 63

5.5.3 Sensitivity of the expected value to changes in the correlation coefficient . . . 64 
5.5.4 Sensitivity of the expected value to market price of risk for temperature . . . 65

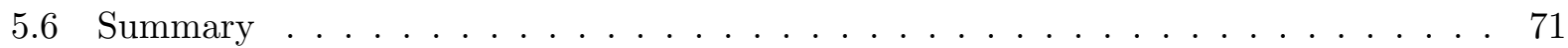

6 The Valuation of Temperature-based Weather Options $\quad 73$

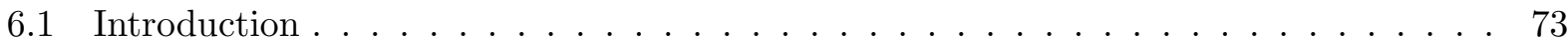

6.2 Two-state regime-switching models for Toronto temperature data . . . . . . . . . . . 74

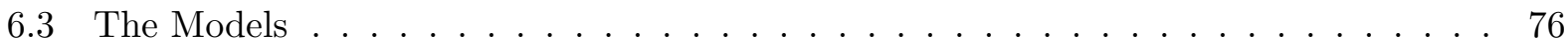

6.3.1 Model 1: With a mean-reverting process and a Brownian motion . . . . . . . 76

6.3.2 Model 2: With two Brownian motions . . . . . . . . . . . . . . . . . . . 79

6.3.3 Model 3: With two mean-reverting processes . . . . . . . . . . . . . . 80

6.3.4 Model 4: With a single mean-reverting process . . . . . . . . . . . . 80

6.4 Numerical examples . . . . . . . . . . . . . . . . . . . . . . . . . . 81

6.4.1 Model 1: With a mean-reverting process and a Brownian motion . . . . . . . 81

6.4.2 Model 2: With two Brownian motions . . . . . . . . . . . . . . . . 84

6.4.3 Model 3: With two mean-reverting processes . . . . . . . . . . . . . . 85

6.4.4 Model 4: With a single mean-reverting process . . . . . . . . . . . . 85

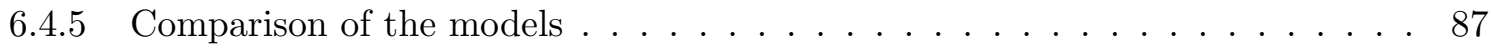

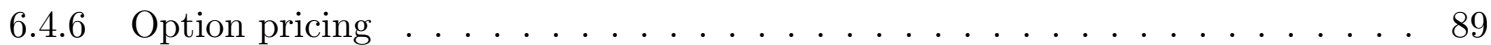

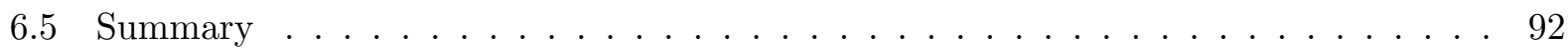

7 Conclusion and Future Work $\quad 93$

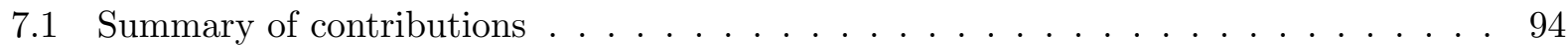

7.2 Challenges, limitations and future work . . . . . . . . . . . . . . 95

$\begin{array}{ll}\text { Appendices } & 98\end{array}$

$\begin{array}{ll}\text { Appendix A The Mean Reverting Process } & 98\end{array}$

A.1 The Mean and Variance of the Process . . . . . . . . . . . . . . . . 98

$\begin{array}{ll}\text { Appendix B Building a Bivariate Lattice } & 101\end{array}$

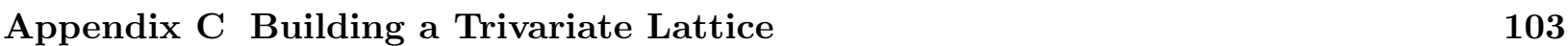

Appendix D Discretizing the Orenstein-Uhlenbeck Process in an AR Process 107

Appendix E Step size and Branch Probabilities by Matching the Moments 108

$\begin{array}{ll}\text { References } & 110\end{array}$ 


\section{List of Tables}

2.1 A review of literature on the valuation of natural gas-fired power plants $\ldots \ldots$. . . 9

2.2 Summary of approaches to pricing weather derivatives $\ldots \ldots \ldots \ldots \ldots$

4.1 Turbine characteristics of a power plant . . . . . . . . . . . . . . . 30

4.2 Mean-reverting parameters for price processes . . . . . . . . . . . . . 35

4.3 Geometric Brownian motion parameters for $\mathrm{CO}_{2}$ allowance price . . . . . . . . . . 35

4.4 Turbine startup and shutdown costs in Euro . . . . . . . . . . . . . . 38

4.5 Expected SS and CSS values with and without flexibilities at different states of the power plant . . . . . . . . . . . . . . . . . . . . . . . 39

5.1 Mean-reverting parameters for price processes . . . . . . . . . . . . . . 57

6.1 Coefficients of $S_{t}$, the deterministic component of Toronto average daily temperature 76

6.2 Estimated regime coefficients for Model $1 \ldots \ldots \ldots$. . . . . . . . . 82

6.3 Regime parameters for Model $1 \ldots \ldots \ldots \ldots$. . . . . . . . . . 82

6.4 Branch probabilities for the mean-reverting process in Model 1 . . . . . . . . . . 82

6.5 Estimated regime coefficients for Model $2 \ldots \ldots \ldots$. . . . . . . . . . . 84

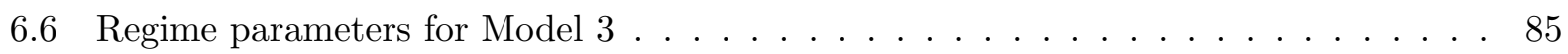

6.7 Branch probabilities of the binomial lattice . . . . . . . . . . . . 86 


\section{List of Figures}

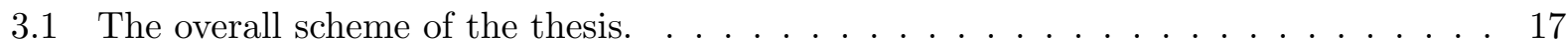

3.2 A lattice representing correlated movement of two variables. . . . . . . . . . . . . 21

3.3 A lattice representing correlated movement of three variables. . . . . . . . . . . . . 23

4.1 (a) Input-output characteristic curves and (b) corresponding average heat rates of

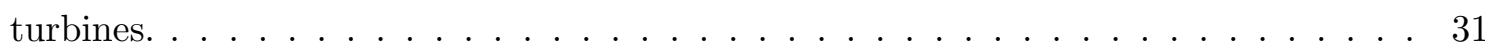

4.2 Variations in generation of power (MW) from two turbines in absence and presence

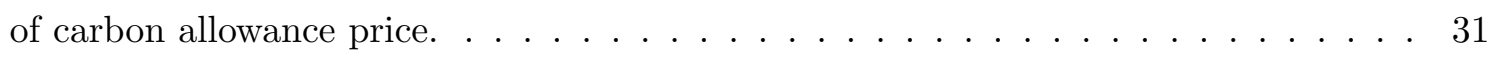

4.3 (a) Spark spread, $\Omega$, and (b) clean spark spread, $\Omega_{c}$, profits; (c) Spark spread, $\pi$, and (d) clean spark spread, $\pi_{c}$, options under different operating modes of turbines. 32

4.4 Futures prices (daily): (a) electricity, (b) natural gas and (c) carbon allowance. . . . 34

4.5 An example of movements of natural gas prices and electricity prices along a bivariate

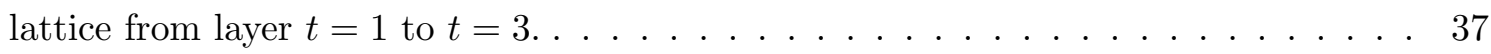

4.6 Values of the spark spread options at different states of the power plant corresponding to the prices in Figure $4.5 \ldots \ldots \ldots \ldots \ldots \ldots$

4.7 Expected spark spread option values of the power plant at different states of the power plant corresponding to the values in Figure 4.6 . . . . . . . . . . . . . 37

4.8 Expected spark and clean spark spread values of the plant at different operating states of the plant: (a) without flexibility and (b) with flexibility. . . . . . . . . . 39

4.9 Expected spark spread option based values of the power plant having two identical gas turbines similar to Turbine $1 \ldots \ldots \ldots$. . . . . . . . . . . 40

4.10 Expected clean spark spread values using two different carbon price modeling approaches: (a) without flexibility, and (b) with flexibility. . . . . . . . . . . . 41

4.11 Expected spark and clean spark spread values of the power plant at different volatilities of electricity price: (a) State 2 (without carbon cost), (b) State 2 (with carbon cost), (c) State 3 (without carbon cost), (d) State 3 (with carbon cost), (e) State 4 (without carbon cost), and (f) State 4 (with carbon cost) . . . . . . . . . . . 
4.12 Expected clean spark spread with respect to $\mathrm{CO}_{2}$ price processes: (a) without flexibility, and (b) with flexibility. . . . . . . . . . . . . . . . 42

4.13 Expected spark and clean spark spread values of the power plant due to changes in the natural gas price volatility: (a) State 2 (without carbon cost), (b) State 2 (with carbon cost), (c) State 3 (without carbon cost), (d) State 3 (with carbon cost), (e) State 4 (without carbon cost), and (f) State 4 (with carbon cost). . . . . . . . .

4.14 Expected clean spark spread value with respect to changes in the $\mathrm{CO}_{2}$ price volatility: (a) State 2 (without flexibility), (b) State 2 (with flexibility), (c) State 3 (without flexibility), (d) State 3 (with flexibility), (e) State 4 (without flexibility), and (f) State 4 (with flexibility) . . . . . . . . . . . . . . . . 44 44

4.15 Expected spark and clean spark spread values due to changes in the mean-reverting speed of electricity price: (a) State 2 (without carbon cost), (b) State 2 (with carbon cost), (c) State 3 (without carbon cost), (d) State 3 (with carbon cost), (e) State 4 (without carbon cost), and (f) State 4 (with carbon cost) . . . . . . . . . . . .

4.16 Expected spark and clean spark spread values due to changes in the mean-reverting speed of natural gas price: (a) State 2 (without carbon cost), (b) State 2 (with carbon cost), (c) State 3 (without carbon cost), (d) State 3 (with carbon cost), (e) State 4 (without carbon cost), and (f) State 4 (with carbon cost) . . . . . . . . . . 46

4.17 Expected spark and clean spark spread values with respect to the changes in the minimum capacities of turbines. . . . . . . . . . . . . . .

5.1 The variations of the adjusting factor, $\zeta\left(Q_{g}^{e}\right)$, and the optimal feasible operating quantity, $\hat{q}_{g}$, with respect to environment temperature and prices at different $R^{c}$. . 51

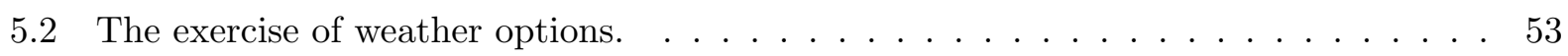

5.3 Futures prices of electricity in CAD/MWh (top) and natural gas futures prices in CAD/MMBtu (bottom) from European Energy Exchange (EEX) scaled to Canadian dollar using corresponding daily exchange rates from Euro to CAD. . . . . . . . . 56

5.4 Corresponding daily mean temperature for the city of Toronto, Canada. . . . . . . . 57

5.5 (Top) The lattice showing movements of natural gas price, electricity price and temperature. (Bottom) The corresponding values of spark spread at different states of the plant and accumulated HDD . . . . . . . . . . . . . . . . . 59

5.6 Spark spread option payoffs at State 2 of the power plant at $t=7 \ldots \ldots$. . . . 60

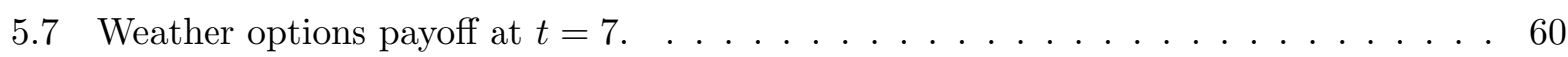

5.8 Expected value of the power plant with and without weather options at different expected HDD in the month of January: (a) State 2, (b) State 3, and (c) State 4. . 
5.9 The expected values of the power plant with and without weather options at different expected HDD in the month of February: (a) State 2, (b) State 3, and (c) State 4. . 63

5.10 The expected values of the power plant with and without weather options at different expected HDDs over the winter season combining the months of January and February: (a) State 2, (b) State 3, and (c) State 4. . . . . . . . . . . . . . . 6 (

5.11 Expected value of the power plant with and without weather options at different values of $R^{c}$ : (a) State 2, (b) State 3, and (c) State 4. . . . . . . . . . .

5.12 Sensitivity of the expected value of the power plant to the changes in electricity price volatility: (a) State 2, (b) State 3, and (c) State $4 \ldots \ldots$. . . . . . . . . 66

5.13 Sensitivity of the expected value of the power plant to the changes in natural gas price volatility: (a) State 2, (b) State 3, and (c) State $4 \ldots \ldots$. . . . . . . . 67

5.14 Sensitivity of the expected value of the power plant to the changes in temperature volatility: (a) State 2, (b) State 3, and (c) State 4. . . . . . . . . . . . . . 68

5.15 The expected value of the power plant with respect to the correlation coefficient between electricity price and temperature: (a) State 2, (b) State 3, and (c) State 4. .

5.16 The expected value of the power plant with respect to the correlation coefficient between natural gas price and temperature: (a) State 2, (b) State 3, and (c) State 4.70

5.17 Expected value of the power plant with respect to the market price of risk for temperature.

6.1 (Top) Average daily temperature (in Celcius), $Q_{t}$, with seasonality, $S_{t}$.

(Bottom) Deseasonalized stochastic component, $w_{t} \ldots \ldots \ldots \ldots \ldots \ldots$. . . . . 75

6.2 The smoothed probability of being in two states. . . . . . . . . . . . . . 76

6.3 (a) A pentanomial lattice (for a mean-reverting process and a Brownian motion) for the stochastic component of temperature. (b) The corresponding pentanomial

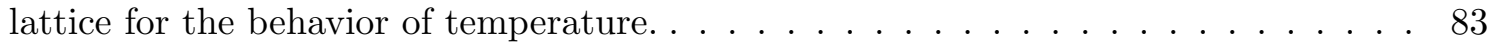

6.4 A pentanomial lattice (for a mean-reverting process and a Brownian motion) for the

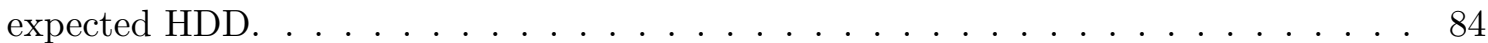

6.5 (a) A binomial lattice for the stochastic component of temperature. (b) The corre-

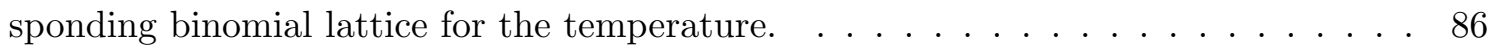

6.6 A binomial lattice for the expected HDD . . . . . . . . . . . . . . . . . 86

6.7 Comparison of the actual monthly HDD with the expected monthly HDD. . . . . . . 87

6.8 Comparison of the actual monthly CDD with the expected monthly CDD. . . . . . . 88

6.9 Comparison of the actual seasonal HDD with the expected seasonal HDD. . . . . . . 88

6.10 Comparison of the actual seasonal CDD with the expected seasonal CDD. . . . . . 88 
6.11 (a) Monthly and (b) seasonal option prices for a range of strike HDD. . . . . . . . . 90

6.12 Option prices at different market prices of risk . . . . . . . . . . . . . . . 91 


\section{Chapter 1}

\section{Introduction}

\subsection{The valuation of a power plant}

Electricity is an essential commodity in our daily life. Electricity generation in the world is expected to grow more than 90\% from 20.2 trillion kilowatthour (TkWh) in 2010 to $39.0 \mathrm{TkWh}$ in 2040 (EIA 2013). The escalation of electricity demand is stimulated by a combination of growing population, economic growth and increasing use of electrical appliances.

A power plant produces electricity by converting either fossil fuels (e.g., coal, petroleum and natural gas), nuclear energy, or natural sources of energy (e.g., water, wind and solar radiation) and sells electricity in markets. A natural gas-fired power plant burns natural gas to produce electricity and bears price risks from both purchasing natural gas and selling electricity. Until the 1990s, most power plants had been state-owned and allowed to earn a return above the cost of generation, transmission, and distribution. Deregulation, in many countries, restructured the monopolistic electricity market and has brought competition and price uncertainties for power plant owners. In a competitive electricity market, it is important to know the value of a power plant.

Valuation is the process of estimating the market-value of an asset. The market-value is the price at which an asset can be traded in a competitive setting. An appraisal of the marketvalue of a power plant is a crucial aspect in the electric power industry. Such an assessment or valuation renders a reference point for prospective owners, buyers and sellers and reduces the risk of overpaying or underselling (Wang and Min 2013). Moreover, to mitigate the financial risk, the power plant owner/operator has to evaluate the value of the plant, know the status and decide whether to run or shut down the plant or whether to switch the operating mode of the plant (Deng and Oren 2006). However, this value may not always necessarily reflect the theoretically optimal operations of the power plant, rather might refer to a usual mode of operations used by practitioners in such utilities (Wang and Min 2013). 


\subsection{Research motivation}

Buying and selling of a power plant is a frequent phenomenon in a deregulated competitive electricity market. The bankruptcy of two energy companies, Enron in 2001 and Calpine Corporation in 2005, results in numerous power plant sales and purchases (Wang and Min 2013). As the world wide electricity demand is expected to increase, buying and selling of power plants are also expected to be more frequent in the future. The value of a power plant indicates how well it performs in an uncertain competitive market. Therefore, assessing a fair value of a power plant is highly desirable by power plant owners, buyers and sellers. The work contained in this thesis is motivated by the following aspects.

Firstly, a power plant can possess one or multiple power generating units. According to the US Energy Information Administration, there are approximately 19,000 individual generators at around 7,000 power plants in the US (EIA 2015). Studies in the literature (Gardner and Zhuang 2000; Heydari and Siddiqui 2010; Wang and Min 2013) consider power plants with a single turbine operating at a constant heat rate. Moreover, recent restrictions on $\mathrm{CO}_{2}$ emissions have forced power plant operators to include emission costs in valuing power plants. Four Canadian provinces, for example, Ontario, Quebec, Manitoba, and British Columbia, along with seven U.S. states have initiated Western Climate Initiative (WCI) in order to implement a cap-and-trade scheme from January, 2012. The initial aim is to reduce emission by $15 \%$ by 2020 from the emission level of 2005 (Mnif and Davison 2011). Since 2013, power generators in many countries in Europe must purchase carbon allowances for their $\mathrm{CO}_{2}$ emissions (Heydari and Siddiqui 2010; Abadie 2015). It would be an inquisitive study to analyze the value of a natural gas-fired power plant with multiple turbines, each of them having different power generation capacities, different input-output characteristics, and emission rates in the presence and absence of stochastic carbon emission prices.

Secondly, a power plant is a weather sensitive industry. Whims of weather affect the profitability of such utilities. A gas turbine usually performs better in a cooler environment. As the environment temperature increases, the power output from a gas turbine tends to drop (Sa and Zubaidy 2011). Moreover, during a cold winter day, an increased heating requirement would induce an increased power generation from the power plant, which in turn, could produce a higher payoff. However, if the winter is mild, a lower heating requirement would tend to decrease the payoff. Through weather derivatives, a power plant can transfer its weather related uncertainties to a financial firm willing to absorb the risk. This risk is contingent on a predetermined underlying weather variable, for example, heating (cooling) degree days, HDD (CDD) measured by summing up temperature degrees below (above) a base temperature, usually $18^{\circ} \mathrm{C}$ over a contract period. An interesting research topic would be to investigate how the value of a power plant is affected by an integration 
of weather option into the spark spread option.

Thirdly, in order to integrate weather option into a spark spread option based valuation model, the pricing of weather option itself is an important issue. This thesis also analyzes temperature dynamics with an aim to pricing the temperature-based weather option.

\subsection{Research objectives}

Against the backdrop of the research motivation, the aim of this thesis is to assess the value of a natural gas-fired power plant with multiple turbines utilizing carbon emission allowance price and weather derivatives. The main objectives of the research are:

- to examine how operational flexibility of having multiple turbines affects the value of a natural gas-fired power plant in presence and absence of carbon allowance prices.

- to determine the value of a power plant integrating spark spread and weather options.

- to model the stochastic behavior of temperature with an aim to the valuation of temperaturebased weather option.

\subsection{Research contributions}

This thesis is expected to add values to the current valuation models of power plants, at least, in three aspects:

- While the valuation models in the existing literature assume a power plant with a single operating turbine which has a constant average heat rate, this study assesses the value of a natural gas-fired power plant with the operational flexibility of having multiple turbines with different heat rates, emission rates and generation capacities in presence and absence of carbon emission restriction. Results demonstrate that the $\mathrm{CO}_{2}$ allowance cost reduces the expected plant value, while the operational flexibility of switching among turbines in response to price movements adds value to the power plant. Sensitivity analyses on the parameters of electricity, gas and carbon price processes are also presented. A comparison between the effect of modeling $\mathrm{CO}_{2}$ prices as a mean-reverting and a geometric Brownian motion shows that the expected value of the power plant is higher when $\mathrm{CO}_{2}$ prices are assumed to follow a geometric Brownian motion in comparison to the expected value when $\mathrm{CO}_{2}$ prices are modeled as a mean-reverting process. The reason is that the volatility of a geometric Brownian motion grows infinitely while the volatility of a mean-reverting process is bounded and decays in the long run to a value proportional to the ratio of the variance and the speed of mean reversion. 
- The research also investigates the integration of the effect of weather, particularly temperature, into the spark spread option based valuation model. The approach is the first of a kind of research. The study examines the value of the power plant as the operator takes a long position in the temperature-based weather put option and exercises the option in the course of a warmer winter when the spark spread option value drops. Results demonstrate that the expected value of the power plant based on the spark spread option is higher in a cooler winter than that of a warmer winter. However, exercising the weather put option in a warmer winter could add values to the power plant when the expected value from the spark spread option tends to drop. Sensitivity tests with respect to the changes in volatilities and the correlation coefficients are also presented.

- In integrating weather option into the valuation model, pricing of weather option is also important. What price (premium) should be paid to buy or sell a HDD call or put option is a crucial decision. The research examines temperature dynamics for valuing weather option. No one in the literature uses regime-switching models for modeling the temperature dynamics. This thesis examines regime-switching models based on lattice approaches to capture temperature dynamics with an application to the valuation of weather options. The goal of all these approaches is to model the behavior of the expected HDD and CDD in order to calculate the option prices for monthly and seasonal weather contracts.

\subsection{Organization of the thesis}

The rest of the thesis is organized as follows. Chapter 2 discusses the literature on the valuation of power plants. Discretization of the price processes and lattice approaches are presented in Chapter 3. Chapter 4 details the valuation of a natural gas-fired power plant with multiple turbines in presence and absence of carbon allowance price. Integration of spark spread and weather options in valuing a power plant is discussed in Chapter 5. Pricing of weather option is presented in Chapter 6. Chapter 7 summarizes the contributions of this thesis and outlines future work. 


\section{Chapter 2}

\section{Literature Review}

This chapter reviews the existing literature on the valuation approaches and discusses the research gaps. The literature review is more focused on the valuation of gas-fired power plants rather than covering a wide variety of power plants. Natural gas is a cleaner source for electricity generation with lower emissions and lesser impact on the environment in comparison to other fossil fuels like petroleum and coal. The global consumption of natural gas for power generation is expected to increase from $33 \%$ in 2007 to $36 \%$ in 2035 (EIA 2010). Moreover, gas turbine operated power plants offer competitive advantages over other types of power plants, for example, steam turbines, in terms of capital cost, heat rate, operation and maintenance costs, availability and reliability, and time for planning (Boyce 2006).

\subsection{Valuation approaches}

Approaches in valuing power plants can grossly be viewed in two perspectives. One is to view a power plant as a physical asset and the owner has options to operate according to spark spread options. A power plant owner in a deregulated electricity market is free to operate the plant. In the literature, such a power plant is mentioned as a 'merchant' power plant (Wang and Min 2013).

Another way is to consider a power plant from the viewpoint of a financial asset. That is, one may virtually own a power plant as a tolling agreement under which a buyer pays the fuel price and owns the power generated by the plant. Various versions of such agreements may exist. Such a power plant, in the literature, is referred to as a 'synthetic' power plant ( $\mathrm{Li}$ and Kleindorfer 2009).

\subsection{Spark spread option}

A spread is a difference between the prices of two commodities. Spreads are important structures in power plants, refineries, storage facilities, and transmission lines. A call option on spread is similar 
to a call option on futures, except that the underlying security is the spread instead of a futures contract. Common types of spreads are (Eydeland and Wolyniec 2003):

- Quality spread: The price difference between different grades of crude oils, e.g., sweet versus sour crude oil spread.

- Geographic spread: The price difference between two particular locations, e.g., Sonat versus Henry Hub spread.

- Calendar spread: The price difference between different expirations, e.g., June 2008 versus November 2008 futures prices.

- Cross-commodity spread: The price difference between two commodities. For example, crack spread is the price difference between refined products and crude oil, and spark spread is the price difference between electricity and its primary fuel.

The advantage of spread options is that they capture the price uncertainties of both sides (input and output). The valuation of spread options differs from the valuation of other types of options in three ways: (i) instead of a single asset, the behavior of spread options depends on the behavior of two underlying commodities; (ii) the value of the spread option could be negative which is not allowed in other types of options; and (iii) the valuation of a spread depends on the covariance between the price changes of the two commodities (Shimko 1994).

Spark spread is the most useful and prevalent options for gas-fired power plants. It can be expressed as: $\left(P_{e}-H \times P_{n}\right)$, where $P_{e}$ is the price of electricity per MWh, $P_{n}$ is the price of natural gas per MMBtu used to produce the power, and $H$ is the average heat rate in MMBtu/MWh. $H$ is the number of million British Thermal units (MMBtu) required to generate $1 \mathrm{MWh}$ of electricity. The spark spread ties together the price movements of both natural gas and electricity. The payoff of the spark spread option is given by $\max \left(0, P_{e}-H \times P_{n}\right)$. This payoff resembles an option, since the firm has the option of not producing the electricity, when the option does not yield a positive value. A spark spread call option gives the right, not obligation, to generate electricity by burning fuel during the lifetime of the power plant. Considering the operational and maintenance cost, $v$, per MWh of the plant, the spark spread option, $\pi$, can be expressed as:

$$
\pi=\max \left(0, P_{e}-H \times P_{n}-v\right) .
$$




\subsection{The valuation based on spark spread option}

As stated in Section 2.1, for a 'synthetic' power plant, a buyer pays the fuel cost and owns the electricity generated by the power plant. The value of the spark spread option for such a plant is usually priced in the light of Margrabe (1978) analysis of exchange option and the overall value of the plant is calculated as a sum of these individual spark spread options over the valuation period. Studies by Deng et al. (2001), Näsäkkälä and Fleten (2005) and Fleten and Näsäkkälä (2009) fall into this category. While Deng et al. (2001) use separate price processes for electricity and gas, Näsäkkälä and Fleten (2005) model the spark spread itself as a single price process. Deng et al. (2001) propose an analytical method with separate price processes for electricity and natural gas and show that the real options approach provides a plant value closer to the actual market price than that provided by the traditional discounted cash flow (DCF) approach. Two cases are considered: both gas and electricity futures prices follow geometric Brownian motions; both the prices follow mean-reverting processes. Their results show that the geometric Brownian motion, compared to a mean-reverting process assumption, overvalues the spark spread call options. Näsäkkälä and Fleten (2005) assume a two-factor model based on Schwartz and Smith (2000) approach, in which the spark spread is the sum of short-term ( 1 month) and long-term (1 year) forward spark spread prices. The short-term log price is assumed to follow a mean-reverting process and the long-term $\log$ price is assumed to follow an arithmetic Brownian motion. They value both the base load and peak load plant investments. Fleten and Näsäkkälä (2009) use the same methodology to analyze operational flexibilities and the opportunity to abandon the capital equipment in gas-fired power plants.

On the other hand, studies by Gardner and Zhuang (2000), Tseng and Barz (2002), Tseng and Lin (2007), Heydari and Siddiqui (2010), and Wang and Min (2013) use the real options merchant power plant approach. They price the spark spread option by deducting the fuel price directly from the electricity price. A positive spark spread implies that the electricity generation is potentially profitable. Therefore, operating a merchant power plant can be viewed as a series of spark spread options. It also implies operational flexibilities of operating and shutting down the power plant depending on the profitability. Quick start up and shut down characteristics of gas turbines make them suitable for such operations of power plants. Since owning a 'merchant' power plant is like owning a series of spark spread options, the value of a power plant can be estimated as a sum of spark spread options over a planning period (Heydari and Siddiqui 2010; Wang and Min 2013). Gardner and Zhuang (2000) utilize a lattice approach to value a power plant based on spark spread options over a short period of time horizon of five days. They incorporate the minimum on-and-off

times, ramp times, and the minimum dispatch levels for a total of eight different cases. Tseng 
and Lin (2007) propose a lattice framework to value a power plant for two correlated electricity and fuel price processes, in which the correlation coefficient should be less than 0.67. Tseng and Barz (2002) utilizes the Monte Carlo (MC) simulation with a dynamic programming approach for a short-term valuation of a gas-fired power plant. They incorporate variable heat inputs in the cost function and assume both the prices of electricity and gas follow mean-reverting processes. The valuation period is 168 hours (one week). A comparison of spark spread options based valuation under different price processes is studied by Heydari and Siddiqui (2010). Their study uses the UK electricity and natural gas daily spot prices. Regime-switching approaches and mean-reverting approaches with stochastic volatility are compared with the ordinary linear models. They also consider operational flexibility of the plant. Cassano and Sick (2013) analyze optimal operating policies for a power plant based on the market heat rate, which is the ratio of prices between the electricity and the natural gas. The plant is said to be 'out of the money', if the market heat rate is below the plant's operational heat rate. The value of the plant is obtained by multiplying the market heat rate by the natural gas price forward curve. However, environmental regulations on the emission from a power plant pose an impact on the value of a power plant. Moreover, restrictions on $\mathrm{CO}_{2}$ emissions have forced power plant operators to include emission costs in valuing power plants (Heydari and Siddiqui 2010). Table 2.1 summarizes the valuation approaches found in the existing literature.

\subsection{The carbon emission market}

The success of the US Acid Rain program designed to control sulfur dioxide $\left(\mathrm{SO}_{2}\right)$ and nitrogen oxides (NOx) inspired to launch carbon cap-and-trade system. The Acid Rain program is a market based mechanism initiated by the US Environment Protection Agency in order to reduce the causes of acid rain - $\mathrm{SO}_{2}$ and $\mathrm{NOx}$. Carbon cap-and-trade is also a market based mechanism to control carbon emissions. An authority running the cap-and-trade system imposes a cap or limit on the emission and allows firms to trade emission permits among them. It is considered as a promising mechanism to reduce carbon emissions (Carmona and Hinz 2011). In Europe, under a cap-andtrade carbon trading scheme, one emission certificate, also known as European Union Allowance (EUA), allows for emission of one tonne of $\mathrm{CO}_{2}$ (Seifert et al. 2008). Companies can buy or sell such certificates and decide on their amount of $\mathrm{CO}_{2}$ released into the atmosphere. The right to produce a particular amount of $\mathrm{CO}_{2}$ has now become a tradable commodity. A company with lower carbon emissions can benefit from selling its allowances to higher carbon emitting companies (Benz and Trück 2009). According to InterContinentalExchange (ICE) Inc., during the period of from 2007 to 2010, the emission index varied from 8.50 to over 30 Euro per tonne of $\mathrm{CO}_{2}$. Four 
Table 2.1: A review of literature on the valuation of natural gas-fired power plants

\begin{tabular}{|c|c|c|}
\hline \multicolumn{3}{|c|}{ Financial options theory approach: viewing as a synthetic power plant } \\
\hline Author(s) & Objective/Description & Solution approach \\
\hline Hsu (1998) & $\begin{array}{l}\text { Value spark spread options in the spirit } \\
\text { of Black-Scholes type formula on futures contracts. }\end{array}$ & A single period problem \\
\hline Deng et al. (2001) & $\begin{array}{l}\text { Spark spread options are prices } \\
\text { based on Margrabe (1978) type formula. }\end{array}$ & MC simulation \\
\hline Näsäkkälä and Fleten (2005) & $\begin{array}{l}\text { Investment flexibility between base and peak load. } \\
\text { Spark spread is directly modeled to follow the price } \\
\text { process of Schwartz and Smith (2000). }\end{array}$ & Analytical approach \\
\hline Fleten and Näsäkkälä (2009) & Investment analysis of power plant. & Analytical approach \\
\hline \multicolumn{3}{|c|}{ Real options approach: viewing as a merchant power plant } \\
\hline Gardner and Zhuang (2000) & $\begin{array}{l}\text { Spark spread options based short term valuation } \\
\text { with on-and-off constraints. } P_{e} \text { follows MR and } \\
\text { fuel price is assumed constant. }\end{array}$ & $\begin{array}{l}\text { Lattice approach with } \\
\text { backward dynamic } \\
\text { programming }\end{array}$ \\
\hline Tseng and Barz (2002) & $\begin{array}{l}\text { Spark spread options based short term valuation } \\
\text { with operational constraints. }\end{array}$ & MC simulation \\
\hline Tseng and Lin (2007) & Both $P_{e}$ and $P_{n}$ follow MR. & Lattice appraoch \\
\hline Abadie and Chamorro (2008a) & $\begin{array}{l}\text { Valuing investment flexibility either } \\
\text { in a NGCC or an IGCC power plant. }\end{array}$ & Lattice appraoch \\
\hline Heydari and Siddiqui (2010) & $\begin{array}{l}\text { A comparison on spark spread option based } \\
\text { valuation with different processes for } P_{e} \text { and } P_{n} \text {. }\end{array}$ & MC simulation \\
\hline Cassano and Sick (2013) & $\begin{array}{l}\text { Spark spread option based valuation } \\
\text { using the market heat rate. }\end{array}$ & MC simulation \\
\hline Wang and Min (2013) & $\begin{array}{l}\text { Spark spread option based valuation } \\
\text { with forced outage. Both } P_{e} \text { and } P_{n} \text { follow MR. }\end{array}$ & MC simulation \\
\hline Abadie (2015) & $\begin{array}{l}\text { Clean dark and spark spread options based valuation. } \\
P_{e} \text {, fuel and carbon prices are modeled as GBM. }\end{array}$ & MC simulation \\
\hline
\end{tabular}

MC: Monte Carlo simulation; MR: Mean-reverting process; NGCC: Natural gas combined cycle; IGCC: Integrated gasification combined cycle run on coal or gas; GBM: Geometric Brownian motion.

Canadian provinces, Ontario, Quebec, Manitoba, and British Columbia, together with seven U.S. states have established the WCI in order to implement a cap and trade scheme from January, 2012. The initial objective is to reduce emission by $15 \%$ by 2020 from the emission level of 2005 (Mnif and Davison 2011).

Another carbon emission reducing policy is to impose carbon tax. While under the carbon tax policy, the cost of power generation by a power plant is increased by a fixed amount, under the carbon trading system, the cost of generation is varied according to market conditions. Green (2008) finds that a carbon tax policy in lieu of carbon trading would increase the uncertainty in the annual profit of gas-fired power plant by $40 \%$ and would decrease the standard deviation of nuclear power plant's annual profit by almost $40 \%$.

In modeling carbon allowance prices, Seifert et al. (2008) assume that the uncertainty in emission price dynamics is driven by a standard Brownian process. Benz and Trück (2009) analyze the log 
return of carbon spot prices from January, 2005 to December 2006 as a Markov regime-switching model with two regimes (base and spike regimes). While Chesney and Taschini (2012) model the price dynamics as a geometric Brownian motion, Huang (2010) assumes that the process follows either an arithmetic Brownian or a mean reverting motion.

\subsection{The valuation based on clean spark spread option}

Recently stricter environmental rules on $\mathrm{CO}_{2}$ emissions force power plant operators to buy carbon allowances from carbon markets. Power plants covered by an Energy Trading System (ETS) have to consider the cost of carbon dioxide emission allowances (Heydari and Siddiqui 2010). Since 2013, power generators in the most countries in the European Union (EU) must purchase all of their carbon allowances for their carbon emissions (Abadie 2015). The spark spread after deducting the carbon price from it is known as the clean spark spread (CSS). If $P_{c}$ is the cost of carbon emission per ton of $\mathrm{CO}_{2}\left(\mathrm{tCO}_{2}\right)$ and $\mathcal{R}$ is the emission rate in $\mathrm{tCO}_{2} / \mathrm{MWh}$, the CSS options are calculated as: $\max \left(0, P_{e}-H \times P_{n}-\mathcal{R} P_{c}-v\right)$.

In case of a coal power plant, Abadie and Chamorro (2008b) estimate the option value of whether to install a carbon capture and storage facility for the power plant or to purchase carbon allowance. They assume that the carbon prices follow a geometric Brownian motion. Their analysis shows that the investment for the carbon capture and storage is not justified from a financial point of view. Abadie (2015) addresses the valuation of coal-fired and natural gas-fired power plants considering emission costs due to coal and gas, respectively. The price processes are modeled as particular forms of geometric Brownian motion along with seasonalities. Two scenarios are considered and compared. One is to operate the plant without flexibility, that is, irrespective or independent of the value of the spread at each given time; and the another scenario is to operate the plant in a 'cycling' mode with flexibility when the value of the spread is positive.

\subsection{Weather derivatives}

Weather affects daily lives and influences the volume of sales and transactions in everyday trading and business. According to a report of the US Department of Commerce, $70 \%$ of American companies and as much as $22 \%$ of the American gross domestic product (GDP) are sensitive to weather (Ku 2001). The snowfalls in the UK in December 2010 caused a loss of millions to the British Airports Authority (BAA), the operator of five British airports including Heathrow airport, when it was closed for four days (Thornton 2011). The warm weather during the winter of 2006 in the northeastern part of the USA prompted a drastic decline in the demand for winter clothes, such 
as sweaters and coats (Chen and Yano 2010). These examples are a few among many instances of adverse weather effects that continuously pose huge financial risks to all kinds of businesses. To hedge such weather related financial risks, a financial instrument called weather derivative is introduced in the financial market in the form of: futures, options on futures or swaps, based on underlying weather variables, such as temperature, rainfall, and snowfall. Through weather derivatives, a business organization can transfer its weather related risks to someone, who is willing to absorb the risk, contingent on a predetermined value or index of the underlying weather variables. According to a Weather Risk Management Association (WRMA 2003) report, more than 90\% of weather derivatives are temperature-based. Degree-days indices, e.g., heating degree days (HDD) or cooling degree days (CDD), are considered as the underlying assets of the temperature-based weather derivatives.

Weather derivatives differ from standard financial derivatives in several aspects. Firstly, the underlying asset (e.g., Heating degree days (HDD) or Cooling degree days (CDD)) is not tradable in the spot market. Secondly, standard financial derivatives hedge the price risk, while weather derivatives hedge the volume or quantity risk. Thirdly, weather is a location-specific and nonstandardized commodity, unlike a standardized commodity, such as a particular light sweet crude oil (e.g., Campbell and Diebold 2005; London 2007). Weather derivatives also differ from insurance (Alaton et al. 2002). Payout of weather derivatives is based only on the actual outcome of the weather (e.g., HDD or CDD), not on damages and losses due to weather. The holder of a weather derivative, unlike the holder of an insurance contract, does not have to file a claim and prove damages to receive a payout. While weather derivatives cover low-risk, high-probability events (e.g., uncertainties in weather), insurance protects high-risk, low-probability events (e.g., earthquake, flood, etc.) (Cao and Wei 2004).

A standard weather derivative contract usually includes six features: (i) a contract period, for example, a monthly contract for the month of December; (ii) a measurement station, for instance, Pearson International Airport, Toronto, Canada; (iii) a weather variable, for example, temperature, measured at the measurement station over the contract period; (iv) an index, for instance, HDD or CDD, which aggregates the weather variable over the contract period; (v) a pay-off function that converts the index into cash flow; and (vi) a premium or option price paid by the buyer at the start of the contract (Jewson and Brix 2005).

The Chicago Mercantile Exchange (CME) introduced electronic trading of weather derivatives in September 1999 (London 2007). The CME offers trading with futures contracts as well as options on futures based on the degree days index. The CME rulebook chapter 421 (rule 42101) states that "each particular CME Canadian degree days index is the accumulation of like degree days over a calender month. The accumulation period of each CME-HDD or CME-CDD index futures contract 
begins with the first calender day of the contract month and ends with the last calender day of the contract month." The HDD contract months for Canadian degree days index options at CME are from January to April inclusive as well as from October to December inclusive, and the CDD contract months are from April to October inclusive. April and October are called shoulder months and are included as HDD and CDD months. The options on futures are of European style, as they can only be exercised at the expiration date (Alaton et al. 2002). At CME, there are both futures and options contracts on temperature-based derivatives for cities as follows: six Canadian (Calgary, Edmonton, Montreal, Toronto, Vancouver, and Winnipeg), twenty-four US, ten European, three Australian, and two Asian.

Degree day (DD) indices originated in the energy industry and are designed to correlate with the domestic demand of heating and cooling (Jewson and Brix 2005). HDD are used to measure the demand for heating and are thus a measure of how cold it is. The HDD index over the period from day 1 to day $N$ is defined as: $\operatorname{HDD}=\sum_{t=1}^{N} \max \left\{\bar{Q}-Q_{t}, 0\right\}$, where $\bar{Q}=$ Baseline temperature (usually $65^{0} \mathrm{~F}$ in USA, $18^{0} \mathrm{C}$ in other countries ${ }^{1}$ ) and $Q_{t}=\frac{Q_{t, \max }+Q_{t, \min }}{2}$, where $Q_{t, \max }$ and $Q_{t, \min }$ are the maximum and minimum temperatures on day $t$, respectively. Cooling degree days (CDD), on the other hand, are used to measure the demand for cooling. The hotter the day, the more CDD. The CDD index over the period from day 1 to day $N$ is: $\operatorname{CDD}=\sum_{t=1}^{N} \max \left\{Q_{t}-\bar{Q}, 0\right\}$.

The first weather derivative transaction was executed in 1997 by an energy provider, Aquila Energy (Considine 1999). An electric service provider, Consolidated Edison Company (ConEd) in the city of New York, USA, purchased electric power from Aquila Energy for the month of August at an agreed price, along with a weather clause that Aquila would pay ConEd a rebate, if the month of August turned out to be cooler than expected. In 2007, Weatherproof, a clothing manufacturer in the US, purchased a weather derivative brokered by StormExchange, a weather-related financial hedging firm, contingent on whether the month of December would be warmer than usual (Chen and Yano 2010). Similarly, a power generation company, GenCo, could hedge its weather risks by taking a long position on a HDD put option for a winter season, for example, from January to March. If the winter is very cold, electricity demand will go up and GenCo receives high revenues. On the other hand, if the winter is not so cold and HDD falls below a strike HDD, the company exercises the option and could compensate the downward revenue. In either situation, GenCo could reduce its exposure to weather risk. The crucial decision here is to figure out the appropriate option price (premium), i.e., what price should be paid to buy or sell a HDD option. Consequently, it greatly depends on the appropriate modeling of the temperature dynamics. This thesis presents regime-switching models to capture the behavior of temperature dynamics with an aim to the

\footnotetext{
${ }^{1}$ Usually the base temperature of $18^{\circ} \mathrm{C}$ is a temperature below which people tend to consume more energy to heat their residences and above which people start to turn on their air conditioners for cooling (Alaton et al. 2002).
} 
valuation of weather option price.

Different forms of Ornstein-Uhlenbeck mean-reverting models to capture the dynamics of temperature are dominant in the literature for pricing weather options. For example, the stochastic component of daily temperature, $w$, follows a mean-reverting process $d w=\kappa_{w}(\bar{w}-w) d t+\sigma_{w} d B_{w}$, where $\kappa_{w}$ is the speed of the mean reversion, $\bar{w}$ is the long-term mean of the process, $\sigma_{w}$ is the volatility of the process, and $d B_{w}$ is the increment of the Wiener process over an infinitesimally small period of time such that $\mathbf{E}\left(d B_{w}\right)=0$ and $\operatorname{Var}\left(d B_{w}\right)=d t$. Dornier and Queruel (2000) first began investigation on the futures prices based on temperature indices. They model the temperature observations at O'Hare airport in Chicago, USA, as an Ornstein-Uhlenbeck mean-reverting process with constant $\sigma$. Alaton et al. (2002) extend the Ornstein-Uhlenbeck process with monthly variations in $\sigma$ to fit the temperature observations at the Stockholm-Bromma airport, Sweden. Brody et al. (2002) propose a different modeling approach of the Ornstein-Uhlenbeck temperature model, assuming $d z$ is the increment of fractional Brownian motion, instead of the Wiener process, with constant $\sigma$. They use the temperature of the city of London, UK, and calculate prices for different temperature derivatives. Benth and Šaltytė-Benth (2005) propose an Ornstein-Uhlenbeck process driven by a generalized hyperbolic Lèvy process for Norwegian temperature data.

Campbell and Diebold (2005) use an autoregressive moving average (ARMA) time series model for temperature and observe seasonality in the autocorrelation function for the (squared) residuals when modeling the temperature in US cities. They propose a seasonal autoregressive conditional heteroskedastic $(\mathrm{ARCH})$ process to model $\sigma$. Benth and Šaltytė-Benth (2007) propose an OrnsteinUhlenbeck process with seasonal volatility to model the dynamics of the temperature data from Stockholm, Sweden. Benth et al. (2007) propose a continuous-time autoregressive (CAR) process to model Swedish temperature data. Davis (2001) suggests a stochastic discount factor (SDF) approach (an approach of mathematical economics) to price weather options by modeling the accumulated HDD as a log-normal process. Cao and Wei (2004) propose an equilibrium pricing model based on the fact that, in equilibrium, both the financial market and the goods market are cleared so that the aggregate consumption equals the dividend generated from the risky stock. They calculate SDF, and use this factor to price weather derivatives. These weather derivatives pricing approaches are summarized in Table 2.2. 
Table 2.2: Summary of approaches to pricing weather derivatives

\begin{tabular}{l|l|l}
\hline Authors & Model & Dataset \\
\hline \multicolumn{2}{c}{ Stochastic modeling approaches } \\
\hline $\begin{array}{l}\text { Dornier and Queruel (2000) } \\
\text { Alaton } \text { et al. }(2002)\end{array}$ & Ornstein-Uhlenbeck Model & Chicago, USA \\
Brody et al. $(2002)$ & Ornstein-Uhlenbeck Model & Stockholm, Sweden \\
\multicolumn{2}{c}{ Time series approaches } & London, UK \\
\hline Campbell and Diebold (2005) & AutoRegressive Moving Average (ARMA) & US cities \\
Benth et al. (2007) & Continuous time AutoRegression (CAR) & Stockholm, Sweden \\
\hline \multicolumn{2}{c}{ Mathematical economics approaches } \\
\hline $\begin{array}{l}\text { Davis (2001) } \\
\text { Cao and Wei (2004) }\end{array}$ & Stochastic Discount Factor (SDF) & London, UK \\
\hline
\end{tabular}

\subsection{Research gaps}

Valuation models of natural gas-fired power plants in the literature are based mostly on spark spread option. Under environmental restrictions, carbon allowance price plays an important role. Moreover, a power plant can possess one or multiple generators. In the US, there are about 7,000 power plants having around 19,000 individual generators (EIA 2015). While studies in the literature consider power plants with a single turbine operating at a constant heat rate, this thesis examines the valuation of a natural gas-fired power plant with multiple turbines, each of them having different power generation capacities, input-output characteristics and carbon emission rates.

This thesis also investigates the effect of flexibility of switching from one turbine to another based on price movements in absence and presence of carbon allowance price. In the existing literature, for example, in Heydari and Siddiqui (2010) and Abadie (2015), the operational flexibility is considered as operating the single turbine in 'cycling mode' of 'on' and 'off' in accordance with the spark spread option against the scenario that the turbine is operated irrespective of the spark spread option value. In contrast, the thesis investigates the valuation of a merchant power plant based on the spark and clean spark spread options with and without utilizing operational flexibility among multiple gas turbines. That is, the power plant is assumed to have multiple gas turbines each of which is in cycling mode in accordance with price movements at each given time. The operational flexibility is, therefore, obtained from switching the power plant state, which is achieved by a combination of operating turbines.

While the existing literature focuses on the valuation based on only spark spread options, this research study incorporates the environment temperature effect on the power plant valuation. In an usual cold winter, the payoff from the spark spread option is expected to be higher, while in a warmer winter the payoff from the spark spread option is expected to go downward. To add values to this downside payoff in a warmer winter, the plant owner can opt to hold a long position in a temperature-based weather put option. This thesis investigates the integration of spark spread and 
weather options in the valuation model of a power plant and to the best of the author's knowledge, the approach is the first of a kind of approach. One of the objectives of the research is, therefore, to investigate the valuation of a power plant integrating spark spread and weather options for a natural gas-fired power plant run by multiple gas turbines.

Incorporating weather option into spark spread based option valuation model requires the pricing of weather option itself. Modeling the temperature dynamics is essential for pricing temperaturebased weather options. This thesis also examines temperature dynamics with an aim to pricing temperature-based weather option. Huisman (2008) demonstrates that a shift in mean temperature induces regime-switching behavior in electricity prices and increases the probability of spike occurrences. Therefore, there lies clues that temperature may also show regime-switching behavior. 


\section{Chapter 3}

\section{Methodology}

The thesis is focused on valuation models. Assessing the value of a power plant entails modeling the correlated movements of price processes. The sequence of identically distributed random variables is known as a process or alternatively a stochastic process. Capturing the behavior of the price processes is an essential aspect of the valuation models. Since discrete-time lattice framework is convenient for valuation models, the processes are then discretized via nodes and branches as a lattice by matching the moments of the corresponding continuous-time processes. After having the discretized values at each node, possible states and actions are decided based on an optimization model. A recursive solution approach moving backward from the last layer is then applied by merging discounted subsequent values into the current decision. The overall scheme of the thesis is depicted in Figure 3.1. 


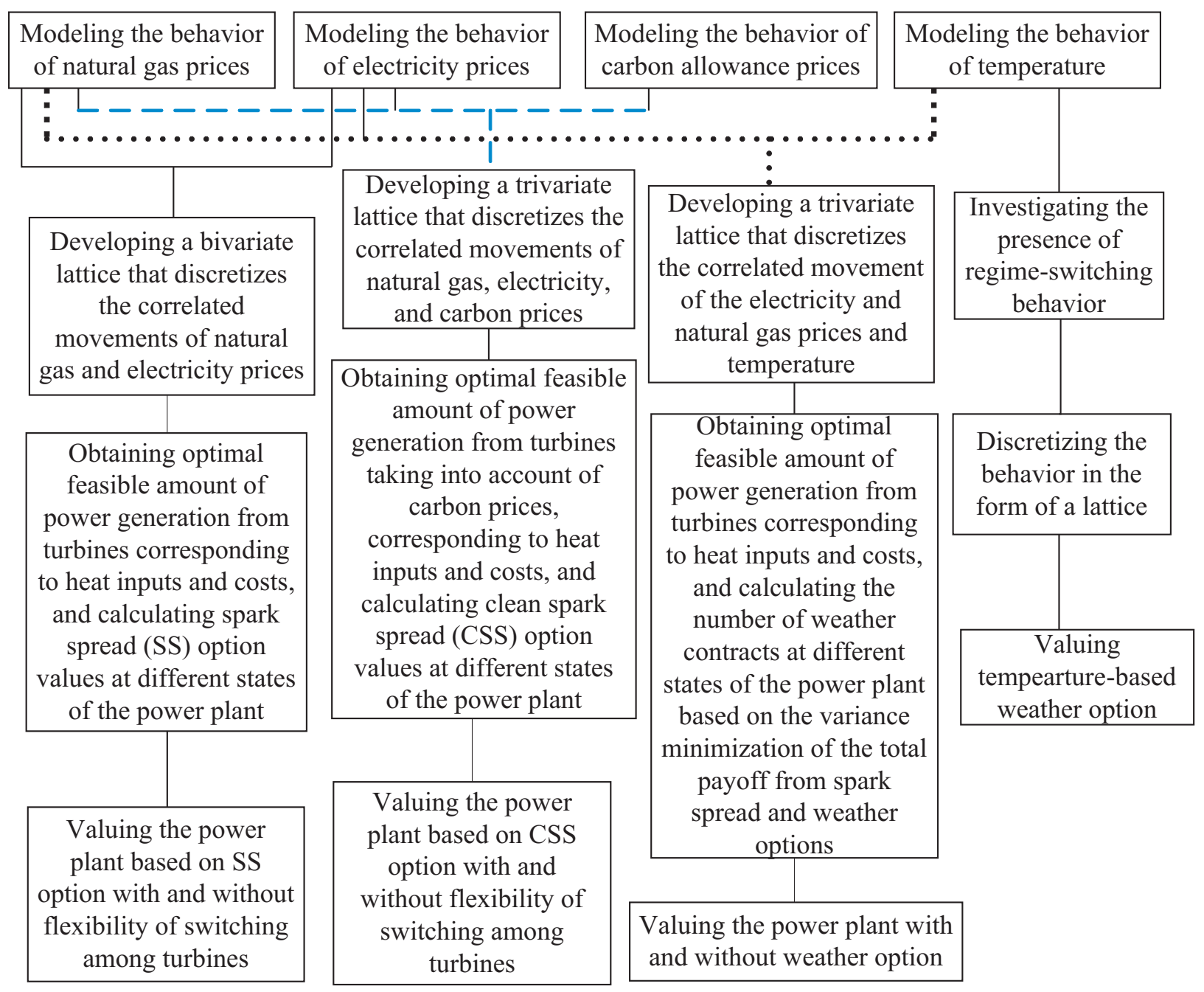

Figure 3.1: The overall scheme of the thesis. 


\subsection{Contingent claim valuation approach}

The traditional valuation approach values an utility or a project by discounting the expected cash flows at the project's cost of capital, which is estimated by the capital asset pricing model (CAPM). The CAPM approach states that an expected return from an asset requires a risk premium over the risk-free return for the systematic, market or nondiversifiable risk (Trigeorgis 1996). The systematic or nondiversifiable risk (e.g., economy wide forces affecting the market, the budget deficit or inflation) is represented by a coefficient named 'beta', which is measured by the covariance between the asset return and the market return (e.g., the S\&P 500 stock index) over the variance of the market return. However, determining the exact value of the 'beta' may be difficult due to its dependence on the pattern of the expected cash flows over time, the growth rate of the expected cash flows, and the procedure by which the investors revise the cash flows (Trigeorgis 1996).

The financial option pricing models use the risk-neutral valuation approach, in which a portfolio, for example, buying a number of shares of an underlying security and borrowing an appropriate amount of money at the riskless rate, can be constructed that exactly replicates the option payoff in any state of the world (Trigeorgis 1996; Hull 2002). Therefore, the ability of constructing a portfolio cancels out the risk and helps to obtain the value of an option in a world where an investor's risk attitude is irrelevant. In such a risk-neutral world, all assets would earn the risk-free return and the expected cash flow can be discounted at the risk-free rate (Trigeorgis 1996).

In case of the real-option valuation, the underlying asset often cannot be traded, for example, an R\&D project or temperature. However, whether traded or non traded, any contingent claim on an asset can be valued by replacing the expected return with a certainty-equivalent return that enables one to assume that, as if, the world were risk neutral (Trigeorgis 1996). The certaintyequivalent return, in other words, the risk-adjusted expected return, is obtained by deducting the risk premium (the excess return over the risk-free return) from the process drift. The risk premium is measured by the market price of risk for the asset multiplied by its volatility (Hull 2002). The market price of risk is a reward to risk ratio, that is, the excess return above the risk-free rate per unit of risk. "If the market price of risk (e.g., for an R\&D project or for temperature) is zero, investor's would be neutral to the asset's risk" (Trigeorgis 1996). Futures prices are the expected value of the future spot prices in a risk-neutral world, in other words, the futures prices are the certainty-equivalent of spot prices. Therefore, the valuation of an asset using futures prices can be obtained by discounting at the risk-free rate (Abadie et al. 2013). 


\section{$3.2 \quad$ Price processes}

Commodity prices, such as the prices of oil, copper, sugar and natural gas, usually follow meanreverting processes. The price may fluctuate randomly up and down in the short run, but in the long run it moves towards the mean price value. The reason for such behavior is explained from the microeconomic point of view (Dixit and Pindyck 1994). When the price of a product moves below the long-term mean price, demand for this product increases with diminishing production. This causes the price to move towards the mean price. The opposite will happen if the price goes up above the long-term mean value. Prices of electricity and natural gas are assumed to follow mean-reverting processes (see Appendix A for details). An argumentive discussion on it has been provided by Abadie and Chamorro (2008a).

Let the price of natural gas at day $t$ be $P_{n t}$. The logarithm of natural gas price, $x_{t}=\log \left(P_{n t}\right)$, can be expressed as a mean-reverting process, also known as Ornstein-Uhlenbeck process:

$$
d x_{t}=\kappa_{x}\left(\bar{x}-x_{t}\right) d t+\sigma_{x} d B_{x}
$$

where $\kappa_{x}$ is the mean-reverting coefficient or speed of reversion, $\bar{x}$ is the long-term mean price, $\sigma_{x}$ is the volatility of the process, and $d B_{x}$ is the increment of the Wiener process, a normally distributed random increment with a mean of 0 and a variance of $d t$.

Similarly, if the price of electricity at day $t$ be $P_{e t}$, then its logarithmic value, $y_{t}=\log P_{e t}$, is assumed to follow a mean-reverting process and can be written as:

$$
d y_{t}=\kappa_{y}\left(\bar{y}-y_{t}\right) d t+\sigma_{y} d B_{y}
$$

where $\kappa_{y}$ is the mean-reverting coefficient, $\sigma_{y}$ is the volatility of the process, $\bar{y}$ is the long-term mean price, and $d B_{y}$ is the increment of the Wiener process.

In the same fashion, the logarithm of carbon allowance price at day $t, z_{t}=\log P_{c t}$, is assumed to follow a mean-reverting process and can be written as:

$$
d z_{t}=\kappa_{z}\left(\bar{z}-z_{t}\right) d t+\sigma_{z} d B_{z}
$$

where $\bar{z}$ is its long-term mean, $\kappa_{z}$ is the the speed of reversion, $\sigma_{z}$ is the process volatility, and $d B_{z}$ is the increment of the Wiener process.

The relationships among the increments of the processes are: between the prices of electricity and natural gas, $d B_{x} d B_{y}=\rho_{x y} d t$; between the prices of electricity and carbon, $d B_{y} d B_{z}=\rho_{y z} d t$; and between the prices of carbon and natural gas, $d B_{z} d B_{x}=\rho_{z x} d t$, where correlations, $\rho_{x y}, \rho_{y z}$, 
and $\rho_{z x}$, represent the degree to which the increments move in the same or opposite directions.

Similar to the prices of natural gas and electricity, the daily mean temperature also exhibits the mean-reverting behavior (Dornier and Queruel 2000; Alaton et al. 2002). Dornier and Queruel (2000) model temperature observations at the O'Hare airport in Chicago, USA, as an OrnsteinUhlenbeck mean-reverting process with constant volatility. Alaton et al. (2002) extend this process with monthly variations in volatilities to fit the temperature observations at the Stockholm-Bromma airport, Sweden. The mean temperature is calculated as the daily average of the maximum and minimum temperature. On day $t$, the daily mean temperature $Q_{t}$ can be modeled as: $Q_{t}=w_{t}+S_{t}$, where $S_{t}$ is the deterministic annual seasonality component and $w_{t}$ is the deseasonalized stochastic component. After removing the seasonality component from temperature data, the stochastic component of temperature data, $w_{t}=Q_{t}-S_{t}$, is analyzed for the valuation model. For the spark spread and weather options based valuation model, a trivariate lattice is constructed taking the logarithm of electricity and natural gas prices and the stochastic component of temperature, along with corresponding step sizes and branch probabilities. The prices are then retrieved by taking exponentials and the temperature is retrieved by adding the seasonal component to the exponential of the stochastic component. Details of the lattice model is discussed in the following section.

\subsection{Lattice representation of price processes}

Modeling correlated behavior of more than one stochastic variable is a complex task. It is also very hard or almost impossible to derive an analytical closed-form solution for such a model (Hull 2002). Therefore, a numerical method, such as the lattice approach or Monte Carlo simulation, is employed to represent the stochastic behavior of prices. However, the Monte Carlo simulation is computationally intensive as it repeatedly imitates numerous scenarios of prices following the prescribed stochastic processes. For example, the valuation of an "American option with a sixweek maturity, the lattice approach (coded in C language) takes around 13.5 seconds, whereas the Monte Carlo simulation (coded in MATLAB) takes around 10,000 seconds" (Wahab and Lee 2009). The lattice approach, on the other hand, represents the predicted values of a stochastic variable in a more intuitive way. The discrete-time framework of the lattice approach is also suitable and convenient for a real options valuation (Hahn and Dyer 2008).

Stochastic price movements in discrete times can be represented by a lattice. Cox et al. (1979) model a single continuous-time geometric Brownian motion (GBM) into a binomial lattice in order to value option prices. Boyle (1988) develops lattice models for valuing option prices for multiple underlying assets following GBM. Nelson and Ramaswamy (1990) present a binomial lattice to 
represent mean-reverting processes. Hahn and Dyer (2008) model two correlated mean-reverting processes utilizing Nelson and Ramaswamy (1990) approach along with the application of Bayes' probability law. In the same spirit, Abadie et al. (2013) develop a lattice model for three correlated stochastic processes.

The strength of the lattice approach over the other non-lattice approaches (e.g., the Monte Carlo simulation and the finite difference method) lies in the fact that: (i) a lattice represents the possible future values of a regime-switching variable in a more intuitive way; (ii) the discrete-time framework of a lattice allows the valuation of European-style weather options more conveniently; (iii) a lattice approach is flexible enough to incorporate branch probabilities that change over time; and (iv) it is easy to implement and use (Bollen 1998; Hull 2002; Wahab and Lee 2009).

\subsubsection{A bivariate lattice for spark spread option}

Hahn and Dyer (2008) extend Nelson and Ramaswamy (1990) approach to develop a bivariate lattice representing the movement of two correlated mean-reverting processes. Let the processes in Equation (3.1) and Equation (3.2) have a relationship with a correlation $\rho_{x y} d t=d B_{x} d B_{y}$. A bivariate binomial lattice can be developed for this joint process such that one node generates four nodes at each discrete interval (Clewlow and Strickland 1998). For example, if the initial node has a coordinate $(x, y)$, then the coordinates of the four nodes at the next time-step are: $\left(x+\Delta_{x}, y+\Delta_{y}\right)$, $\left(x-\Delta_{x}, y+\Delta_{y}\right),\left(x+\Delta_{x}, y-\Delta_{y}\right)$, and $\left(x-\Delta_{x}, y-\Delta_{y}\right)$, representing up-up, down-up, up-down, and down-down movements, respectively (see Figure 3.2).
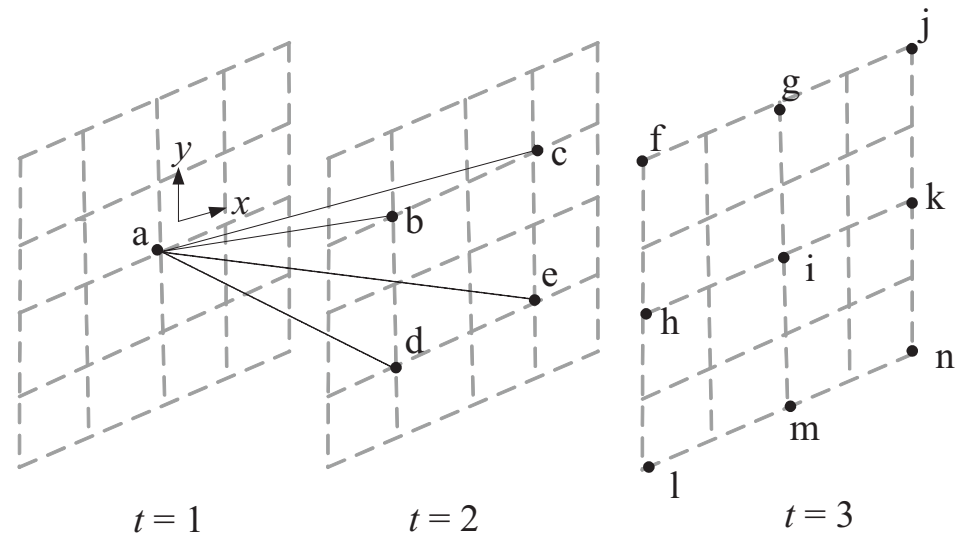

Figure 3.2: A lattice representing correlated movement of two variables. 
To define the joint probabilities, the step size can be set to:

$$
\begin{aligned}
& \Delta_{x}=\sigma_{x} \sqrt{\Delta t} \\
& \Delta_{y}=\sigma_{y} \sqrt{\Delta t}
\end{aligned}
$$

Since two recombining lattices are merged together, the resulting bivariate lattice is, therefore, also recombining. The probabilities of four possible correlated movements (up-up, down-up, up-down, and down-down) of $x$ and $y$ can be determined by matching the mean and variance of a two-variable binomial process (see also Appendix B) :

$$
\begin{aligned}
& p_{u u}=\frac{\Delta_{x} \Delta_{y}+\Delta_{y} \nu_{x} \Delta t+\Delta_{x} \nu_{y} \Delta t+\rho_{x y} \sigma_{x} \sigma_{y} \Delta t}{4 \Delta_{x} \Delta_{y}}, \\
& p_{u d}=\frac{\Delta_{x} \Delta_{y}+\Delta_{y} \nu_{y} \Delta t-\Delta_{x} \nu_{y} \Delta t-\rho_{x y} \sigma_{x} \sigma_{y} \Delta t}{4 \Delta_{x} \Delta_{y}}, \\
& p_{d u}=\frac{\Delta_{x} \Delta_{y}-\Delta_{y} \nu_{y} \Delta t+\Delta_{x} \nu_{y} \Delta t-\rho_{x y} \sigma_{x} \sigma_{y} \Delta t}{4 \Delta_{x} \Delta_{y}}, \\
& p_{d d}=\frac{\Delta_{x} \Delta_{y}-\Delta_{y} \nu_{y} \Delta t-\Delta_{x} \nu_{y} \Delta t+\rho_{x y} \sigma_{x} \sigma_{y} \Delta t}{4 \Delta_{x} \Delta_{y}},
\end{aligned}
$$

where $\nu_{x}$ and $\nu_{y}$ are the drifts of the respective processes and are given by: $\nu_{x}=\kappa_{x}\left(\bar{x}-x_{t}\right)-(1 / 2) \sigma_{x}^{2}$ and $\nu_{y}=\kappa_{y}\left(\bar{y}-y_{t}\right)-(1 / 2) \sigma_{y}^{2}$, respectively (Hahn and Dyer 2008). The marginal probabilities of $x$ are given by,

$$
\begin{aligned}
& p_{u}=\frac{1}{2}+\frac{1}{2} \frac{\nu_{x} \Delta t}{\Delta_{x}} \\
& p_{d}=\frac{1}{2}-\frac{1}{2} \frac{\nu_{x} \Delta t}{\Delta_{x}}
\end{aligned}
$$

Using Bayes' rule of probabilities, the joint process can be expressed as the product of the marginal binomial process for $x$ and the conditional binomial process for $y$ as: $p(x, y)=p(y \mid x) p(x)$. The conditional probabilities are:

$$
\begin{aligned}
& p_{u \mid u}=\frac{\Delta_{x}\left(\Delta_{y}+\Delta t \nu_{y}\right)+\Delta t\left(\Delta_{y} \nu_{x}+\rho_{x y} \sigma_{x} \sigma_{y}\right)}{2 \Delta_{y}\left(\Delta_{x}+\Delta t \nu_{x}\right)}, \\
& p_{d \mid u}=\frac{\Delta_{x}\left(\Delta_{y}-\Delta t \nu_{y}\right)+\Delta t\left(\Delta_{y} \nu_{x}-\rho_{x y} \sigma_{x} \sigma_{y}\right)}{2 \Delta_{y}\left(\Delta_{x}+\Delta t \nu_{x}\right)}, \\
& p_{u \mid d}=\frac{\Delta_{x}\left(\Delta_{y}-\Delta t \nu_{y}\right)+\Delta t\left(\rho_{x y} \sigma_{x} \sigma_{y}-\Delta_{y} \nu_{x}\right)}{2 \Delta_{y}\left(\Delta_{x}+\Delta t \nu_{x}\right)} . \\
& p_{d \mid d}=\frac{\Delta_{x}\left(\Delta_{y}+\Delta t \nu_{y}\right)-\Delta t\left(\Delta_{y} \nu_{x}+\rho_{x y} \sigma_{x} \sigma_{y}\right)}{2 \Delta_{y}\left(\Delta_{x}+\Delta t \nu_{x}\right)},
\end{aligned}
$$

Since the model is based on Nelson and Ramaswamy (1990) general approximation, it is necessary to censor probabilities when the amount of mean-reversion causes probabilities greater than one or 
less than zero. That is,

$$
\begin{aligned}
& p_{u}^{*}=\max \left(0, \min \left(1, p_{u}\right)\right) \\
& p_{d}^{*}=1-p_{u}^{*},
\end{aligned}
$$

where the star symbol $(*)$ represents censoring probabilities in between 0 and 1 . Therefore, the branch probabilities can be expressed as:

$$
\begin{aligned}
p_{u u}^{*} & =p_{u}^{*} \cdot p_{u \mid u}^{*} \\
p_{u d}^{*} & =p_{u}^{*} \cdot p_{d \mid u}^{*} \\
p_{d u}^{*} & =p_{d}^{*} \cdot p_{u \mid d}^{*} \\
p_{d d}^{*} & =p_{d}^{*} \cdot p_{d \mid d}^{*}
\end{aligned}
$$

\subsubsection{A trivariate lattice for clean spark spread option}

A trivariate lattice can be built to represent movements of three correlated variables - natural gas, electricity and carbon allowance prices, each of which is assumed to follow a mean-reverting process. In addition to the increments for stochastic movements of natural gas and electricity prices defined in Equations (3.4)-(3.5), the step size for carbon allowance price can be expressed as:

$$
\Delta_{z}=\sigma_{z} \sqrt{\Delta t}
$$

Figure 3.3 shows the movement of three variables in the form of a cube. With three variables, each node of the lattice generates $2^{3}=8$ nodes in the next layer, for example, $\left(x+\Delta_{x}, y+\Delta_{y}, z+\Delta_{z}\right)$, $\left(x+\Delta_{x}, y-\Delta_{y}, z-\Delta_{z}\right)$, and $\left(x-\Delta_{x}, y+\Delta_{y}, z-\Delta_{z}\right)$. Details can be found in Appendix C. Branch

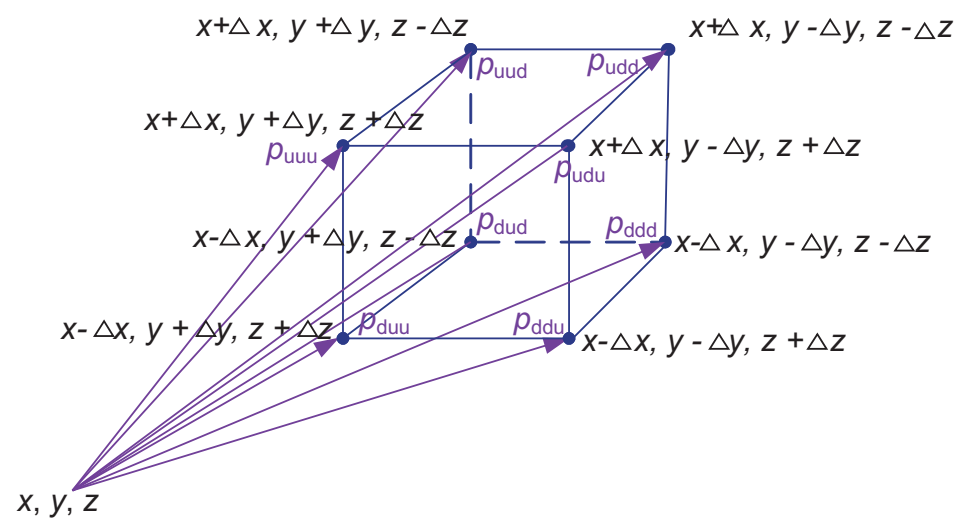

Figure 3.3: A lattice representing correlated movement of three variables. 
probabilities are calculated based on the method suggested by Boyle et al. (1989) for multivariate contingent claims. These probabilities can be expressed as:

$$
\begin{aligned}
& p_{\text {uuu }}=\frac{1}{8}\left[1+\rho_{x y}+\rho_{y z}+\rho_{z x}+\sqrt{\Delta t}\left(\frac{\nu_{x}}{\sigma_{x}}+\frac{\nu_{y}}{\sigma_{y}}+\frac{\nu_{z}}{\sigma_{z}}\right)\right] \\
& p_{\text {uud }}=\frac{1}{8}\left[1+\rho_{x y}-\rho_{y z}-\rho_{z x}+\sqrt{\Delta t}\left(\frac{\nu_{x}}{\sigma_{x}}+\frac{\nu_{y}}{\sigma_{y}}-\frac{\nu_{z}}{\sigma_{z}}\right)\right] \\
& p_{u d u}=\frac{1}{8}\left[1-\rho_{x y}+\rho_{y z}-\rho_{z x}+\sqrt{\Delta t}\left(\frac{\nu_{x}}{\sigma_{x}}-\frac{\nu_{y}}{\sigma_{y}}+\frac{\nu_{z}}{\sigma_{z}}\right)\right] \\
& p_{\text {udd }}=\frac{1}{8}\left[1-\rho_{x y}-\rho_{y z}+\rho_{z x}+\sqrt{\Delta t}\left(\frac{\nu_{x}}{\sigma_{x}}-\frac{\nu_{y}}{\sigma_{y}}-\frac{\nu_{z}}{\sigma_{z}}\right)\right] \\
& p_{d u u}=\frac{1}{8}\left[1-\rho_{x y}-\rho_{y z}+\rho_{z x}+\sqrt{\Delta t}\left(-\frac{\nu_{x}}{\sigma_{x}}+\frac{\nu_{y}}{\sigma_{y}}+\frac{\nu_{z}}{\sigma_{z}}\right)\right] \\
& p_{d u d}=\frac{1}{8}\left[1-\rho_{x y}+\rho_{y z}-\rho_{z x}+\sqrt{\Delta t}\left(-\frac{\nu_{x}}{\sigma_{x}}+\frac{\nu_{y}}{\sigma_{y}}-\frac{\nu_{z}}{\sigma_{z}}\right)\right] \\
& p_{d d u}=\frac{1}{8}\left[1+\rho_{x y}-\rho_{y z}-\rho_{z x}+\sqrt{\Delta t}\left(-\frac{\nu_{x}}{\sigma_{x}}-\frac{\nu_{y}}{\sigma_{y}}+\frac{\nu_{z}}{\sigma_{z}}\right)\right] \\
& p_{d d d}=\frac{1}{8}\left[1+\rho_{x y}+\rho_{y z}+\rho_{z x}+\sqrt{\Delta t}\left(-\frac{\nu_{x}}{\sigma_{x}}-\frac{\nu_{y}}{\sigma_{y}}-\frac{\nu_{z}}{\sigma_{z}}\right)\right],
\end{aligned}
$$

where, $\nu_{x}=\kappa_{x}\left(\bar{x}-x_{t}\right)-(1 / 2) \sigma_{x}^{2}, \nu_{y}=\kappa_{y}\left(\bar{y}-y_{t}\right)-(1 / 2) \sigma_{y}^{2}$, and $\nu_{z}=\kappa_{z}\left(\bar{z}-z_{t}\right)-(1 / 2) \sigma_{z}^{2}$, respectively (Hahn and Dyer 2008). In case of a mean-reverting $\mathrm{CO}_{2}$ process, $\nu_{z}=\kappa_{z}\left(\bar{z}-z_{t}\right)-(1 / 2) \sigma_{z}^{2}$ and in case of a geometric Brownian motion modeling of $\mathrm{CO}_{2}$ prices, $\nu_{z}=\mu_{z}-(1 / 2) \sigma_{z}^{2}$. Since probability cannot be negative, applying the similar method employed by Hahn and Dyer (2008) and Abadie et al. (2013), the obtained branch probabilities are:

$$
\begin{aligned}
& p_{u u u}^{*}=p_{u}^{*} \cdot p_{u / u}^{*} \cdot p_{u / u / u}^{*} \\
& p_{u u d}^{*}=p_{u}^{*} \cdot p_{u / u}^{*} \cdot p_{d / u / u}^{*} \\
& p_{u d u}^{*}=p_{u}^{*} \cdot p_{d / u}^{*} \cdot p_{u / d / u}^{*} \\
& p_{u d d}^{*}=p_{u}^{*} \cdot p_{d / u}^{*} \cdot p_{d / d / u}^{*} \\
& p_{d u u}^{*}=p_{d}^{*} \cdot p_{u / d}^{*} \cdot p_{u / u / d}^{*} \\
& p_{d u d}^{*}=p_{d}^{*} \cdot p_{u / d}^{*} \cdot p_{d / u / d}^{*} \\
& p_{d d u}^{*}=p_{d}^{*} \cdot p_{d / d}^{*} \cdot p_{u / d / d}^{*} \\
& p_{d d d}^{*}=p_{d}^{*} \cdot p_{d / d}^{*} \cdot p_{d / d / d}^{*}
\end{aligned}
$$

\subsection{Regime--switching models}

The concept behind the Markov regime-switching model is to capture the observed stochastic behavior of an underlying variable by more than one regime. For each regime, one can define an 
independent underlying process (Hamilton 2008). The switching behavior between the regimes (states) is assumed to be governed by an unobservable latent variable $R_{t}$. For example, $\mathbb{L}_{t}=\{1,2\}$ in a two-state regime-switching model. A two-state Markov chain governs the description of the probability law of switching between $\mathbb{L}_{t}=1$ and $\mathbb{L}_{t}=2$ with transition probabilities

$$
\gamma_{i j}=\operatorname{Pr}\left(\mathbb{L}_{t}=j \mid \mathbb{L}_{t-1}=i\right) \text { for } i, j=1,2 .
$$

Transition probability is defined as the probability of switching between regimes from one observation to the next observation.

There are many applications of regime-switching models in the literature. Bollen et al. (2000) use regime-switching models to capture the dynamics of foreign exchange rates. Andrew and Bekaert (2002) examine the econometric performance of regime-switching models for interest rates of the USA, the UK, and Germany. Huisman and Mahieu (2003) and Bierbrauer et al. (2004) propose a three-state regime-switching model to describe the stochastic component of the spot price in the Nordic power exchange. To capture the cyclical behavior of the semiconductor business, Liu and Chyi (2006) use a Markov regime-switching model with two regimes representing expansion and contraction of semiconductor sales. For the Australian wholesale electricity market, Higgs and Worthington (2008) propose a three-state regime-switching model for electricity spot prices.

The lattice approach, which was first proposed by Cox et al. (1979), is one of the methods used to model the multi-period behavior of the regime-switching underlying variables. For example, Bollen (1998) proposes a pentanominal lattice approach to describe a two-state regime-switching model for a single underlying variable and then values European and American options. Wahab et al. (2010) develop a lattice approach for the valuation of swing options by modeling the average daily price of electricity using a three-state regime-switching model. Wahab and Lee (2009) generalize the lattice approach for multiple regime-switching variables with multiple regimes and then value European and American rainbow options.

\subsection{Dynamic programming approach}

A dynamic programming approach is a recursive approach to solving optimal decision problems, when the current decision influences the future payoff. Following the canonical form of Bellman's approach that given the current choice, the optimal strategy in the next period is chosen as if the entire analysis were to begin in the next period. The solution moves in a backward recursive direction, discounting the future values and folding them into the current decision. A dynamic programming approach is able to handle real options features efficiently. Intermediate values and 
decisions are transparent (Amram and Kulatilaka 1999).

\subsection{Summary}

This chapter outlines the overall scheme of the thesis. Modeling of stochastic processes along with their lattice representations are also discussed. The next chapter presents the valuation model for a natural gas-fired power plant with multiple turbines using spark spread and clean spark spread options. 


\section{Chapter 4}

\section{The Valuation of a Power Plant with Multiple Turbines using Spark Spread and Clean Spark Spread Options}

\subsection{Introduction}

This chapter presents methods to assess the value of a natural gas-fired power plant with multiple turbine in presence and absence of carbon allowance price. Turbines have different heat rates, generation capacities and carbon emission rates.

\subsection{Power plant characteristics}

The power plant is assumed to have multiple power generating gas turbine units. Let $G$ be the total number of gas turbines and $g=\{1,2, \ldots, G\}$. Each gas turbine has the minimum and maximum operating capacities of $\underline{q}_{g}$ and $\bar{q}_{g}$, respectively. These minimum and maximum capacities of a gas turbine are restricted mainly by the pressure ratio between the inlet and outlet of the turbine and the use of regenator (Boyce 2006).

Let $H\left(q_{g}\right)$ be the fuel input in MMBTu/hr for generating $q_{g} \mathrm{MW}$ of electricity from each turbine and $H\left(q_{g}\right)$ can be expressed as an input-output characteristic relationship (Wood and Wollenberg 1996)

$$
H\left(q_{g}\right)=a_{g}+b_{g} q_{g}+c_{g} q_{g}{ }^{2}
$$

Each coefficient of $H\left(q_{g}\right)$ is nonnegative, in which $a_{g}>0$ refers to the noload cost, $b_{g}>0$ denotes the fixed term of the incremental heat rate and $c_{g}>0$ represents that the cost function is convex (Tseng and Lin 2007; Tseng et al. 2009). For a power plant having $G$ turbines, the plant can be at 
any of $2^{G}$ states. Let $\mathbf{s}$ denote the possible states of the plant defined by the different combinations of 'on/off' status of turbines. For example, a power plant having two turbines can have four states denoted by $\mathbf{s}$, such that $\mathbf{s}=\left(\begin{array}{llll}s_{1} & s_{2} & s_{3} & s_{4}\end{array}\right)^{\prime}$, where $s_{1}=\left[\begin{array}{ll}0 & 0\end{array}\right]$ denotes that both Turbine 1 and Turbine 2 are at the 'off' state; $s_{2}=\left[\begin{array}{ll}1 & 0\end{array}\right]$ represents that Turbine 1 is 'on' but Turbine 2 is 'off'; and in similar fashion, $s_{3}=\left[\begin{array}{ll}0 & 1\end{array}\right]$, and $s_{4}=\left[\begin{array}{ll}1 & 1\end{array}\right]$. The prime notation refers to transposition of a matrix. Therefore, $\mathbf{s}$ is a $4 \times 2$ matrix as $\mathbf{s}=\left(\begin{array}{lllll}0 & 0 ; 1 & 0 ; 0 & 1 ; 1 & 1\end{array}\right)^{\prime}$. The flexibility of switching, in this study, is defined as the ability of switching from one state to another state of the power plant.

\subsection{Spark spread option}

The power plant owner buys the natural gas at a price of $P_{n}$ per MMBTu, burns and converts it into electric power and sells the electricity at a price of $P_{e}$ per MWh. The plant earns a spark spread profit (per hr) by running Turbine $g$ as:

$$
\begin{aligned}
\text { Spark spread } & =q_{g} P_{e}-H\left(q_{g}\right) P_{n}-q_{g} v_{g} \\
& =q_{g} P_{e}-\left(a_{g}+b_{g} q_{g}+c_{g} q_{g}{ }^{2}\right) P_{n}-q_{g} v_{g}
\end{aligned}
$$

where $v_{g}$ is the operational and maintenance cost per MWh and $H\left(q_{g}\right)$ is the fuel input in MMBTu/hr for generating $q_{g} \mathrm{MW}$ of electricity. The optimal quantity of generation, $q_{g}^{*}$, can be obtained by differentiating Equation (4.2) with respect to $q_{g}$ and setting it to zero. The optimal generation, $q_{g}^{*}$, is obtained as:

$$
q_{g}^{*}=\frac{1}{2 c_{g}}\left(\frac{P_{e}-v_{g}}{P_{n}}-b_{g}\right)
$$

Since a gas turbine has the minimum and maximum operating capacities of $\underline{q}_{g}$ and $\bar{q}_{g}$ respectively, the optimal feasible power generation, $\hat{q_{g}}$, becomes:

$$
\hat{q_{g}}=\max \left(\underline{q}_{g}, \min \left(q_{g}^{*}, \bar{q}_{g}\right)\right) .
$$

The generation quantity, $q_{g}$, is then replaced by $\hat{q_{g}}$ in Equation (4.2). Therefore, the spark spread, $\Omega_{g}$, from each turbine of a power plant operating $h$ hours per day can be expressed as:

$$
\Omega_{g}=h\left\{\hat{q_{g}} P_{e}-\left(a_{g}+b_{g} \hat{q_{g}}+c_{g}{\hat{q_{g}}}^{2}\right) P_{n}-\hat{q_{g}} v_{g}\right\}
$$


Now the value of spark spread at different states of $\mathbf{s}$, can be expressed as:

$$
\boldsymbol{\Pi}_{\mathbf{s}}=\mathbf{s} \cdot\left(\boldsymbol{\Omega}_{g}\right)^{\prime}
$$

As an example, for a power plant with two turbines, the values of the spark spread at different states can be expressed as:

$$
\boldsymbol{\Pi}_{s}=\left(\begin{array}{llll}
0 & 1 & 0 & 1 \\
0 & 0 & 1 & 1
\end{array}\right)^{\prime} \cdot\left(\begin{array}{l}
\Omega_{1} \\
\Omega_{2}
\end{array}\right)
$$

The spark spread options, $\pi_{\mathbf{s}}$, therefore, can be expressed as:

$$
\pi_{\mathbf{s}}=\max \left(0, \boldsymbol{\Pi}_{\mathbf{s}}\right)
$$

\subsection{Clean spark spread option}

The clean spark spread (per hr) can be expressed by deducting the carbon allowance price from the spark spread and can be written as:

$$
\text { Clean spark spread }=q_{g_{c}} P_{e}-\left(a_{g}+b_{g} q_{g_{c}}+c_{g} q_{g_{c}}^{2}\right) P_{n}-q_{g_{c}} v_{g}-\mathcal{R}_{g} q_{g_{c}} P_{c}
$$

where, $P_{c}$ is the carbon allowance price per $\mathrm{tCO}_{2}, \mathcal{R}_{g}$ is the emission rate in $\mathrm{tCO}_{2} / \mathrm{MWh}$, and the subscript, $c$, denotes the 'clean' spark spread, that is, the spark spread that considers the 'carbon' cost. The optimal generation, $q_{g_{c}}^{*}$, now becomes:

$$
q_{g_{c}}^{*}=\frac{1}{2 c_{g}}\left(\frac{P_{e}-v_{g}-\mathcal{R}_{g} P_{c}}{P_{n}}-b_{g}\right) .
$$

This optimal generation quantity, $q_{g_{c}}^{*}$, must be within the maximum and minimum generation capacities of each turbine. Therefore, the feasible power generation, $\hat{q}_{g_{c}}$, from each turbine is as follows:

$$
\hat{q_{g_{c}}}=\max \left(\underline{q}_{g}, \min \left(q_{g_{c}}^{*}, \bar{q}_{g}\right)\right)
$$

The generation quantity, $q_{g_{c}}$, is then substituted by $\hat{q}_{g_{c}}$ in Equation (4.9) to obtain the clean spark spread value. Therefore, for a power plant operating $h$ hours per day, the clean spark spread, $\Omega_{g_{c}}$, is given by:

$$
\Omega_{g_{c}}=h\left\{\hat{q_{g_{c}}} P_{e}-\left(a_{g}+b_{g}{\hat{q_{c}}}_{g_{c}}+c_{g} \hat{q}_{g_{c}}^{2}\right) P_{n}-\hat{q}_{g_{c}} v_{g}-\mathcal{R}_{g} \hat{q}_{g_{c}} P_{c}\right\}
$$


where, the subscript, $c$, denotes the 'clean' spark spread. Following the expression for spark spread options in Section 4.3, the clean spark spread option at different states can be obtained as:

$$
\left(\pi_{c}\right)_{\mathbf{s}}=\max \left(0,\left(\boldsymbol{\Pi}_{c}\right)_{\mathbf{s}}\right)
$$

\subsubsection{An exhibit}

This section provides some insights by illustrating an example of a natural gas-fired power plant having two turbines with different emission rates, input-output characteristics, and generation capacities. The parameters are shown in Table 4.1. ${ }^{\mathrm{a}}$ While electricity price is linearly varied from 33 to 39 Euro/MWh, natural gas and carbon allowance prices are kept constant at 3.63 Euro/MMBtu and 6 Euro/ $\mathrm{tCO}_{2}$, respectively.

Table 4.1: Turbine characteristics of a power plant

\begin{tabular}{lll}
\hline Parameters & Gas turbine $1(g=1)$ & Gas turbine $2(g=2)$ \\
\hline Input-output & $a_{1}=70$ & $a_{2}=75$ \\
coefficient of & $b_{1}=8.0$ & $b_{2}=7.5$ \\
$H\left(q_{g}\right)$ & $c_{1}=0.00115$ & $c_{2}=0.00130$ \\
\hline Maximum capacity, $\bar{q}_{g}$ & $300 \mathrm{MW}$ & $350 \mathrm{MW}$ \\
Minimum capacity, $\underline{q}_{g}$ & $5 \mathrm{MW}$ & $5 \mathrm{MW}$ \\
Operating \& maintenance cost, $v_{g}$ & $5 / \mathrm{MWh}$ & $4 / \mathrm{MWh}$ \\
Carbon emission rate, $\mathcal{R}_{g}$ & $0.45 \mathrm{tCO}_{2} / \mathrm{MWh}$ & $0.30 \mathrm{tCO}_{2} / \mathrm{MWh}$ \\
\hline
\end{tabular}

Variations in input-output characteristics of turbines: Figure 4.1 shows the input-output characteristic curves of both Turbine 1 and Turbine 2. The corresponding average heat rates for both turbines are also depicted. It is observed that Turbine 2 has a lower average heat rate than that of Turbine 1. Since the efficiency of a turbine is the inverse of the heat rate, Turbine 2 is more efficient than Turbine 1.

Variations in power generation with and without carbon allowances: Figure 4.2 shows how the presence and absence of carbon allowance cost affects power generation from different turbines of a power plant. The curves represent the feasible power generation, $\hat{q_{g}}$, obtained from Equations (4.3)-(4.4) in Section 4.3 in absence of carbon allowance prices. It is observed that power generation from Turbine 2 is more than that of Turbine 1 . The reason is that Turbine 2 has a lower heat inputoutput characteristic line and hence possesses a higher efficiency than that of Turbine 1. However, under $\mathrm{CO}_{2}$ emission restrictions, the quantity, $\hat{q}_{g_{c}}$, is reduced for both Turbine 1 and Turbine 2 .

\footnotetext{
${ }^{a}$ In Wood and Wollenberg (1996), for a coal-fired power generation unit with the maximum and minimum output of $600 \mathrm{MW}$ and $200 \mathrm{MW}$, respectively, the coefficients are assumed as: $a=510, b=7.2$, and $c=0.00142$; and for an oil-fired power generating unit with the maximum and minimum output of $200 \mathrm{MW}$ and 50 MW, respectively, the coefficients are assumed as: $a=78, b=7.97$, and $c=0.00482$. Tseng and Lin (2007) also use a quadratic input-output function with $a=540, b=9.223$, and $c=0.00234$ for a gas-fueled power plant with the maximum and minimum capacity of $700 \mathrm{MW}$ and $225 \mathrm{MW}$, respectively.
} 


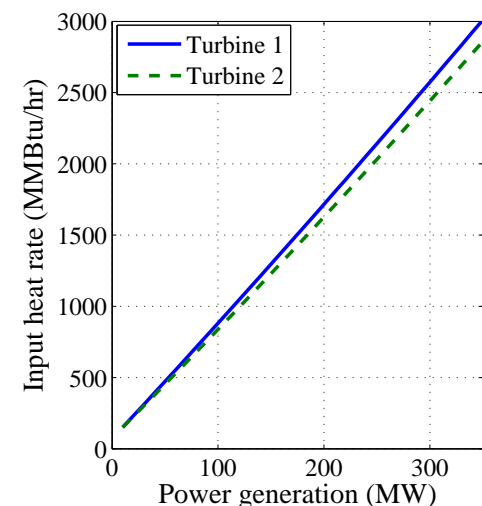

(a)

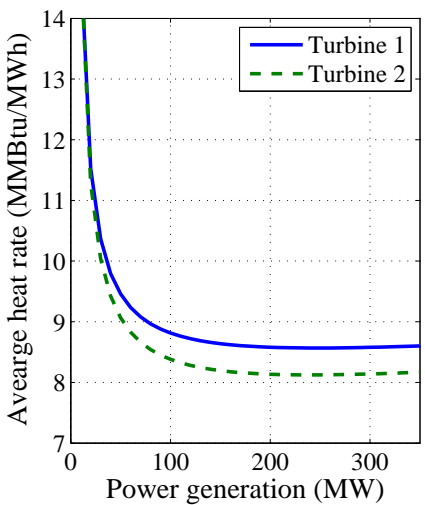

(b)

Figure 4.1: (a) Input-output characteristic curves and (b) corresponding average heat rates of turbines.

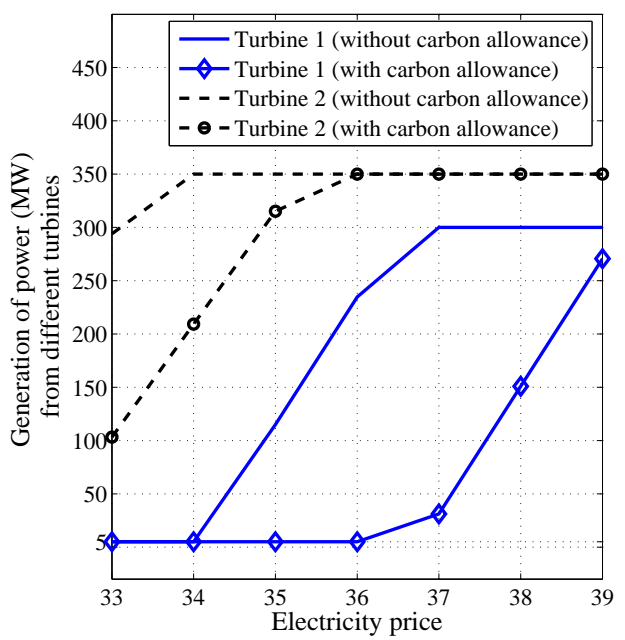

Figure 4.2: Variations in generation of power (MW) from two turbines in absence and presence of carbon allowance price.

Variations in spark and clean spark spread values: Figure 4.3 shows the values of spark and clean spark spread profits along with their respective real options values under different states of the power plant. It is observed that in case of spark spread (see the left-side of Figure 4.3), Turbine 2 offers higher profits for a range of electricity prices between 34 to 36 Euro/MWh. When the electricity price crosses 36 Euro/MWh, operating both Turbine 1 and Turbine 2 provides a higher profit. However, in presence of carbon price, operating only Turbine 2 offers a higher profit until the electricity price hits 39 Euro/MWh (see the right-side of Figure 4.3). It reflects that under the environmental restriction, at a lower electricity price, operating both turbines may induce more emission cost.

This example demonstrates how the flexibility of having two turbines with different emission rates, input-output characteristics and power generation capacities affects the cashflows in presence and absence of $\mathrm{CO}_{2}$ emission allowance price. These results, however, did not consider the stochastic 


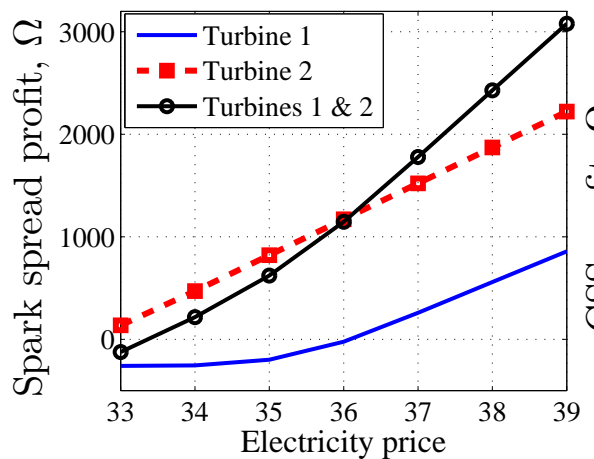

(a)

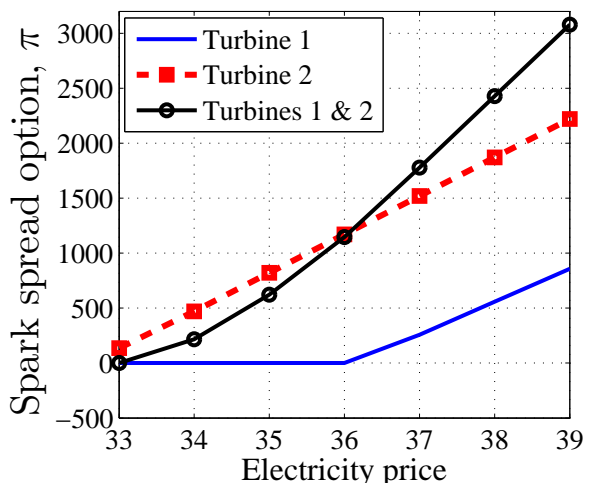

(c)

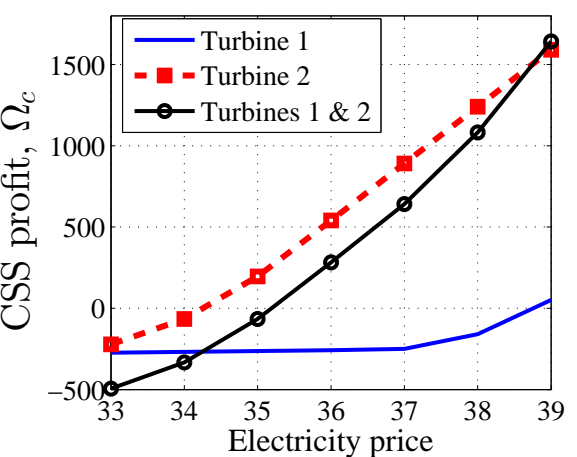

(b)

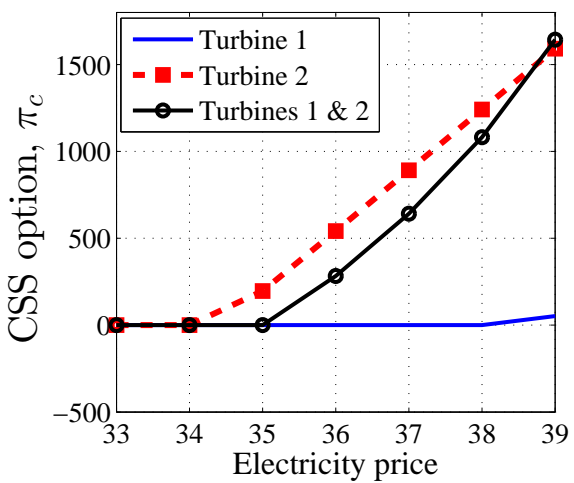

(d)

Figure 4.3: (a) Spark spread, $\Omega$, and (b) clean spark spread, $\Omega_{c}$, profits; (c) Spark spread, $\pi$, and (d) clean spark spread, $\pi_{c}$, options under different operating modes of turbines.

behavior of prices. In this thesis, the valuation of the power plant is considered under the stochastic behaviors of electricity, natural gas and carbon prices. Details of the approach are discussed in the following sections.

\subsection{The valuation of the power plant}

The value of the power plant based on spark spread option, $\mathbf{W}_{t}$, can be determined as a summation of the current total options plus the expected total options from the next period by using a backward dynamic programming approach as follows:

$$
\mathbf{W}_{t}=\pi_{t}+e^{-r(\Delta t)} \mathbf{E}_{t}\left[\mathbf{W}_{t+1}\right],
$$

where $r$ is the risk-free rate. When the behaviors of electricity, natural gas and carbon prices are modeled based on futures prices, the expected value of the plant can be calculated using the risk-free discount rate, $r$ (Abadie, 2015). Similarly, the value of the plant based on clean spark 
spread options, $\left(\mathbf{W}_{c}\right)_{t}$, can be expressed as:

$$
\left(\mathbf{W}_{c}\right)_{t}=\left(\pi_{c}\right)_{t}+e^{-r(\Delta t)} \mathbf{E}_{t}\left[\left(\mathbf{W}_{c}\right)_{t+1}\right]
$$

The value of the power plant based on spark spread options along with operational flexibility, $\mathbf{F}_{t}$, is:

$$
\mathbf{F}_{t}=\pi_{t}+e^{-r(\Delta t)} \max \left\{\mathbf{E}_{t}\left[\mathbf{F}_{t+1}+C\left((s)_{t, t+1}\right)\right]\right\},
$$

where $C\left((s)_{t, t+1}\right)$ is the switching cost from one state to another state of the power plant. For example, if startup and shutdown costs for Turbine 1 are $C_{u 1}$ and $C_{d 1}$, respectively; and for Turbine 2 are $C_{u 2}$ and $C_{d 2}$, respectively, then $C\left((s)_{t, t+1}\right)$ is a $4 \times 4$ matrix as:

$$
C\left((s)_{t, t+1}\right)=\left(\begin{array}{cccc}
0 & C_{u 1} & C_{u 2} & C_{u 1}+C_{u 2} \\
C_{d 1} & 0 & C_{d 1}+C_{u 2} & C_{u 2} \\
C_{d 2} & C_{u 1}+C_{d 2} & 0 & C_{u 1} \\
C_{d 1}+C_{d 2} & C_{d 2} & C_{d 1} & 0
\end{array}\right) .
$$

Operating constraints such as, startup, shutdown, ramp time and costs, minimum up and down time, are usually measured hourly (Johnson and Miranda, 2003). However, in the above cost matrix, the switching costs are assumed to represent average daily costs covering all costs related to daily operating constraints for a turbine. Similarly, the value of the power plant based on clean spark spread options with operational flexibility, $\left(\mathbf{F}_{c}\right)_{t}$, can be expressed as:

$$
\left(\mathbf{F}_{c}\right)_{t}=\left(\pi_{c}\right)_{t}+e^{-r(\Delta t)} \max \left\{\mathbf{E}_{t}\left[\left(\mathbf{F}_{c}\right)_{t+1}+C\left((s)_{t, t+1}\right)\right]\right\}
$$

\subsection{Price processes}

For the valuation of a power plant based on spark spread options, it is necessary to generate prices of electricity and natural gas. Commodity prices, such as oil, usually follow a mean-reverting process. As discussed in Section 3.2, the price may fluctuate randomly up and down in the short run, but in the long run it moves towards the mean price value.

Prices of electricity and natural gas are assumed to follow mean-reverting processes. Abadie and Chamorro (2008a) provide an argumentive discussion on it. For the clean spark spread option, in addition to electricity and natural gas prices, the carbon allowance price also needs to be simulated. While Huang (2010) models the carbon allowance price as a mean-reverting process, Seifert et al. (2008) suggest that the $\mathrm{CO}_{2}$ emission allowance price follows a geometric Brownian motion. Keeping both arguments in consideration, two scenarios are considered for the clean spark spread 
option based valuation. In one scenario, all three prices of electricity, natural gas and $\mathrm{CO}_{2}$ allowance prices are assumed to follow mean-reverting processes. In another scenario, prices of electricity and natural gas are modeled as mean-reverting processes, while the $\mathrm{CO}_{2}$ allowance price is modeled as a geometric Brownian motion. In this study, daily prices of electricity, natural gas and $\mathrm{CO}_{2}$ are obtained from European Energy Exchange (EEX) and Figure 4.4 shows futures prices for electricity, natural gas and carbon prices from January 1, 2013 to June 3, 2013.
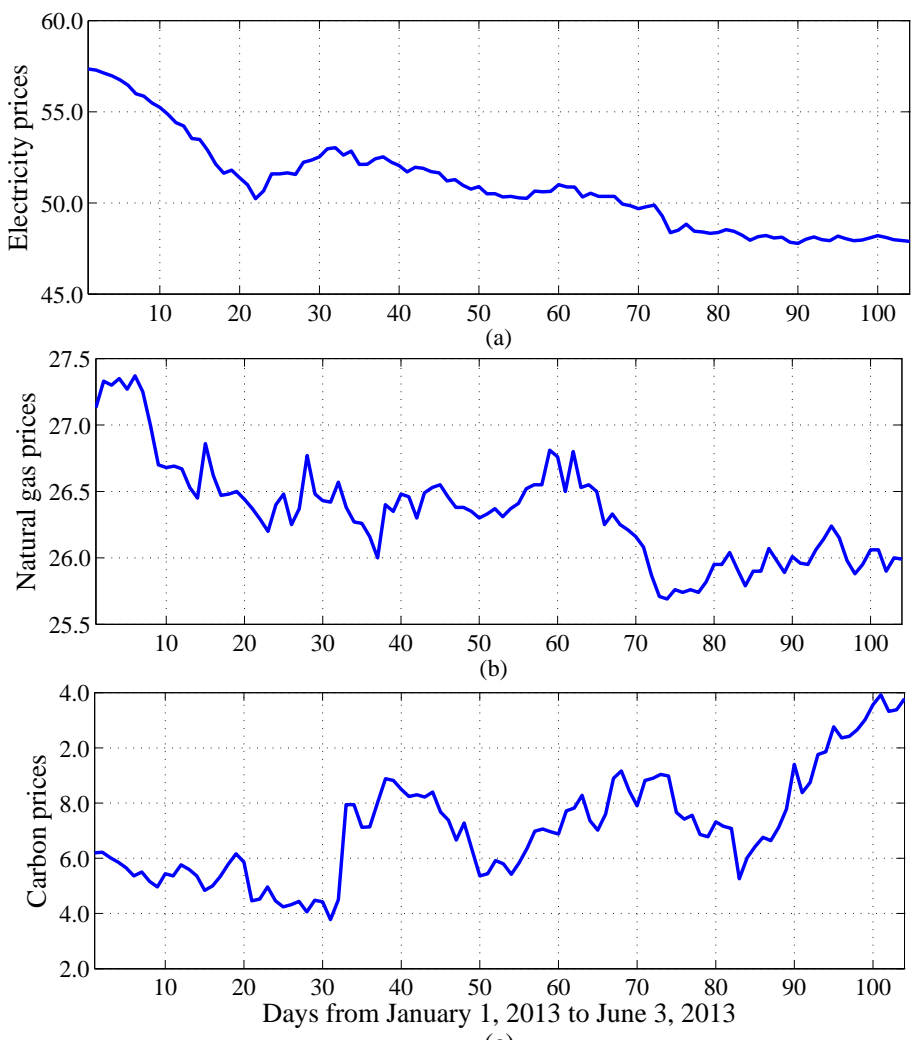

(c)

Figure 4.4: Futures prices (daily): (a) electricity, (b) natural gas and (c) carbon allowance.

\subsubsection{An estimation of mean-reverting process parameters}

A mean-reverting process can be discretized into the first order auto-regression, $\mathrm{AR}(1)$, process (see Appendix D). For example, in the case of the natural gas price process, Equation (3.1) can be discretized and expressed as: $\frac{\Delta x_{t}}{\Delta t}=\alpha+\varrho x_{t}+\epsilon_{t}$, where $\alpha$ is the intercept, $\Delta t$ is the discretized time interval, $\varrho$ is the slope of the linear regression line drawn by minimizing the sum of the squared residuals, and $\epsilon_{t} \sim \mathrm{N}\left(0, \sigma_{x}^{2}\right)$ are the residuals (Brooks, 2008; Elias et al., 2014). Table 4.2 shows these coefficients of the discretized mean-reverting processes as respective $\operatorname{AR}(1)$ processes, obtained by using the MATLAB statistical function 'regress'. 
Table 4.2: Mean-reverting parameters for price processes

\begin{tabular}{lcrrrr}
\hline Prices & Coeff. & Value & Std. err. & t-stats & p-value \\
\hline Natural gas & $\alpha$ & 41.8289 & 18.0042 & 2.3233 & 0.0222 \\
& $\varrho$ & -20.5169 & 8.8087 & -2.3292 & 0.0218 \\
\hline Electricity & $\alpha$ & 28.2897 & 11.3648 & 2.4892 & 0.0144 \\
& $\varrho$ & -7.3091 & 2.8913 & -2.5279 & 0.0130 \\
\hline Carbon allowance & $\alpha$ & 30.5811 & 13.2096 & 2.3151 & 0.0223 \\
& $\varrho$ & -21.2626 & 8.7340 & -2.4345 & 0.0167 \\
\hline
\end{tabular}

From Table 4.2, parameters obtained for the natural gas price process are: $\kappa_{x}=|\varrho|=20.5169$, $\bar{x}=\frac{\alpha}{|\varrho|}=2.0388, \sigma_{x}=\operatorname{std}\left(\epsilon_{t}\right) \times \sqrt{\Delta t}=0.08156$, where 'std' stands for the standard deviation. Similarly, for the electricity price process, $\bar{y}=3.8705, \kappa_{y}=7.3091, \sigma_{y}=0.08947$; and for the carbon allowance price process, $\bar{z}=1.4383, \kappa_{z}=21.2626, \sigma_{z}=1.1708$. Correlation coefficients obtained from the residuals of respective price processes are: $\rho_{x y}=0.3128, \rho_{y z}=0.5789$, and $\rho_{z x}=0.1994$. The positive correlations indicate that natural gas, electricity and carbon allowance prices move in the same direction. Linn et al. (2014) also conclude that lower natural gas prices induce lower electricity prices and lower emissions. With $\Delta t$ equals to $\frac{1}{250}$, the units of the parameters are measured in years, for example, the yearly volatility of electricity prices, $\sigma_{y}$, is approximately $9 \%$, while the yearly volatility of natural gas futures prices, $\sigma_{x}$, is approximately $8 \%$. An important property of the mean-reversion process is the half life, $t_{1 / 2}=\frac{\ln 2}{\kappa}$. It is the time taken by the price to attain the half way of the long-term mean price from its current price, as if no random shock were present (Rogers 2002). The higher the mean-reversion speed is, the smaller the half life is. Therefore, the half lives, $(\ln 2) / \kappa$, of the electricity, natural gas and carbon prices are, 0.095, 0.034, and 0.033 years (or 23.8, 8.5, and 8.3 days), respectively.

The drift, $\mu_{z}$, and the variance, $\sigma_{z}^{2}$, when $\mathrm{CO}_{2}$ price is modeled as a Brownian motion, are presented in Table 4.3. The correlation coefficients between residuals of electricity and carbon prices and between natural gas and carbon allowance prices are obtained as, 0.5590, and 0.1795, respectively.

Table 4.3: Geometric Brownian motion parameters for $\mathrm{CO}_{2}$ allowance price

\begin{tabular}{lllll}
\hline Parameter & Coeff. & Std. err. & t-stats & p-value \\
\hline Drift & -1.27 & 0.1189 & -10.6783 & 0.00 \\
Variance & 1.4569 & 0.2040 & 7.1414 & 0.00 \\
\hline
\end{tabular}




\subsection{An illustrative example}

This section illustrates how the expected spark spread option based values at different states of a power plant are calculated depending on the movements of natural gas and electricity prices along a bivariate lattice. The example is executed for a short period of three days. The initial prices of natural gas and electricity are assumed to be $6 €$ and $60 €$, respectively. Figure 4.5 shows that the price movements of natural gas and electricity. For example, at $t=2$ in Figure 4.5, the up movements of both natural gas and electricity prices are represented by node 'c'. Similarly, node 'b' represents the down and up movements of natural gas and electricity prices, respectively. Spark spread option values corresponding to the natural gas and electricity prices at different states of the power plant are shown in Figure 4.6. The expected values based on spark spread option are presented in Figure 4.7. The branch probabilities are obtained by using Equations (3.18-3.21). These probabilities, for an example, from node ' $\mathrm{b}$ ' at $t=2$ to the next period at $t=3$ are: $p_{d u}^{*}=0$ to node 'f', $p_{u u}^{*}=0.4126$ to node 'g', $p_{u d}^{*}=0.5874$ to node 'i', and $p_{d d}^{*}=0$ to node ' $\mathrm{h}$ '. The expected values at different states are calculated by using Equation (4.14). For example, in Figure 4.7 at $t=2$ at node ' $\mathrm{b}$ ' in State 2, the expected value of $58959.40 €$ is obtained by using Equation (4.14).

$$
\begin{aligned}
58959.40=30731.00 & +\exp \{-0.05 \times(1 / 250)\} \times\{(0 \times 35401.84)+(0.4126 \times 31322.73) \\
& +(0 \times 30143.61)+(0.5874 \times 26064.50)\}
\end{aligned}
$$



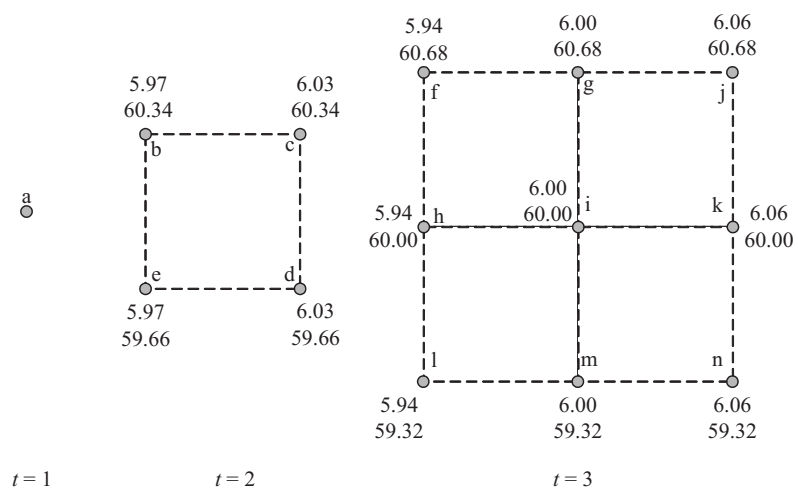

Figure 4.5: An example of movements of natural gas prices and electricity prices along a bivariate lattice from layer $t=1$ to $t=3$.

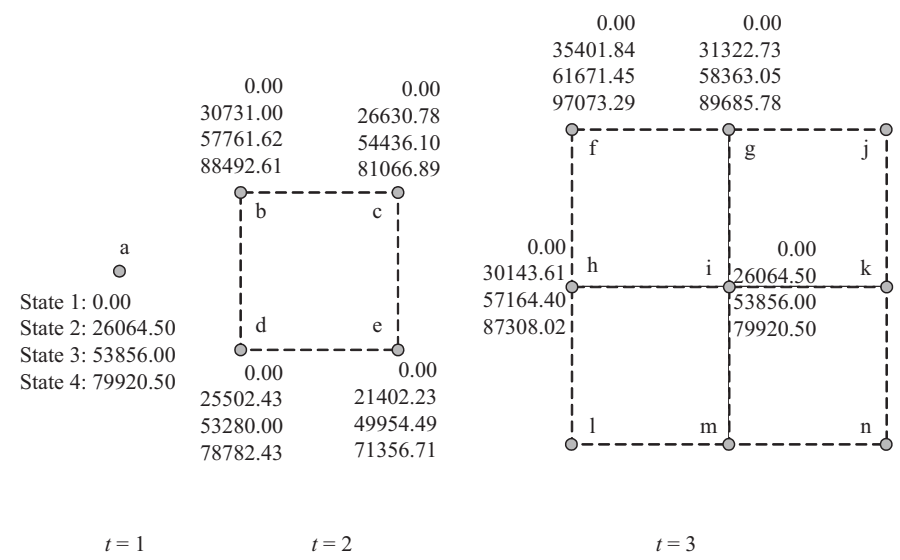

Figure 4.6: Values of the spark spread options at different states of the power plant corresponding to the prices in Figure 4.5.

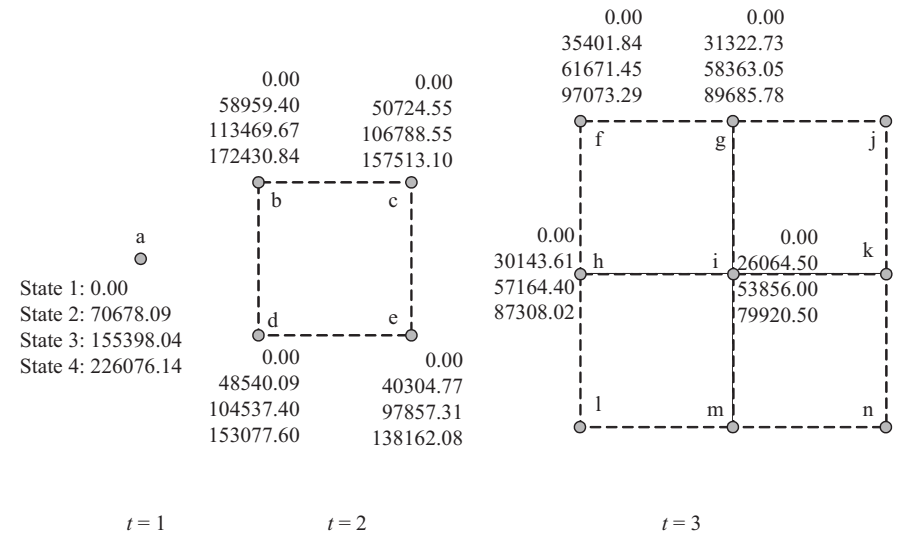

Figure 4.7: Expected spark spread option values of the power plant at different states of the power plant corresponding to the values in Figure 4.6. 


\subsection{Numerical results}

A power plant with heavy duty frame-type gas turbines is chosen for a numerical analysis of the proposed model. The mechanical characteristics of gas turbines used in this thesis are adopted from the Gas Turbine Engineering Handbook by Boyce (2006). The capacities of such turbines range from $3 \mathrm{MW}$ to $480 \mathrm{MW}$. Operating hours of the plant are assumed to be $h=22$ hours per day. A typical modern gas turbine usually takes 6-10 minutes to start-up (ramp-up) and shut-down (ramp-down) (Boyce 2006). The average operating hour, $h$, per day is assumed to take into account of all necessary ramp-up, ramp down, and plant maintenance times per day, on an average. The input-output characteristic coefficients of $H\left(q_{g}\right)$, the maximum and minimum capacities of turbines are shown in Table 4.1 in Section 4.4.1. Table 4.4 shows the startup and shutdown costs (in Euro) of the power plant. The valuation period is assumed to be 90 days. The annual discount rate is assumed to be $5 \%$. Sensitivity tests are performed by varying between $-10 \%$ and $+10 \%$ of the base values of volatilities and mean-reverting speeds stated in Section 4.6.1.

Table 4.4: Turbine startup and shutdown costs in Euro

\begin{tabular}{lrr}
\hline & Turbine 1 & Turbine 2 \\
\hline Startup & 100 & 150 \\
Shutdown & 400 & 500 \\
\hline
\end{tabular}

\subsubsection{Expected spark and clean spark spread option values}

The expected spark spread and clean spark spread values with and without flexibility of switching among the states are shown in Figure 4.8. Figure 4.8(a) shows the expected values of the power plant at different states without flexibility. As stated in Section 4.2, State 1 refers to the status of the power plant when both turbines are in 'off' state, State 2 indicates that Turbine 1 is in 'on' state and Turbine 2 is in 'off' state, State 3 refers to Turbine 1 is in 'off' state and Turbine 2 is in 'on' state, and State 4 represents that both turbines are in 'on' states. The notion of the expected values without flexibility is that these states, State 1, State 2, State 3, and State 4, prevail throughout the valuation planning period and there occurs no switching among these states. The objective is to measure a reference expected value of the power plant based on spark spread option at a different state. This is the reason that the expected value without flexibility at State 1 is zero. Note that the expected value of the power plant is the maximum of expected values over all states. Therefore, the expected spark spread option value of the power plant without flexibility is $801442.47=\max (0,160773.12,720358.67,801442.47)$.

Figure 4.8(b) shows the expected values of the power plant with switching flexibility. The 
flexibility here refers to the ability to switch from one state to another state. For example, if the plant is in State 1 at $t=1$, the plant can remain in State 1, or switch to State 2, or State 3, or State 4 at $t=2$ depending on the movement of prices and so on. The expected values with flexibility are calculated by using Equation (4.16). The optimal path of power plant operation with two turbines can be traced from the lattice, in which flexibility of switching is embedded. The flexibility of switching adds value to the power plant. This value addition due to flexibility is the highest at State 1 and the lowest at State 4. It is observed from Figure 4.8(b) that at State 1, the expected value of the plant with flexibility is $799625.09 €$. The expected spark spread option value of the power plant with flexibility is $879795.55=\max (799625.09,825789.57,853631.07,879795.55)$.

It is also observed that purchasing $\mathrm{CO}_{2}$ allowance reduces the value of the power plant. From Figure 4.8(a), it is observed that the expected CSS value at State 4 is lower than that of at State 3. Because at State $4, \mathrm{CO}_{2}$ emissions from both turbines induce more carbon cost than that of at State 3. Table 4.5 shows the percentage increase/reduction in the values of the power plant at different states due to flexibility and $\mathrm{CO}_{2}$ cost.

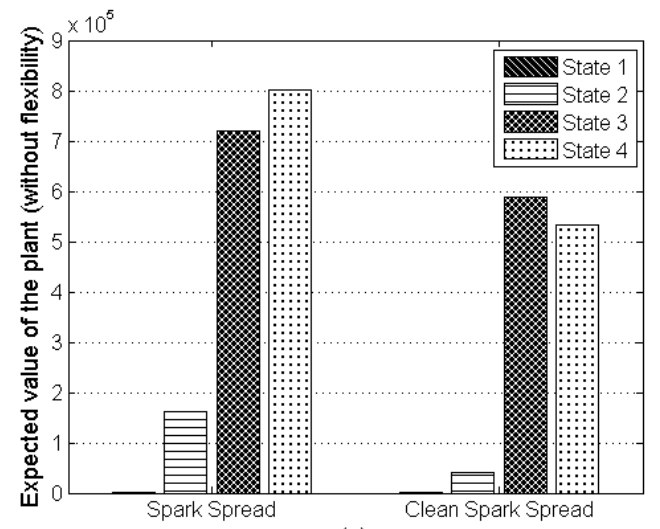

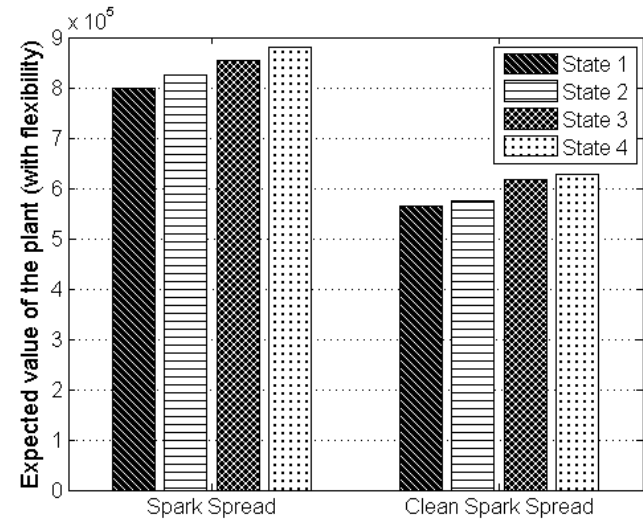

Figure 4.8: Expected spark and clean spark spread values of the plant at different operating states of the plant: (a) without flexibility and (b) with flexibility.

Table 4.5: Expected SS and CSS values with and without flexibilities at different states of the power plant

\begin{tabular}{c|ccc|ccc|cc}
\hline & \multicolumn{3}{|c|}{} & \multicolumn{3}{c|}{$\begin{array}{c}\text { Expected CSS } \\
\left(\mathrm{CO}_{2} \text { : }\right.\end{array}$} & \multicolumn{3}{c}{ Vean-reverting $)$} & \multicolumn{2}{c}{$\begin{array}{c}\text { Value reduction } \\
\text { due to } \mathrm{CO}_{2}\end{array}$} \\
\hline States & No flexibility & Flexibility & \% increase & No flexibility & Flexibility & $\%$ increase & No flexibility & flexibility \\
\hline State 1 & 0 & 799625.09 & - & 0 & 560920.26 & - & - & $29.85 \%$ \\
State 2 & 160773.12 & 825789.57 & $413.63 \%$ & 41552.93 & 572438.24 & $1277.61 \%$ & $74.15 \%$ & $30.68 \%$ \\
State 3 & 720358.67 & 853631.07 & $18.50 \%$ & 584630.43 & 613309.23 & $4.91 \%$ & $18.84 \%$ & $28.15 \%$ \\
State 4 & 801442.47 & 879795.55 & $9.78 \%$ & 530394.65 & 624827.21 & $17.80 \%$ & $33.82 \%$ & $28.98 \%$ \\
\hline
\end{tabular}

As stated in Section 4.4.1, Turbine 1 with a higher average heat rate is less efficient than Turbine 2. For this reason, the percentage increase in the expected SS value at State 2 attains a higher value 


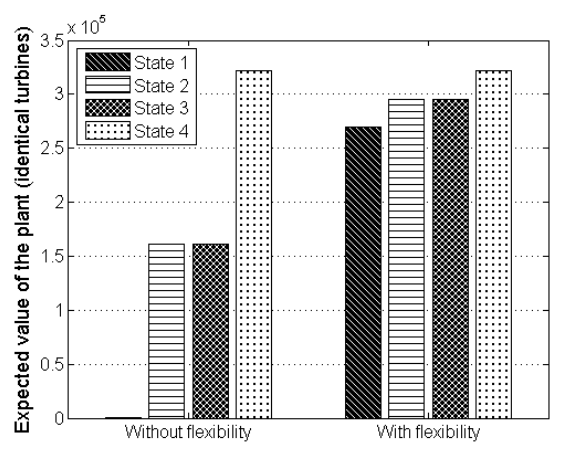

Figure 4.9: Expected spark spread option based values of the power plant having two identical gas turbines similar to Turbine 1.

of $413.63 \%\left(=\frac{825789.57-160773.12}{160773.12}\right)$, whereas the percentage increase in the expected SS value at State 3 is $18.50 \%\left(=\frac{853631.07-720358.67}{720358.67}\right)$. Also note that the expected CSS value at State 4, in absence of switching flexibility, is approximately, $5.30 \times 10^{5}$, which is lower than that of, $5.85 \times 10^{5}$, at State 3. Because operating both turbines (at State 4) induces more $\mathrm{CO}_{2}$ cost than operating only Turbine 2 (at State 3 ) and Turbine 2 has a lower emission rate of $0.30 \mathrm{tCO}_{2} / \mathrm{MWh}$ in comparison to Turbine 1 with an emission rate of $0.45 \mathrm{tCO}_{2} / \mathrm{MWh}$. The reduction in the expected values under $\mathrm{CO}_{2}$ emission restriction is also shown in Table 4.5. It is observed that the percentage reduction in the expected CSS value with flexibility at State 4 is $28.98 \%\left(=\frac{879795.55-624827.21}{879765.55}\right)$.

Figure 4.9 shows the expected spark spread option based values of the power plant, if the plant has two identical gas turbines with the same mechanical characteristics. Figure 4.9 represents the expected values, if Turbine 2 is replaced by Turbine 1 . That is, the power plant now possesses two turbines same as Turbine 1. The observations show that the expected value of the plant is reduced because of using Turbine 1, which has a higher heat rate.

A comparison between the effect of modeling $\mathrm{CO}_{2}$ price as a mean-reverting and a geometric Brownian motion is shown in Figure 4.10. The expected value of the power plant is higher when $\mathrm{CO}_{2}$ price is assumed to follow a geometric Brownian motion in comparison to the expected value when $\mathrm{CO}_{2}$ price is modeled as a mean-reverting process. Note that for the valuation of spark spread option, Deng et al. (2001) also show that modeling price processes under the geometric Brownian motion assumption overvalues the option in comparison to the modeling under the mean-reverting price process assumption. This is because the volatility of a geometric Brownian motion grows infinitely while the volatility of a mean-reverting process is bounded and in the long run decays to $\frac{\sigma^{2}}{2 \kappa}($ see Equation (A.18)). 

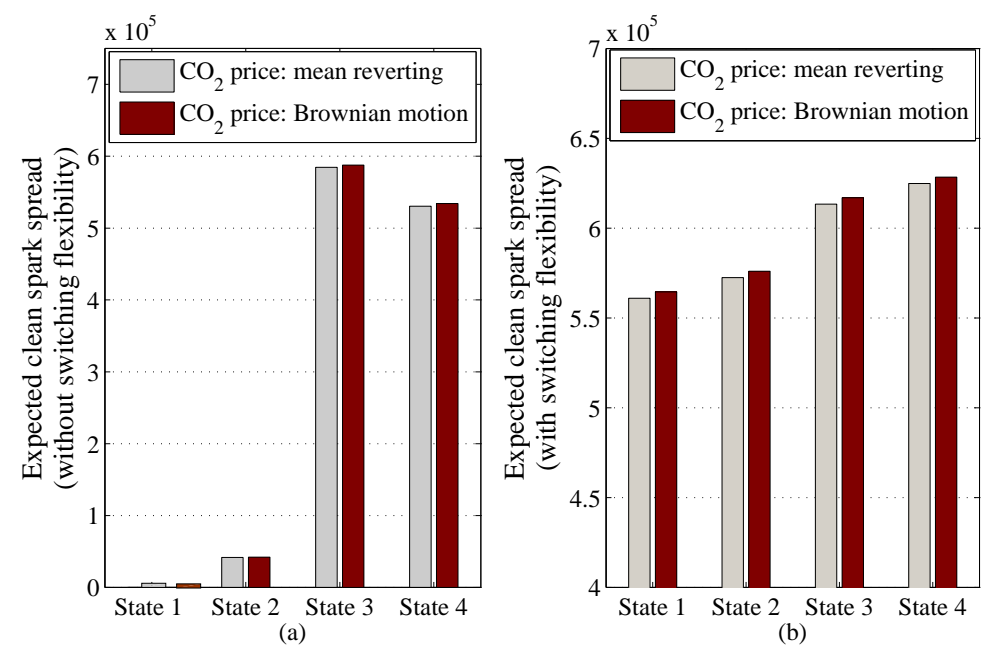

Figure 4.10: Expected clean spark spread values using two different carbon price modeling approaches: (a) without flexibility, and (b) with flexibility.

\subsubsection{Sensitivity to price volatilities}

A power plant can reap the benefit of higher volatilities in electricity price by exercising the options to operate at higher electricity prices. The sensitivity of the expected spark and clean spark spread values of the power plant to changes in electricity price volatilities is demonstrated in Figure 4.11. It is observed that the expected value of the power plant increases with the increase in electricity price volatilities. Figure 4.11 also illustrates that the operational flexibility of switching adds value to the power plant. Another point to note is that the expected values at State 3 change more rapidly than those of at State 2. This reflects that Turbine 2 is more sensitive to the volatility changes in electricity price than that of Turbine 1 . The reason is that Turbine 2 has a higher efficiency with a lower carbon emission rate and also with a lower maintenance cost. Figure 4.12 shows the effect of changes in the volatility of electricity price on the expected clean spark spread option value with respect to $\mathrm{CO}_{2}$ price process modeling approaches. It is observed that the expected clean spark spread value is higher when the $\mathrm{CO}_{2}$ emission allowance prices are modeled as a Brownian motion.

Figure 4.13 shows the effect of natural gas price volatilities on the expected SS and CSS values. A higher gas price volatility induces a higher uncertainty in the cost component of both SS and CSS options. The higher volatilities in cost component, therefore, tend to pull down the value of the plant as opposed to higher electricity price volatilities tend to increase the expected value of the plant. Consequently, Figure 4.13 shows that an increase in the volatility of natural gas price tends to reduce the power plant value. Due to the similar reason, Figure 4.14 shows that the expected clean spark spread values of the power plant decrease with an increase in $\mathrm{CO}_{2}$ price volatilities. 

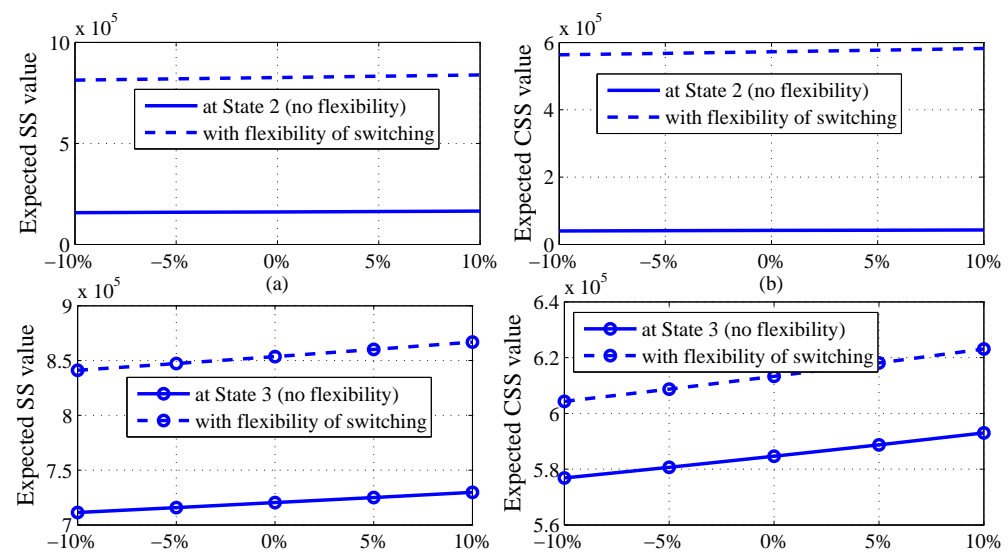

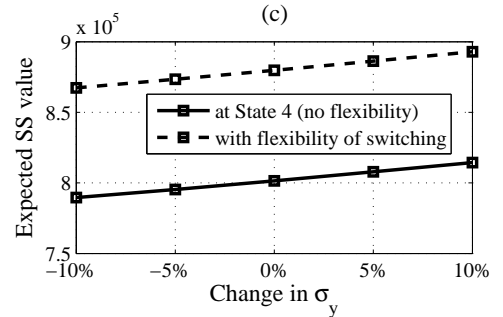

(e)

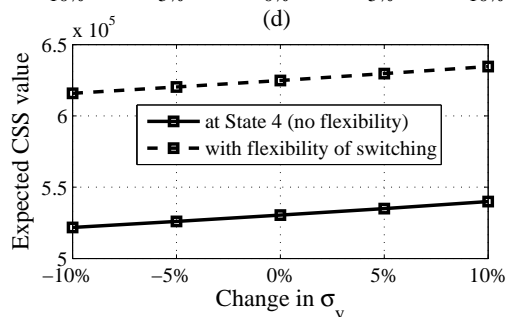

(f)

Figure 4.11: Expected spark and clean spark spread values of the power plant at different volatilities of electricity price: (a) State 2 (without carbon cost), (b) State 2 (with carbon cost), (c) State 3 (without carbon cost), (d) State 3 (with carbon cost), (e) State 4 (without carbon cost), and (f) State 4 (with carbon cost).

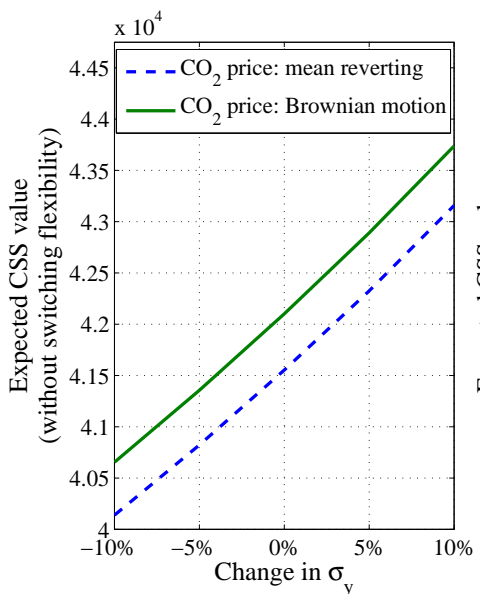

(a)

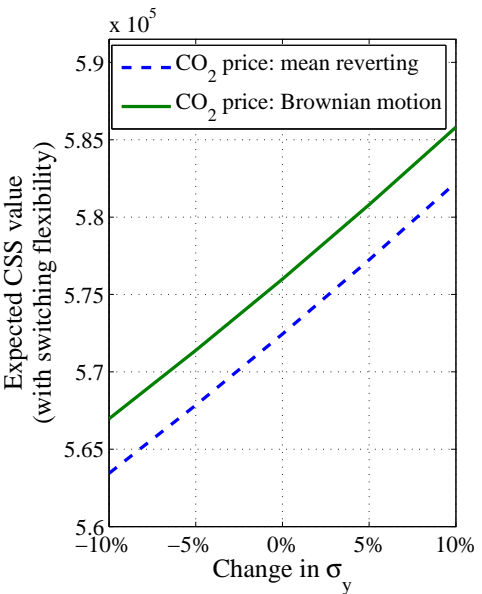

(b)

Figure 4.12: Expected clean spark spread with respect to $\mathrm{CO}_{2}$ price processes: (a) without flexibility, and (b) with flexibility. 

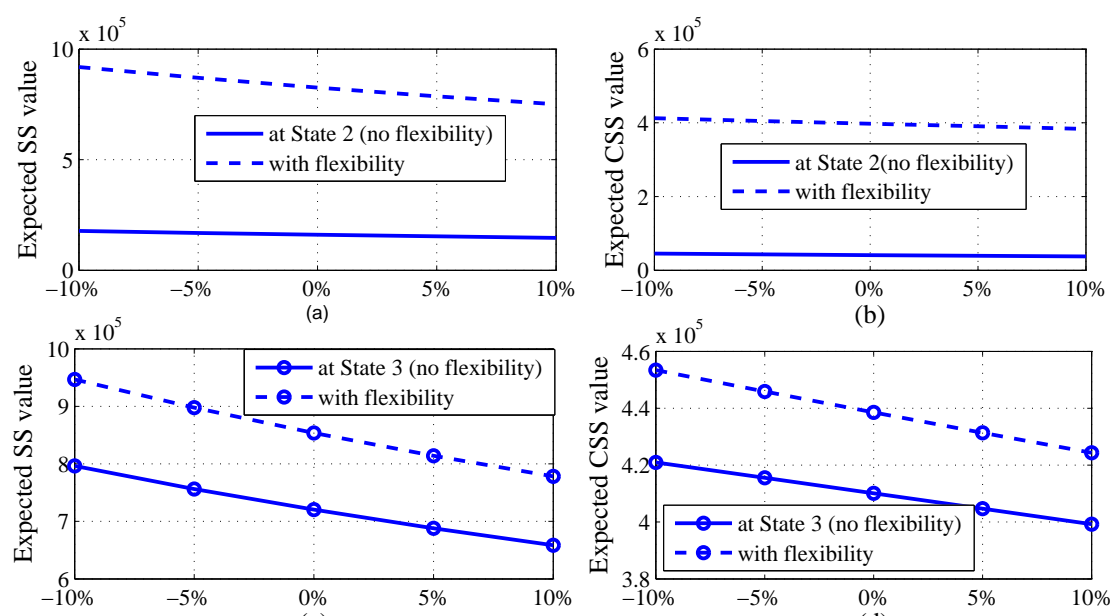

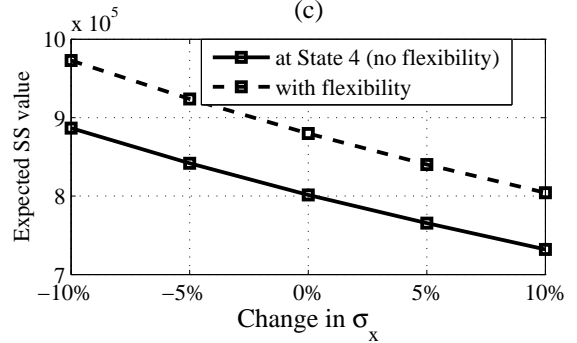

(e)

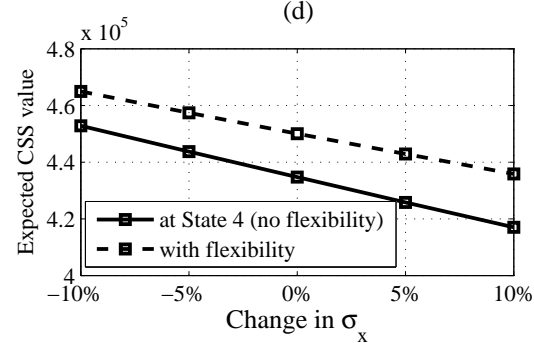

(f)

Figure 4.13: Expected spark and clean spark spread values of the power plant due to changes in the natural gas price volatility: (a) State 2 (without carbon cost), (b) State 2 (with carbon cost), (c) State 3 (without carbon cost), (d) State 3 (with carbon cost), (e) State 4 (without carbon cost), and (f) State 4 (with carbon cost). 

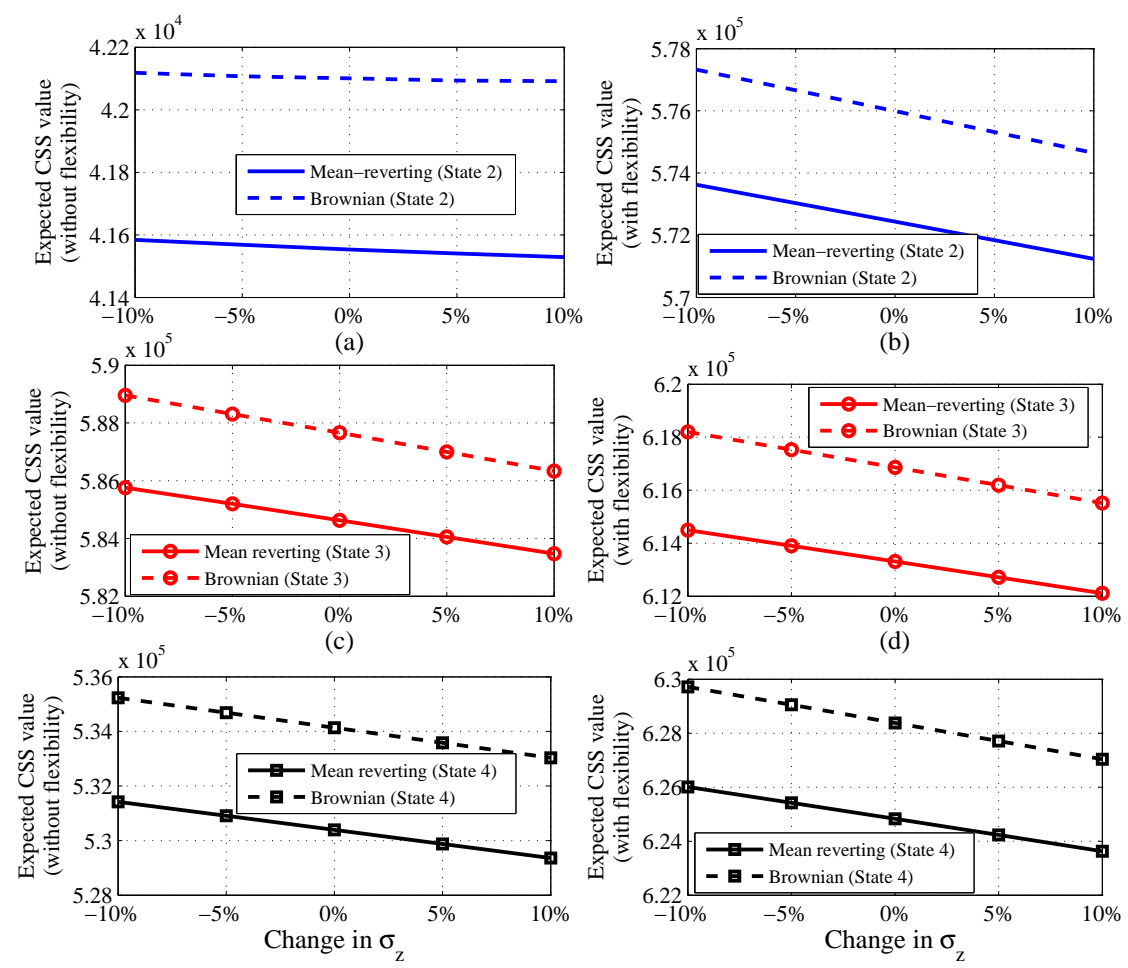

(e)

(f)

Figure 4.14: Expected clean spark spread value with respect to changes in the $\mathrm{CO}_{2}$ price volatility: (a) State 2 (without flexibility), (b) State 2 (with flexibility), (c) State 3 (without flexibility), (d) State 3 (with flexibility), (e) State 4 (without flexibility), and (f) State 4 (with flexibility). 


\subsubsection{Sensitivity to the mean-reverting speed of price}

A higher mean-reverting speed tends to stabilize the price movement towards the long term mean quickly. Therefore, the expected spark and clean spark spread option values of the power plant decrease as the mean-reverting speed of electricity price increases. This is evident from Figure 4.15 that an increasing mean-reverting speed of electricity price decreases the expected value of the power plant.
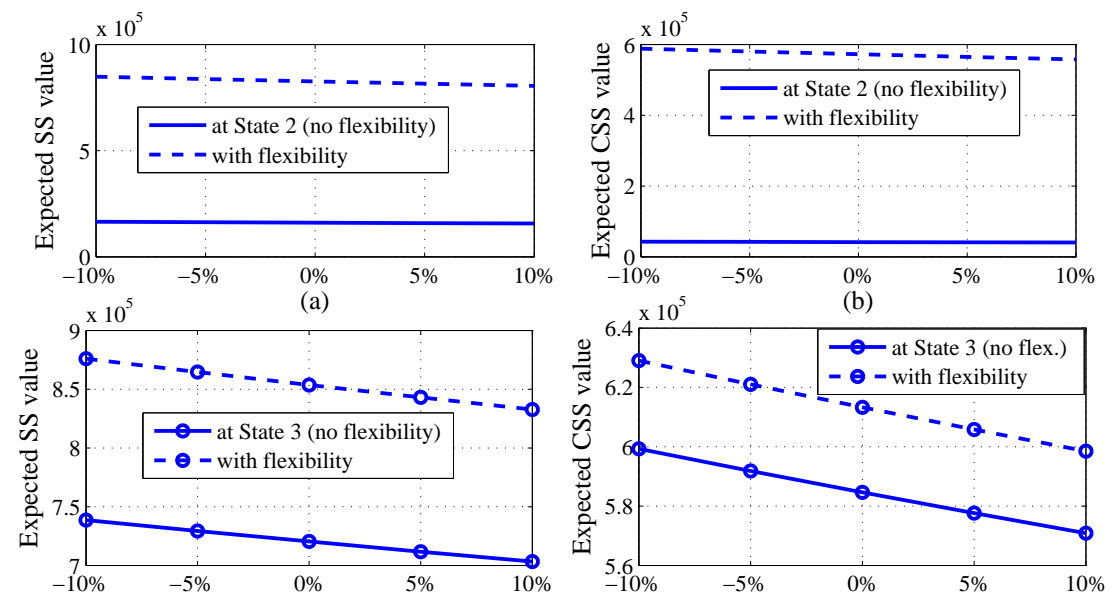

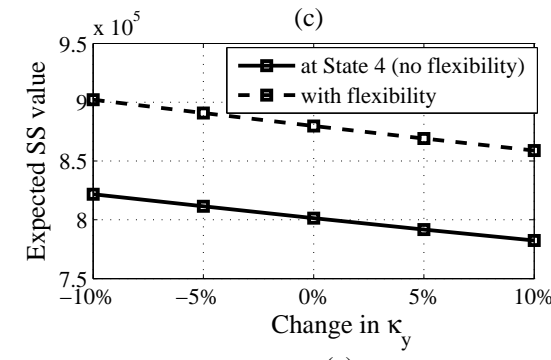

(e)

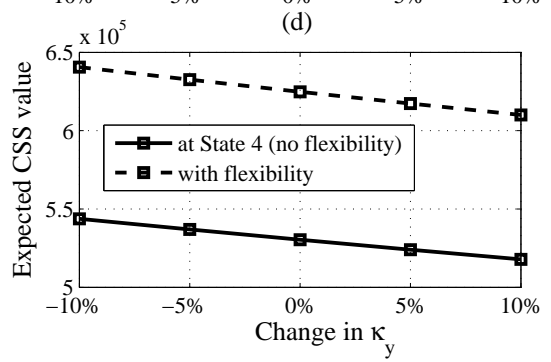

(f)

Figure 4.15: Expected spark and clean spark spread values due to changes in the mean-reverting speed of electricity price: (a) State 2 (without carbon cost), (b) State 2 (with carbon cost), (c) State 3 (without carbon cost), (d) State 3 (with carbon cost), (e) State 4 (without carbon cost), and (f) State 4 (with carbon cost).

Similarly, an increase in the mean-reverting speed of natural gas price tends to pull back the price quickly to the long-term mean price that results in smaller changes in the price over time. Since natural gas price is in the form of cost, the expected spark and clean spark spread option values of the power plant increase (see Figure 4.16) with an increase of the mean-reverting speed of natural gas price. 

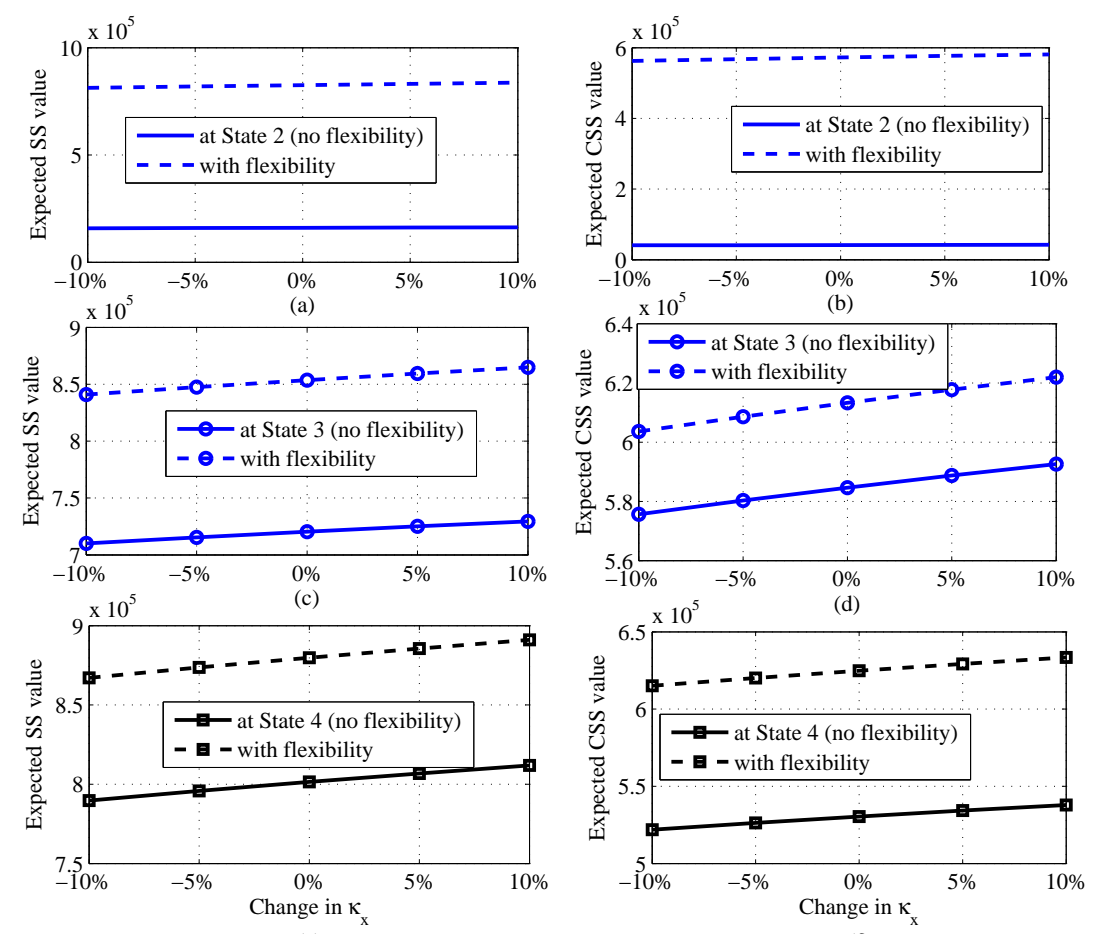

(e)

(f)

Figure 4.16: Expected spark and clean spark spread values due to changes in the mean-reverting speed of natural gas price: (a) State 2 (without carbon cost), (b) State 2 (with carbon cost), (c) State 3 (without carbon cost), (d) State 3 (with carbon cost), (e) State 4 (without carbon cost), and (f) State 4 (with carbon cost). 


\subsubsection{Sensitivity to changes in the minimum capacities of turbines}

A gas turbine's minimum capacity is usually $10 \%$ of its maximum capacity. Figure 4.17 shows the expected values of the power plant based on spark spread and clean spark spread options, if both Turbine 1 and Turbine 2's minimum capacities vary simultaneously from 5 MW to $50 \mathrm{MW}$. It is observed that the expected values are not sensitive to the changes in the minimum capacities of turbines. This is because when price movements are favorable, thus the spark spread is positive, the power plant is operated at the maximum possible capacitates. When the spark spread is not favorable, the operator does not run the power plant at all. The operation of the power plant here is similar to a base load type power plant, which operates at its maximum capacities most of the time.
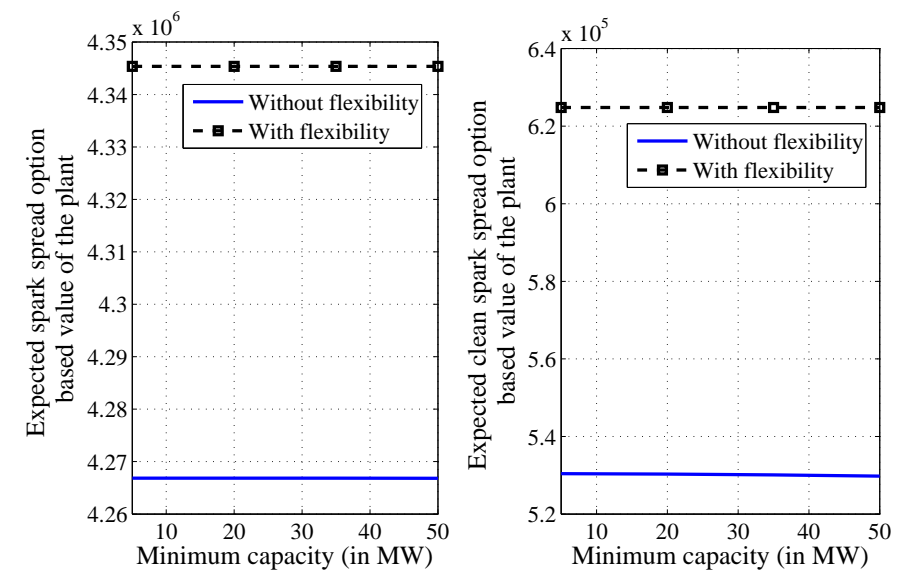

Figure 4.17: Expected spark and clean spark spread values with respect to the changes in the minimum capacities of turbines. 


\subsection{Summary}

This chapter analyzes spark and clean spark spread option based valuation of a merchant power plant with multiple natural gas-fired turbines. How operational flexibility of switching in accordance with the correlated price movements affect the valuation of a power plant under environmental restrictions, particularly in presence and absence of carbon allowance price, is investigated. Electricity and natural gas prices are assumed to follow mean-reverting processes. In the clean spark spread option based valuation, two cases are considered. In one case, $\mathrm{CO}_{2}$ allowance price is assumed to follow a mean-reverting process; in another scenario, $\mathrm{CO}_{2}$ price is assumed to follow a geometric Brownian motion. A comparison of these two cases is also presented. Results show that presence of $\mathrm{CO}_{2}$ allowance cost reduces the value of the power plant, while operational flexibility of switching among turbines in accordance with price movements adds value to the power plant. It is also observed that for a power plant with two gas turbines, in presence of $\mathrm{CO}_{2}$ allowance cost, operating an efficient gas turbine with a lower carbon emission rate may offer a higher expected value than that of operating both turbines. Sensitivity tests with respect to electricity price volatilities show that both spark and clean spark spread option based value of the power plant increases with an increase in the electricity price volatility. However, an increase in the volatilities of natural gas and $\mathrm{CO}_{2}$ prices reduces the expected power plant value. Sensitivity tests with respect to the mean-reverting speed of prices show that an increase in the mean-reverting speed of electricity price reduces the value of the power plant, whereas an increase in the mean-reverting speed of natural price tends to increase the value of the power plant. In case of clean spark spread option based valuation, it is found that modeling $\mathrm{CO}_{2}$ price as a Brownian motion overvalues the plant in comparison to when $\mathrm{CO}_{2}$ allowance price is assumed to follow a mean-reverting process. In the next chapter, the valuation model of a power plant with multiple turbines integrating spark spread and weather options is discussed. 


\section{Chapter 5}

\section{Integrating Weather Options in the}

\section{Valuation of a Power Plant with}

\section{Multiple Turbines}

\subsection{Introduction}

Whims of weather also affect operations of a power plant. During a cold winter, an increased heating requirement would induce an increased power demand from the power plant, which in turn, could produce a higher payoff. However, if the winter is not-so-cold or mild, a lower heating requirement could decrease the payoff. One of the solutions is to purchase daily weather options. Through weather derivatives, a power plant can transfer its weather related uncertainties to a financial firm willing to absorb the risk. This risk is contingent on a predetermined underlying weather variable, for example, heating degree days (HDD) measured by summing up temperature degrees below a base temperature, for example, $18^{\circ} \mathrm{C}$.

\subsection{Integrating spark spread and weather options}

For a power plant having $G$ turbines, the plant can be at any of $2^{G}$ states. Let $\mathbf{s}$ denote the possible states of the plant defined by the different combinations of 'on/off' status of turbines. For example, a power plant having two turbines can have four states denoted by $\mathbf{s}$, such that $\mathbf{s}=\left(\begin{array}{llll}s_{1} & s_{2} & s_{3} & s_{4}\end{array}\right)^{\prime}$, where $s_{1}=\left[\begin{array}{ll}0 & 0\end{array}\right]$ denotes that both Turbine 1 and Turbine 2 are at the 'off' state; $s_{2}=\left[\begin{array}{ll}1 & 0\end{array}\right]$ represents that Turbine 1 is 'on' but Turbine 2 is 'off'; and in similar fashion, $s_{3}=\left[\begin{array}{ll}0 & 1\end{array}\right]$, and $s_{4}=\left[\begin{array}{ll}1 & 1\end{array}\right]$. The prime notation refers to the transposition of a matrix. Therefore, $\mathbf{s}$ is a $4 \times 2$ matrix as $\mathbf{s}=\left(\begin{array}{lllll}0 & 0 ; 1 & 0 ; 0 & 1 ; 1 & 1\end{array}\right)^{\prime}$. The flexibility of switching, in this study, is defined as the 
ability of switching from one state to another state, for example, from $s_{2}$ to $s_{3}$ or from $s_{4}$ to $s_{3}$, depending on the movement of prices and temperature.

For a gas turbine, $g$, generating $q_{g} \mathrm{MW}$ of electricity, let $H\left(q_{g}\right)$ be the fuel input in MMBTu/hr. $H\left(q_{g}\right)$ can be expressed as an input-output characteristic relationship as (Wood and Wollenberg 1996; Tseng et al. 2009):

$$
H\left(q_{g}\right)=\zeta\left(Q_{g}^{e}\right)\left(a_{g}+b_{g} q_{g}+c_{g} q_{g}^{2}\right)
$$

where, coefficients, $a_{g}, b_{g}$, and $c_{g}$ are nonnegatives. The noload cost is represented by $a_{g}>0$, $b_{g}>0$ implies the incremental heat rate and $c_{g}>0$ indicates that the cost function is convex. The notion of $\zeta\left(Q_{g}^{e}\right)$ is to incorporate the effect of environment temperature on the gas turbine power generation, which in turn, affects the value of the spark spread option. A gas turbine performs better in a cooler environment (Tseng et al. 2009). The adjusting factor, $\zeta\left(Q_{g}^{e}\right)$, therefore, can be expressed as:

$$
\zeta\left(Q_{g}^{e}\right)=1+\left(\frac{Q^{e}-Q_{g}^{d}}{\Delta Q_{g}^{d}}\right) R^{c},
$$

where, $Q^{e}$ is the environment temperature, $Q_{g}^{d}$ is the design temperature for Turbine $g$, and $\Delta Q_{g}^{d}$ is the maximum allowable deviation of operating temperature from the design temperature for Turbine $g$. When environment temperature, $Q^{e}$, is equal to $Q_{g}^{d}$, the adjusting factor, $\zeta\left(Q_{g}^{e}\right)$ is equal to 1 . If $Q^{e}$ is different from $Q_{g}^{d}$, the adjusting factor, $\zeta\left(Q_{g}^{e}\right)$, varies linearly. $R^{c}$ is an empirical parameter, between 0 and 1 , representing a measure in the cost function when environment temperature deviates from the design temperature. The roles of $\zeta\left(Q_{g}^{e}\right)$ and $R^{c}$ along with changes in electricity and natural gas prices in determining the feasible optimal power generation from a turbine are illustrated in Section 5.2.1.

\subsubsection{The spark spread option}

The power plant owner buys the natural gas at a price of $P_{n}$ in $\$ / \mathrm{MMBTu}$, burns and converts it into electric power and sells the electricity at a price of $P_{e}$ in $\$ / \mathrm{MWh}$. The plant earns a profit (in $\$ /$ hr) by running each turbine from the spark spread:

$$
\begin{aligned}
& \Omega_{g}=q_{g} P_{e}-H\left(q_{g}\right) P_{n}-q_{g} v_{g} \\
& \Omega_{g}=q_{g} P_{e}-\zeta\left(Q_{g}^{e}\right)\left(a_{g}+b_{g} q_{g}+c_{g} q_{g}{ }^{2}\right) P_{n}-q_{g} v_{g},
\end{aligned}
$$

where $v_{g}$ is the operation and maintenance cost per MWh and $H\left(q_{g}\right)$ is the fuel input in MMBTu/hr for generating $q_{g} \mathrm{MW}$ of electricity. The owner earns a profit, if the revenue from selling electricity is more than the cost of burning natural gas and the cost of operation and maintenance. 
The optimal quantity of generation, $q_{g}^{*}$, therefore, can be obtained by differentiating Equation (5.3) with respect to $q_{g}$ and setting it to zero. The optimal generation $q_{g}^{*}$ is obtained as:

$$
q_{g}^{*}=\frac{1}{2 c_{g} \zeta\left(Q_{g}^{e}\right)}\left(\frac{P_{e}-v_{g}}{P_{n}}-\zeta\left(Q_{g}^{e}\right) b_{g}\right)
$$

Since a gas turbine has the minimum and maximum operating capacities of $\underline{q}_{g}$ and $\bar{q}_{g}$ respectively, the optimal feasible operating quantity, $\hat{q}_{g}$ becomes:

$$
\hat{q}_{g}=\max \left(\underline{q}_{g}, \min \left(q_{g}^{*}, \bar{q}_{g}\right)\right) .
$$

Figure 5.1 demonstrates how $\zeta\left(Q_{g}^{e}\right)$ and $R^{c}$ along with the changes in prices affect the adjusting factor, $\hat{q}_{g}$, and the optimal feasible generation from a turbine, $\hat{q}_{g}$, obtained by using Equation (5.4). It is observed that $\zeta\left(Q_{g}^{e}\right)$ varies with the environment temperature at different $R^{c}$. A higher value of $R^{c}$ induces a higher feasible power generation when the environment temperature is below $18^{0} \mathrm{C}$. As the temperature moves above $18^{0} \mathrm{C}$, a higher $R^{c}$ value causes a lower power generation. The equation thus captures the fact that "for every K (Kelvin) rise in ambient temperature above ISO (International Organization for Standardization) conditions the gas turbine loses" its thermal efficiency and useful power output (Sa and Zubaidy 2011). Another observation from Figure 5.1 shows that a higher electricity price also prompts the power plant to generate more power.
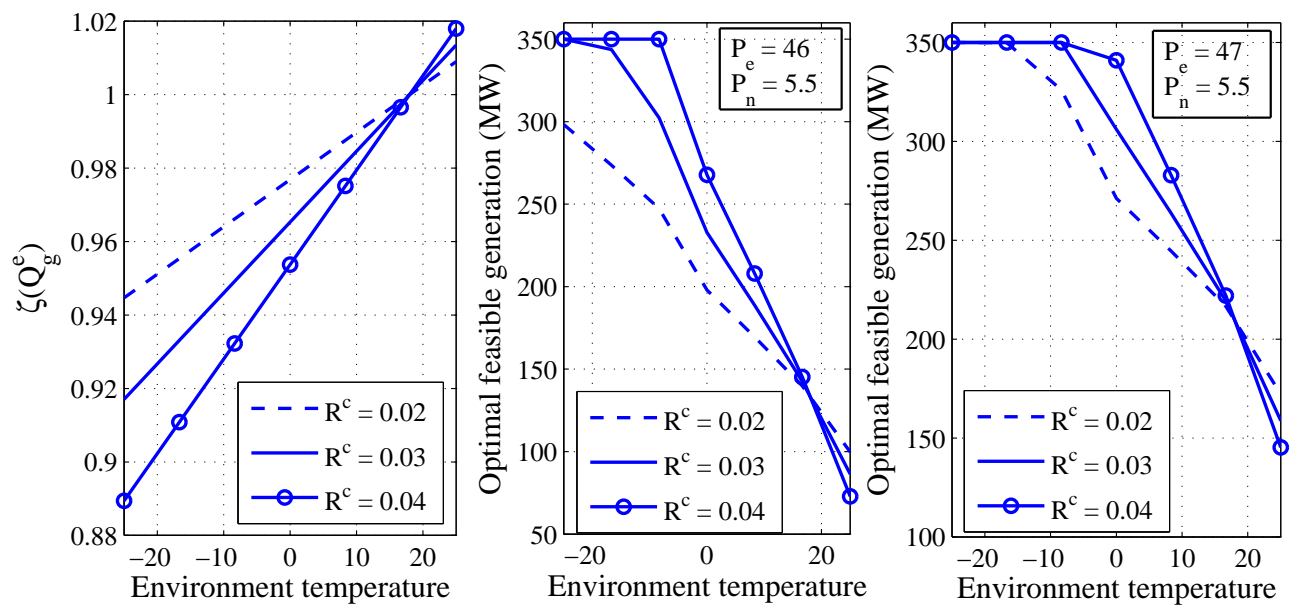

Figure 5.1: The variations of the adjusting factor, $\zeta\left(Q_{g}^{e}\right)$, and the optimal feasible operating quantity, $\hat{q}_{g}$, with respect to environment temperature and prices at different $R^{c}$.

The value of the spark spread, $\Omega_{g}$, from each turbine of a power plant operating $h$ hours per day, 
therefore, can be expressed by replacing $q_{g}$ in Equation (5.3) by $\hat{q_{g}}$ as:

$$
\Omega_{g}=h\left\{\hat{q}_{g} P_{e}-\zeta\left(Q^{e}\right)\left(a_{g}+b_{g} \hat{q}_{g}+c_{g} \hat{q}_{g}^{2}\right) P_{n}-\hat{q}_{g} v_{g}\right\}
$$

The value of spark spread at different states, s, of the power plant, can be expressed as:

$$
\boldsymbol{\Pi}_{s}=\mathbf{s} \cdot\left(\boldsymbol{\Omega}_{g}\right)^{\prime}
$$

As an example, for a power plant with two turbines, the values of the spark spread at different states can be expressed as:

$$
\boldsymbol{\Pi}_{s}=\left(\begin{array}{llll}
0 & 1 & 0 & 1 \\
0 & 0 & 1 & 1
\end{array}\right)^{\prime} \cdot\left(\begin{array}{l}
\Omega_{1} \\
\Omega_{2}
\end{array}\right)
$$

The value of the spark spread options, $\pi_{s}$, therefore, can be expressed as:

$$
\pi_{s}=\max \left(0, \boldsymbol{\Pi}_{s}\right)
$$

\subsubsection{The weather option}

It is assumed that the plant owner, depending on the state of the plant, $\mathbf{s}$, longs $n_{\mathbf{s}}$ number of weather put options for a premium of $\varphi$. If weather conditions behave as usual and the winter is cold, the put option remains unexercised. However, if the winter is warm and the accumulated HDD drops below the strike HDD, the plant owner exercises the option and earns a payoff from exercising the option.

As shown in Figure 5.2, assume that the plant owner buy option from day $t=1$ until day $t=T-t_{c}+1$, where $t_{c}$ is the contract period over which HDD is accumulated. For example, for a planning period from day $t=1$ to day $T=15$, i.e., $[1,15]$, the plant owner buys weekly weather options on day $t=1$ and exercises it on day $t=t_{c}=7$ contingent on the accumulation of HDD from day 1 to day 7 . He/she then buys options on day $t=2$ and exercises it on day $t=t_{c}+1=7+1=8$; and so on. Since weather option exercise is matured at $t=t_{c}$, as seen from Figure 5.2, for the period from $t=t_{c}$ to $t=T$, the payoffs come from both spark spread and weather options; while for the period from $t=1$ to $t=t_{c}-1$, the payoff is contributed by the spark spread option only.

The profit, $\omega$, from the weather option is realized from day $t=t_{c}$ to $t=T$ and is given by:

$$
\omega=\tau \max (0, K-\overline{\mathrm{HDD}})-\varphi e^{r\left(t_{c} / 250\right)} \text { for } t_{c} \leq t \leq T
$$

where $\tau$ is tick size or payment per HDD, $K$ is the strike HDD, $t_{c}$ is the weather derivative contract 
period, e.g., a weekly contract period, $\varphi$ is the option premium and $\overline{\mathrm{HDD}}$ refers to the average of path dependent HDD accumulated over the contract period. As the degree-days derivative is alike an Asian type option (Geman 2000), the mean of the path dependent HDD over the contract time is taken to calculate the weather payoff.

\subsubsection{Pricing weather derivatives}

The pricing of temperature-based weather derivatives depends on the appropriate modeling of the stochastic behavior of temperature. Dorfleitner and Wimmer (2010) mention that "there is no general consensus regarding the question concerning how to price weather derivatives". In the literature, pricing mechanisms of weather derivatives range from the marginal utility technique (e.g., Davis 2001) to the simulation approach based on the Ornstein-Uhlenbeck process (e.g., Alaton et al. 2002) to an autoregressive temperature modeling approach (e.g., Benth et al. 2007). In this chapter, Nelson and Ramaswamy (1990) binomial model is utilized to discretize the mean-reverting behavior of temperature. The daily mean temperature $Q_{t}$ can be modeled as, $Q_{t}=w_{t}+S_{t}$, where $S_{t}$ is the seasonal component and $w_{t}$ is the deseasonalized stochastic component of the temperature, which is assumed to follow a mean-reverting process (Benth et al. 2007). The step size is defined by $\sigma_{w} \sqrt{d t}$. At any node, $w_{t}$ may jump up or down with the corresponding branch probabilities given by $\xi=\min \left[1, \max \left(0, \frac{1}{2}+\kappa_{z}\left(\bar{w}-w_{t}\right) \sqrt{d t} / 2 \sigma_{w}\right)\right]$ and $(1-\xi)$, respectively.

In order to retrieve the temperature, $Q_{t}$, the deterministic annual seasonal component, $S_{t}$, is added to the value of $w_{t}$ at each node of the lattice. A recursive function is applied to calculate the expected HDD as follows:

$$
\operatorname{HDD}_{t}=\max \left(0, \bar{Q}-Q_{t}\right)+\mathbf{E}\left[\operatorname{HDD}_{t+1}\right]
$$

where, $\bar{Q}$ is the base temperature, $18^{0} \mathrm{C}$, and $Q_{t}$ is the daily mean temperature. The option price,

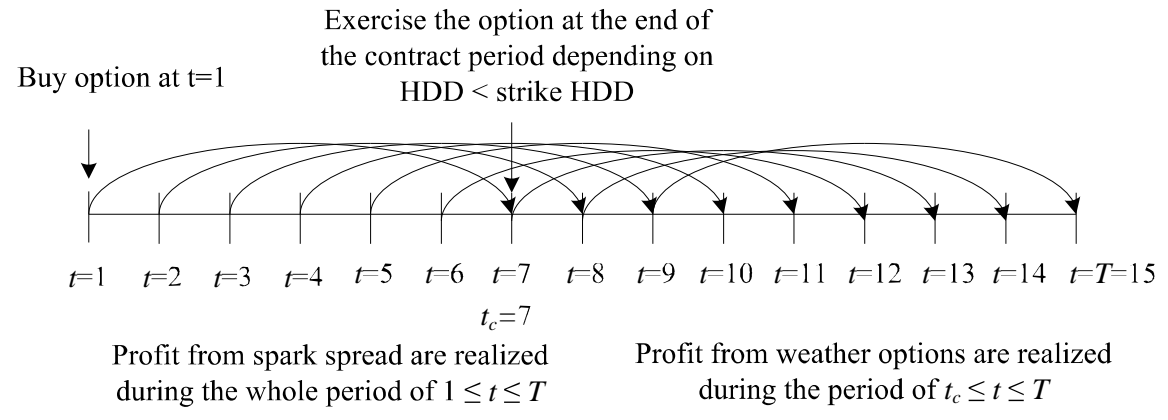

Figure 5.2: The exercise of weather options. 
$\varphi$, is calculated as:

$$
\varphi=\tau \times \max (0, K-\mathrm{HDD}) e^{-r t_{c}}
$$

where, $\tau$ is the tick size or the payment per HDD, $K$ is the strike heating degree days, $t_{c}$ is the contract period, and $r$ is the risk free discount rate.

\subsubsection{Number of contracts}

The total profit of the plant is the summation of payoffs from the spark spread option, $\pi$, and weather options, $\omega$ :

$$
\Theta=\pi+n \omega
$$

where $n(n \geq 0)$ is the number of weather contracts to buy. Since an individual power plant owner is interested only to reduce the variance of her own income relative to the variance of market prices (McKinnon 1967), the variance minimization of the total payoff, $\Theta$, yields the optimal number of weather contracts to buy. Therefore, from Equation (5.13), the variance, $\mathbf{V}(\Theta)$, can be defined as:

$$
\begin{aligned}
& \mathbf{V}(\Theta)=\mathbf{V}(\pi)+n^{2} \mathbf{V}(\omega)+2 n \gamma \sqrt{\mathbf{V}(\pi) \mathbf{V}(\omega)} \\
& \mathbf{V}(\Theta)=\sigma_{\pi}^{2}+n^{2} \sigma_{\omega}^{2}+2 n \gamma \sigma_{\pi} \sigma_{\omega}
\end{aligned}
$$

where $\gamma$ is the correlation coefficient between the payoff from the spark spread option and the payoff from the weather option. Setting $\frac{\partial \mathbf{V}(\Theta)}{\partial n}=0$, the number of contracts, $n$, is as follows:

$$
\begin{aligned}
\left(2 n \sigma_{\omega}^{2}+2 \gamma \sigma_{\pi} \sigma_{\omega}\right) & =0 \\
n & =-\gamma \frac{\sigma_{\pi}}{\sigma_{\omega}}
\end{aligned}
$$

Equation (5.15) implies that the number of weather contract is dependent on the ratio of the volatilities of spark spread and weather option payoffs, and the correlation coefficient between the payoff from spark spread and weather options. The implication of the negative sign in Equation (5.15) is that while a lower HDD in a warmer winter would cause a reduced spark spread payoff, $\pi$, it (i.e., the lower HDD) would incur a higher weather put option payoff, $\omega$.

Since options are bought continuously from day $t=1$ until day $t=T-t_{c}+1$ (see Figure 5.2), the number of weather contracts, $n_{\mathbf{s}_{t}}$, depending on the state of the plant, can be written as:

$$
n_{\mathbf{s}_{t}}=-\gamma_{\left\{t+t_{c}-1\right\}} \frac{\sigma_{\pi_{\mathbf{s}}\left\{t+t_{c}-1\right\}}}{\sigma_{\omega_{\left\{t+t_{c}-1\right\}}}} \text { for } 1 \leq t \leq T-t_{c}+1 .
$$


The time subscript indicates decisions over the time. For example, at day $t=1$, the decision to buy the number of contracts depends on the value of the correlation coefficient and the volatilities of spark spread and weather payoff by day 7 . Similarly at day $t=2$, the decision to buy the number of contracts depends on the correlation coefficient and volatility parameters by day 8 .

\subsubsection{The value of the power plant}

Payoff from spark spread option is realized at each interval of the lattice. Since a weather option is matured after a contract period, $t_{c}$, the weather option payoff is not realized during the period from $t=1$ to $t=t_{c}-1$ and the total payoff is obtained from the spark spread option only. Therefore, the combination of payoffs from the spark spread and weather options, $\Theta$, can be expressed as:

$$
\begin{array}{ll}
\Theta_{t}\left(\pi_{\mathbf{s}}, \omega\right)=\pi_{\mathbf{s}_{t}} & \text { for } 1 \leq t \leq t_{c}-1, \quad(\text { Since } \omega=0) \\
\Theta_{t}\left(\pi_{\mathbf{s}}, \omega\right)=\pi_{\mathbf{s}_{t}}+n_{\mathbf{s}_{\left\{t-t_{c}+1\right\}}} \omega_{t} & \text { for } t_{c} \leq t \leq T
\end{array}
$$

The value of the plant is then determined as a summation of the current total profit plus the discounted expected total profit from the next period by using a backward dynamic programming approach. Therefore, the value of the plant, $\mathbf{W}_{t}$, can be expressed by using a recursive function as follows:

$$
\mathbf{W}_{t}=\Theta_{t}\left(\pi_{\mathbf{s}}, \omega\right)+e^{-r(\Delta t)} \mathbf{E}_{t}\left[\mathbf{W}_{t+1}\right]
$$

The value of the power plant with the switching flexibility, $\mathbf{F}_{t}$, can be expressed as:

$$
\mathbf{F}_{t}=\Theta_{t}\left(\pi_{\mathbf{s}}, \omega\right)+e^{-r(\Delta t)} \max \left\{\mathbf{E}_{t}\left[\mathbf{F}_{t+1}\right]+C\left((s)_{t, t+1}\right)\right\},
$$

where $C\left((s)_{t, t+1}\right)$ is the switching cost from one state to another state of the power plant. For example, if startup and shutdown costs for gas turbine 1 are $C_{u 1}$ and $C_{d 1}$, respectively; and for turbine 2 are $C_{u 2}$ and $C_{d 2}$, respectively, then $C\left((s)_{t, t+1}\right)$ is a $4 \times 4$ matrix as:

$$
C\left((s)_{t, t+1}\right)=\left(\begin{array}{cccc}
0 & C_{u 1} & C_{u 2} & C_{u 1}+C_{u 2} \\
C_{d 1} & 0 & C_{d 1}+C_{u 2} & C_{u 2} \\
C_{d 2} & C_{u 1}+C_{d 2} & 0 & C_{u 1} \\
C_{d 1}+C_{d 2} & C_{d 2} & C_{d 1} & 0
\end{array}\right)
$$

Operating constraints such as, startup, shutdown, ramp time and costs, minimum up and down time, are usually measured hourly (Johnson and Miranda 2003). However, in the above cost matrix, the switching costs are assumed to represent average daily costs taking into account all costs related 
to hourly operating constraints for a turbine. Since HDD is calculated on a daily basis, in order to integrate weather option with spark spread option, a daily time-interval is chosen for the proposed model.

\subsection{Mean-reverting processes}

For the valuation of a natural gas-fired power plant, a correlated movement of prices of electricity, natural gas and corresponding temperature is needed. As stated in Section 2.6 and Section 3.2, temperature dynamics and commodity prices are usually modeled by mean-reverting processes in the literature, accordingly it is assumed that the prices of electricity and natural gas and temperature follow mean-reverting processes in this chapter. Figure 5.3 shows the futures prices of electricity and natural gas taken from European Energy Exchange (EEX) for the period of January, 2009 to December, 2010. The prices are converted to Canadian dollar (CAD) by using corresponding daily exchange rates from Euro to CAD. The corresponding exchange rates are collected from the historical data of the Bank of Canada so that these prices can be used as proxy prices for Canada. Figure 5.4 shows the corresponding daily mean temperature for the city of Toronto, Canada, taken from the website of Environment Canada.
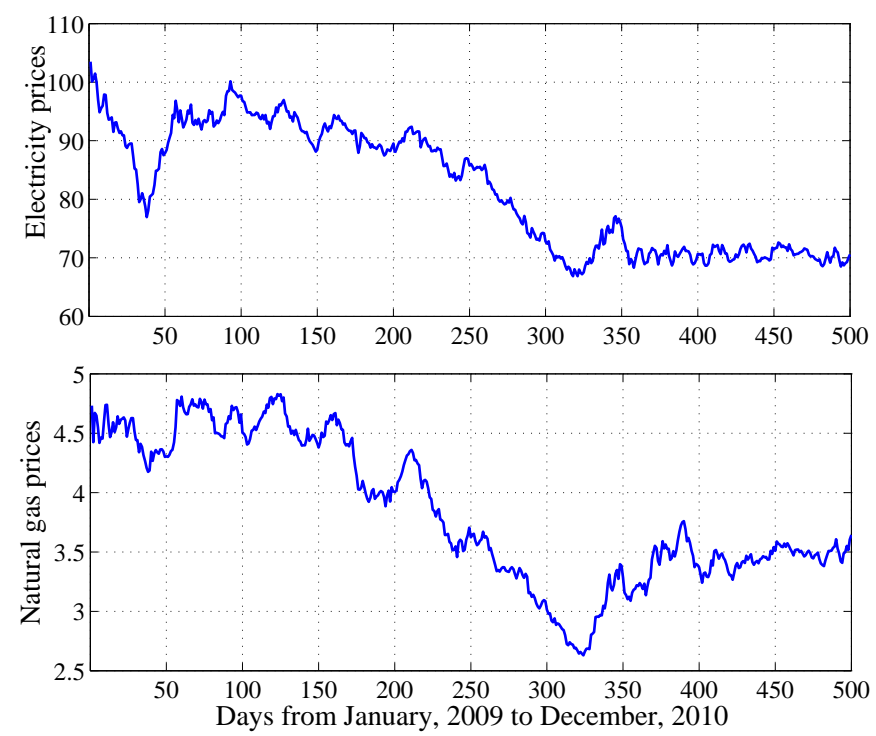

Figure 5.3: Futures prices of electricity in $\mathrm{CAD} / \mathrm{MWh}$ (top) and natural gas futures prices in CAD/MMBtu (bottom) from European Energy Exchange (EEX) scaled to Canadian dollar using corresponding daily exchange rates from Euro to CAD. 

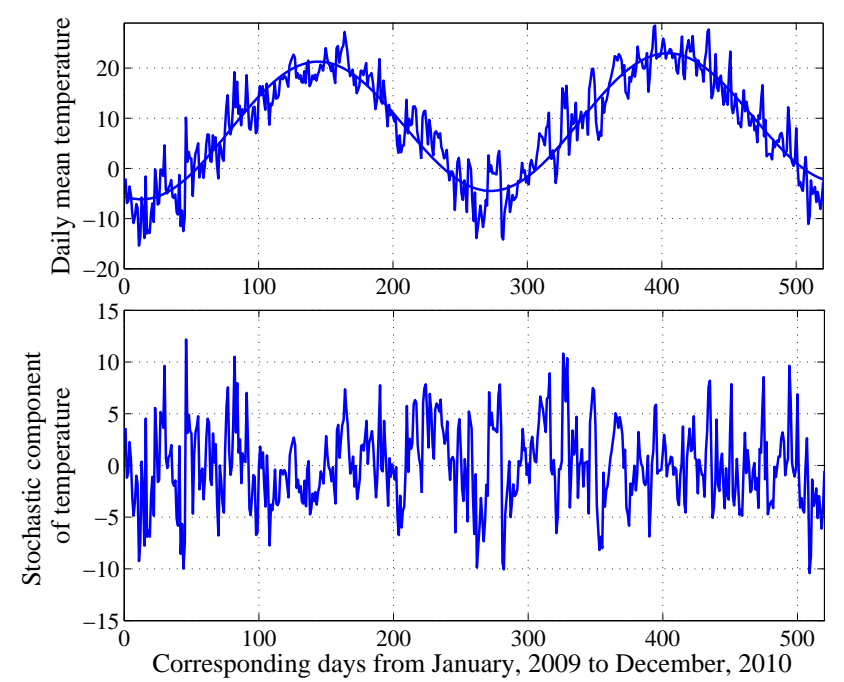

Figure 5.4: Corresponding daily mean temperature for the city of Toronto, Canada.

\subsubsection{An estimation of mean-reverting process parameters}

In order to estimate the process parameters, mean, speed of reversion and volatility, the meanreverting process is discretized into the first order auto-regression, $\operatorname{AR}(1)$, process (see Appendix D). For example, in the case of the natural gas price process, the discretization can be expressed as: $\frac{\Delta x_{t}}{\Delta t}=\alpha+\varrho x_{t}+\epsilon_{t}$, where $\alpha$ is the intercept, $\varrho$ is the slope of the linear regression line drawn by minimizing the sum of the squared residuals, and $\epsilon_{t} \sim \mathrm{N}\left(0, \sigma_{x}^{2}\right)$ are the residuals. These coefficients of the discretized mean-reverting processes as respective $\operatorname{AR}(1)$ processes are shown in Table 5.1. From Table 5.1, parameters obtained for the natural gas price process are: $\kappa_{x}=|\varrho|=1.6218$,

Table 5.1: Mean-reverting parameters for price processes

\begin{tabular}{lcrrrr}
\hline Prices & Coeff. & Value & Std. err. & t-stats & p-value \\
\hline Natural gas & $\alpha$ & 2.0267 & 1.4266 & 1.4207 & 0.1560 \\
& $\varrho$ & -1.6218 & 1.0643 & -1.5238 & 0.1282 \\
\hline Electricity & $\alpha$ & 7.3824 & 4.5049 & 1.6387 & 0.1019 \\
& $\varrho$ & -1.7249 & 1.0255 & -1.6820 & 0.0932 \\
\hline Temperature & $\alpha$ & -1.8875 & 35.2917 & -0.0535 & 0.9574 \\
& $\varrho$ & -110.6080 & 9.1162 & -12.1331 & 0.0000 \\
\hline
\end{tabular}

$\bar{x}=\frac{\alpha}{\varrho \varrho \mid}=1.2497, \sigma_{x}=\operatorname{std}($ residuals $) \times \sqrt{\Delta t}=0.23536$, where 'std' stands for the standard deviation. Similarly, for the electricity price process, $\bar{y}=4.28, \kappa_{y}=1.7249, \sigma_{y}=0.18568$; and for the temperature process, $\bar{w}=-0.017065, \kappa_{w}=110.6080, \sigma_{w}=50.8004$. Correlation coefficients of respective price processes are: $\rho_{x y}=0.5397, \rho_{y w}=0.0250$, and $\rho_{w x}=-0.0274$. 


\subsection{A numerical example}

This section provides an example of the lattice based approach in details. Section 5.4.1 discusses the construction of the lattice. Sections 5.4.2 and 5.4.3 show how to calculate the number of weather contracts and the weather option payoff, respectively.

\subsubsection{Lattice construction}

A trivariate lattice is built according to the outline discussed in Section 3.3.2. Step sizes are determined using Equations (3.4), (3.5), and (3.22). Following these step sizes, movements of the logarithm values of natural gas prices, electricity prices and the stochastic component of temperature are calculated at each node and at each time interval of the lattice. Corresponding branch probabilities are calculated using Equations (3.31) - (3.38). Exponentials are taken to retrieve the values of prices of natural gas and electricity. Seasonal part of temperature is added to the corresponding stochastic component of temperature to retrieve the values of temperature at each node and at each time interval. The values of spark spread option, number of contracts, and total payoff are calculated at different states of the power plant. A recursive backward dynamic programming approach is then applied to determine the value of the plant.

With initial values of $x_{1}=\ln (4.0), y_{1}=\ln (70.0)$, and $w_{1}=8.5570$, the step sizes are calculated using Equations $(3.4),(3.5)$, and $(3.22)$, as: $0.0149(=0.2354 \times \sqrt{1 / 250}), 0.0117(=0.1857 \times \sqrt{1 / 250})$ and $3.2129(=50.8004 \times \sqrt{1 / 250})$, respectively. In the next step, the exponentials of $x_{t}$ and $y_{t}$ are taken to retrieve the values of the natural gas price and electricity price, respectively, and the sinusoidal seasonal component of temperature, $S_{t}$, is added to $w_{t}$ to obtain the values of temperature. Adding the seasonal component, $S_{1}=-5.6338$ to $w_{1}=8.5570$, the initial temperature is obtained as: $2.9232(=8.5570-5.6338)$. Branch probabilities are calculated using Equations (3.31)-(3.38). For example, between the layers at $t=1$ and at $t=2, p_{u u u}^{*}=0.0, p_{u u d}^{*}=0.3713, p_{u d u}^{*}=0.0$, $p_{u d d}^{*}=0.0952, p_{d u u}^{*}=0.0, p_{d u d}^{*}=0.1350, p_{d d u}^{*}=0.3985$, and $p_{d d d}^{*}=0.0$. The payoff from spark spread options at each state of the plant at each node is calculated by using Equation (5.9) and the payoff from weather option is calculated by using Equation (5.10). Figure 5.5 (top) shows the movements of natural gas and electricity prices and temperature and Figure 5.5 (bottom) shows the values of the spark spread options at different states along with the accumulated HDD.

\subsubsection{Number of contracts}

For exercising a weekly weather contract, if an option is bought at day $t=1$, it is exercised at day $t=7$. Figure 5.6 shows a two dimensional projection of spark spread option payoff from the lattice at State 2 of the power plant at $t=7$. Figure 5.7 shows the weather option payoff at $t=7$. The 

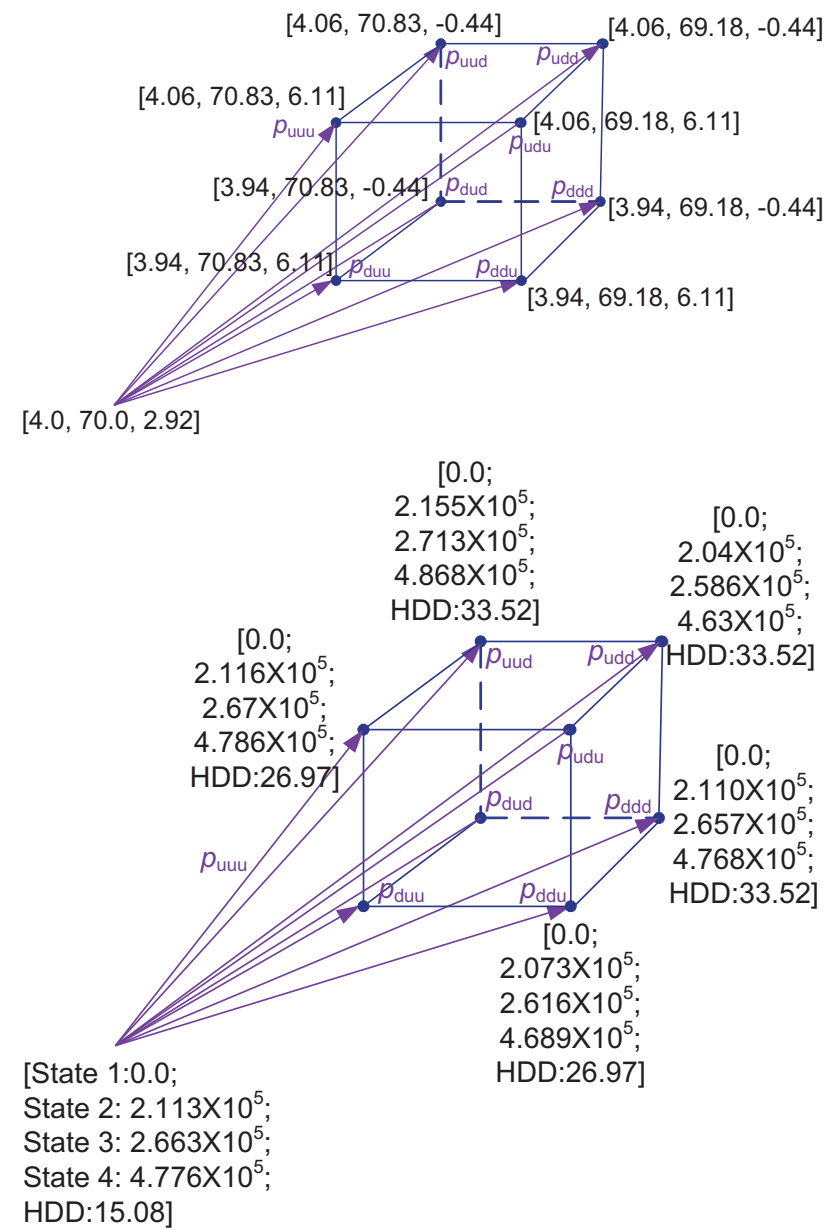

Figure 5.5: (Top) The lattice showing movements of natural gas price, electricity price and temperature. (Bottom) The corresponding values of spark spread at different states of the plant and accumulated HDD.

positive values in Figure 5.7 indicate profits from exercising weather options as a result of lower average HDD values (less than the strike heating degree days, $K$ ). The negative values indicate the expenses of paying the premium, since accumulated average path dependent HDD values in the corresponding nodes of the lattice plane are higher than the strike heating degree days, $K$.

The standard deviation of the spark spread option payoff corresponding to Figure 5.6 is $\sigma_{\pi}=$ 25918.07 and the standard deviation of weather option payoff corresponding to Figure $5.7 \sigma_{\omega}=$ 829.04. The correlation coefficient, $\gamma$, is -0.5299 . Therefore, the number of options, $n$, to buy at $t=1$ is determined from Equation $(5.16) n_{s=2}=16.57$. In a similar fashion the number of weather contracts obtained are: $n_{s=1}=0, n_{s=3}=18.41$, and $n_{s=4}=34.97$. Note that MATLAB (version 8.3) functions ' $\operatorname{std} 2()$ ' and 'corr2()' are used to calculate the standard deviation of of a matrix and the correlation coefficient between two matrices, respectively. 


\begin{tabular}{|l|l|l|l|l|l|l|}
\hline 212440.44 & 200933.03 & 189692.74 & 178713.38 & 167988.88 & 157513.33 & 147280.96 \\
\hline 219781.89 & 208274.48 & 197034.19 & 186054.83 & 175330.33 & 164854.78 & 154622.40 \\
\hline 226907.99 & 215400.58 & 204160.30 & 193180.93 & 182456.43 & 171980.89 & 161748.51 \\
\hline 233825.08 & 222317.66 & 211077.38 & 200098.01 & 189373.52 & 178897.97 & 168665.59 \\
\hline 240539.26 & 229031.85 & 217791.57 & 206812.20 & 196087.70 & 185612.16 & 175379.78 \\
\hline 247056.51 & 235549.10 & 224308.81 & 213329.45 & 202604.95 & 192129.40 & 181897.03 \\
\hline 253382.59 & 241875.18 & 230634.90 & 219655.53 & 208931.03 & 198455.49 & 188223.11 \\
\hline
\end{tabular}

Figure 5.6: Spark spread option payoffs at State 2 of the power plant at $t=7$.

\begin{tabular}{|c|c|c|c|c|c|c|}
\hline 1986.64 & 1986.64 & 1986.64 & 1986.64 & 1986.64 & 1986.64 & 1986.64 \\
\hline 1707.41 & 1707.41 & 1707.41 & 1707.41 & 1707.41 & 1707.41 & 1707.41 \\
\hline 1258.72 & 1258.72 & 1258.72 & 1258.72 & 1258.72 & 1258.72 & 1258.72 \\
\hline 800.01 & 800.01 & 800.01 & 800.01 & 800.01 & 800.01 & 800.01 \\
\hline 341.30 & 341.30 & 341.30 & 341.30 & 341.30 & 341.30 & 341.30 \\
\hline-117.42 & -117.42 & -117.42 & -117.42 & -117.42 & -117.42 & -117.42 \\
\hline-304.41 & -304.41 & -304.41 & -304.41 & -304.41 & -304.41 & -304.41 \\
\hline
\end{tabular}

Figure 5.7: Weather options payoff at $t=7$.

\subsubsection{Weather option payoff}

The payoff from exercising weather options is calculated based on Equation (5.10). As discussed in Section 5.2.2, for a weekly exercised weather options, the HDD is accumulated over seven days. The tick size, $\tau$, is $\$ 20$ per HDD. The strike HDD, $K$, is taken as 10 yrs historical weekly mean of the actual temperature data. The option premium to ba paid at $t=1$ is calculated from Equation (5.12) and is found to be 303.98 .

\subsection{Numerical results}

The power plant characteristics including turbine generation capacities, input-output coefficients, operations hour per day, plant operations and maintenance costs, startup and shutdown costs are assumed to be similar as in Chapter 4 described in Section 4.4.1 and Section 4.8. For the sake of simplicity, $Q_{g}^{d}$ is assumed to be $18^{0} \mathrm{C}$ and $\Delta Q_{g}^{d}$ is assumed to be $15.56^{0} \mathrm{C}$ (Tseng et al. 2009). The market price of risk, $\lambda$, for temperature in this Chapter is assumed to be a constant with a value equal to zero. However, a sensitivity test with respect to the market price of risk for temperature is also presented in Section 5.5.4, if the market price of risk varies from 0 to 0.40 . 


\subsubsection{The expected value of the power plant}

The expected value of the power plant at different states of the plant under different winter conditions is shown in Figure 5.8. The observations deduced from this result are: (i) When the monthly expected HDD is high, representing a cooler winter in the month of January, the power plant's expected value is contributed by the spark spread option only. (ii) When the month of January is not-so-cold with a lower HDD, the expected value from the spark spread option tends to moving downward. The weather put option is then began to be exercised as the winter becomes warmer. The power plant's expected value is then contributed from exercising the weather put option. (iii) The consequence of using weather option is more identifiable at State 4, when both turbines are operating. While the expected spark spread option value drops sharply at State 4 at lower expected HDD values, exercises of weather put option add value to this downward the spark spread option value and raise the overall expected value of the power plant. (iv) The historical monthly average HDD for the month of January in the city of Toronto, Canada over the period of 2005-2015 is around 720, while the maximum monthly HDD is 830 and the minimum monthly HDD is 552 . Figure 5.8 shows that when the expected HDD value falls below 650 at State 4, the weather option gets exercised and contributes to the power plant's expected value. However, at states 2 and 3, the weather put option gets exercised at around the HDD value of 630, representing a more warmer winter. (v) The 'solid' line in the figure represents the expected value with switching flexibility of the power plant. It is observed that the flexibility adds values to the power plant. The expected value of the power plant with switching flexibility is higher than the expected value of the plant without switching flexibility. Because, the power plant tends to switch from a lower plant value to a higher plant value and thus adds more value to the power plant. When both turbines of the plant are in operation at State 4, the expected values with and without switching flexibility merges, as there is no room for switching to a state with a higher expected value. (vi) It is observed that for a more warmer winter condition, around the expected HDD of 620, the payoff from exercising the weather option increases sharply. However, weather derivative contracts can be capped in order to create a liquid market active with potential buyers and sellers (Geman 2000).

Similar to observations for the month of January is found in Figure 5.9 for the month of February. The weather option begins to play its role in the course of warmer winter, when the expected monthly HDD falls below 410 at State 4, and 390 at states 2 and 3. Note that the historical monthly mean HDD for the month of February over the period of 2005-2015 is around 600. The results obtained from the proposed model reflects that while the expected HDD is higher than the historical mean HDD indicating a cooler February, the weather put option remains unexercised. On the other hand, when the expected HDD is lower than the historical mean HDD representing 

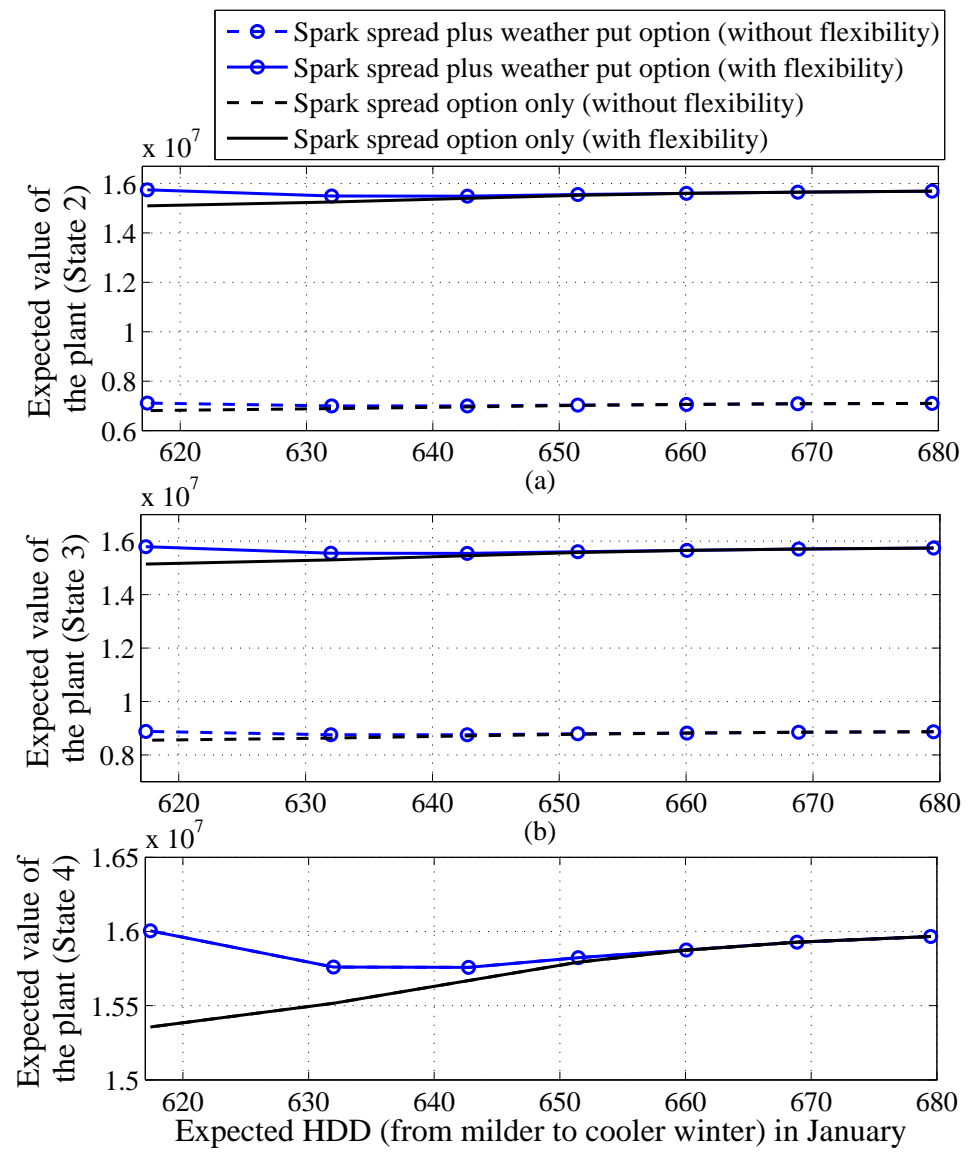

(c)

Figure 5.8: Expected value of the power plant with and without weather options at different expected HDD in the month of January: (a) State 2, (b) State 3, and (c) State 4.

a milder winter, the weather put options get exercised and contributes to the expected value of the power plant. In a more warmer winter, the payoff from wether option becomes active and contributes more to the expected value of the power plant.

Figure 5.10 shows the expected value of the power plant over a season of two-month period of January and February. It is observed that as the HDD increases with a cooler winter season, the expected value based on spark spread option increases. There is, on the other hand, an increasing trend in the expected value from the integrated spark spread and weather options as the season becomes warmer.

Figure 5.11 shows how the different values of $R^{c}$ affect the expected value of the power plant. The expected values of the power plant both with and without weather put options increase as the value of $R^{c}$ increases. The reason is that a higher value of $R^{c}$ induces more power generation in a cold environment temperature. The discussion in Section 5.2.1 also corroborates this fact. It is also observed in Figure 5.11 that the expected spark spread option value starts to diminish at a lower HHD in a warmer winter, while the expected payoff from the weather option begins to be 

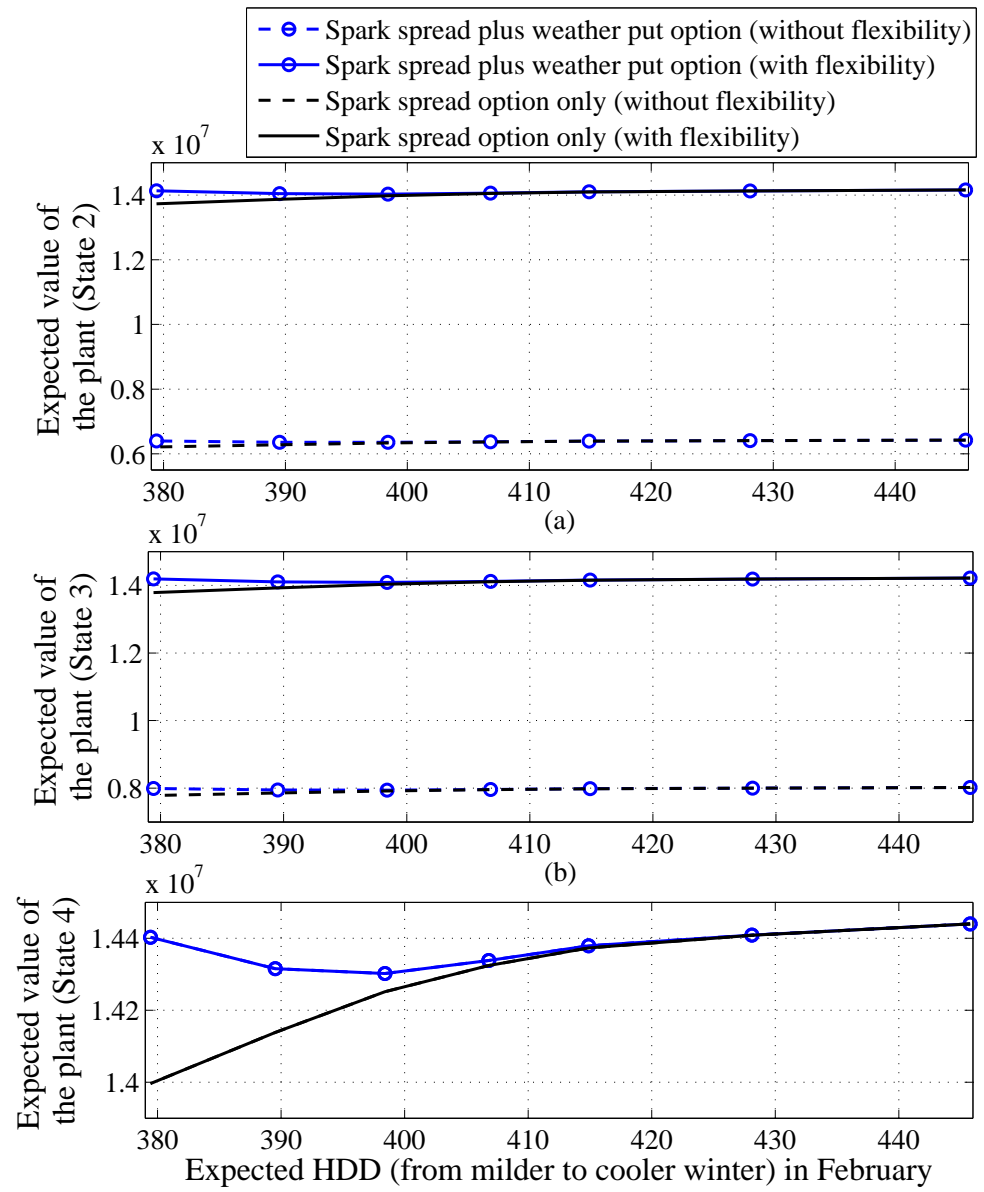

(c)

Figure 5.9: The expected values of the power plant with and without weather options at different expected HDD in the month of February: (a) State 2, (b) State 3, and (c) State 4.

activated.

\subsubsection{Sensitivity of the expected value to changes in the volatilities}

To examine the effect of volatilities, the volatility parameters are varied $\pm 10 \%$ from the value stated in Section 5.3.1. For the month of February, Figure 5.12 shows the expected value of the power plant with and without using weather derivatives as the electricity volatility increases. It is observed that the expected value of the power plant with and without using weather derivatives increases as the electricity volatility increases.

Figure 5.13 shows the effect of varying the the natural gas price volatility on the power plant's expected value. It is observed that the expected value from spark spread option tends to decreases as the natural gas price volatility increases. A higher uncertainty in the natural gas price represents a higher uncertainty in the cost component of the expected value, that in turn, results in a reduced expected value. The decreasing trend in the spark spread option value triggers the weather option 

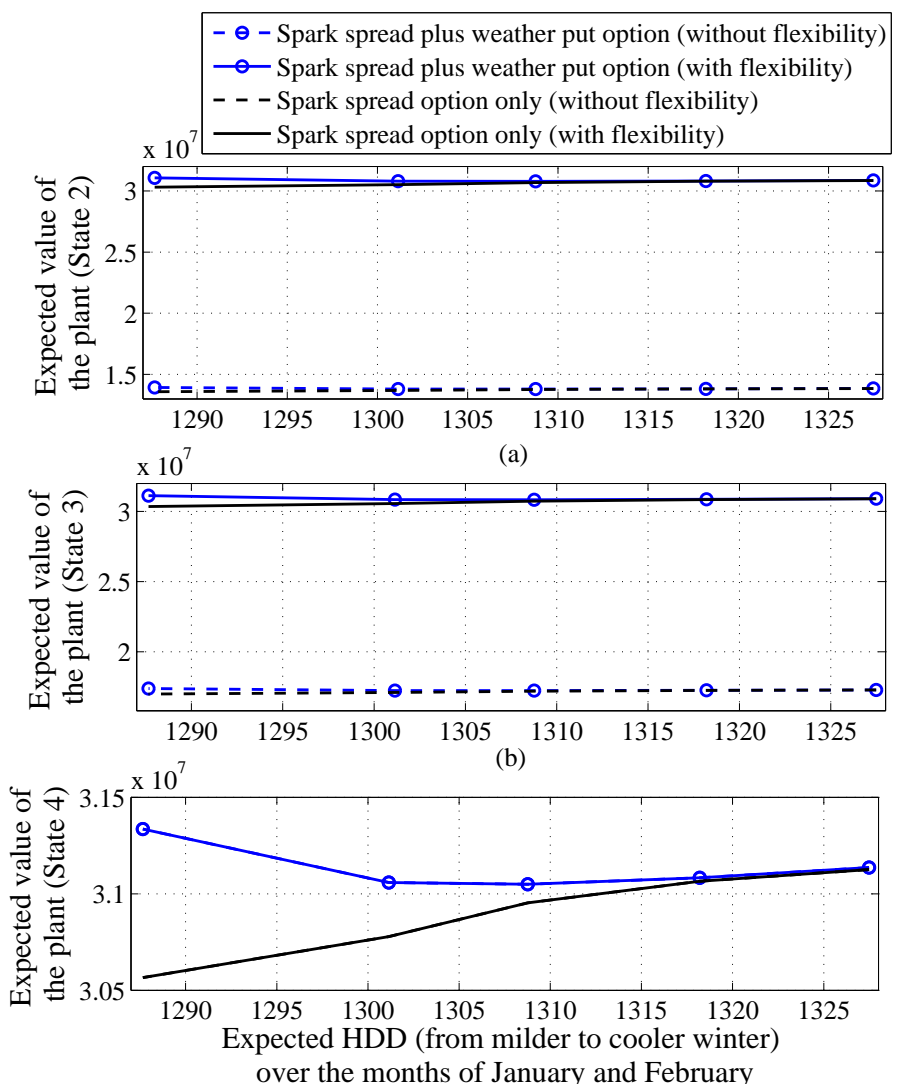

(c)

Figure 5.10: The expected values of the power plant with and without weather options at different expected HDDs over the winter season combining the months of January and February: (a) State 2, (b) State 3, and (c) State 4.

payoff to be exercised and hence the expected power plant value using weather option along with spark spread option tends to increase as the volatility of natural gas price increases.

The effect of temperature volatility on the power plant's expected value is shown in Figure 5.14. As the uncertainty in temperature increases, the expected values of the power plant tends to increase. It is also observed that weather option adds higher values at the higher temperature volatility.

\subsubsection{Sensitivity of the expected value to changes in the correlation coefficient}

Figure 5.15 shows the effect of the correlation coefficient between electricity prices and temperature on the expected value of the power plant for the month of February. It is observed that the expected value from the spark spread option tends to decrease as the the correlation coefficient moves from -1 to +1 . The negative correlation coefficient refers that the electricity price and temperature moves in opposite direction. As the electricity price increases, the expected value tends to increase and at the same time due to negative correlation, the decreasing temperature also tends to increase the 


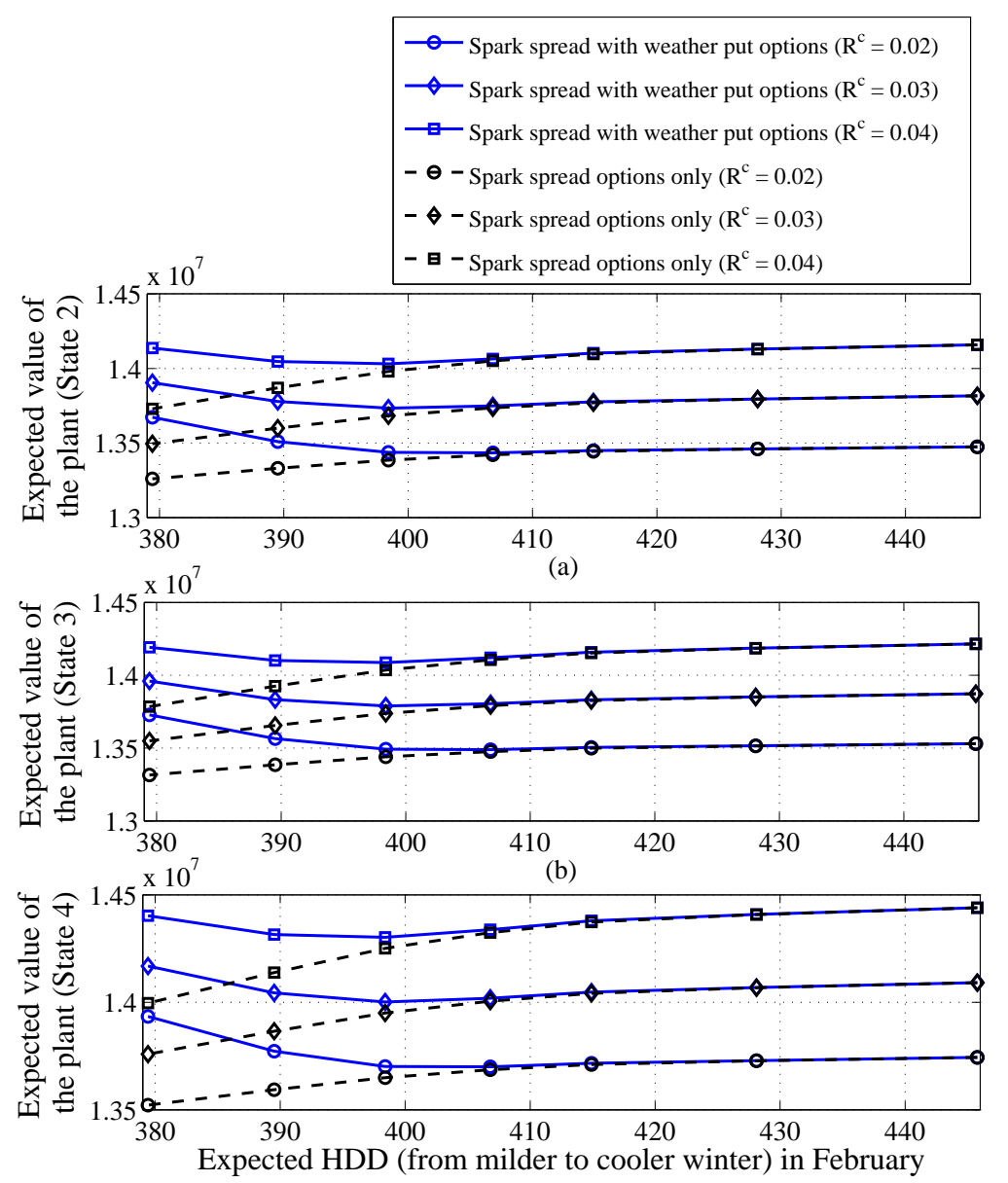

(c)

Figure 5.11: Expected value of the power plant with and without weather options at different values of $R^{c}$ : (a) State 2, (b) State 3, and (c) State 4.

expected spark spread option value. For this reason, when the correlation coefficient is negative, the expected value from the spark spread option is higher than that of when the correlation coefficient is positive. When the coefficient of correlation is positive, the weather option tends to add values to the downward spark spread option values. The reverse phenomenon is observed in Figure 5.16 with respect to changes in the correlation coefficient between the natural gas prices and temperature. Weather option tends to add values when the correlation coefficient between the natural gas prices and temperature is negative.

\subsubsection{Sensitivity of the expected value to market price of risk for temperature}

As discussed in Section 3.1, for a non traded variable like temperature deducting the risk premium from the process drift would provide a risk-neutral valuation world. The risk premium is dependent on the market price of risk. Cao and Wei (2004) study the market price risk for temperature based on the Lucas equilibrium asset-pricing model. They conclude that the market price of risk for 

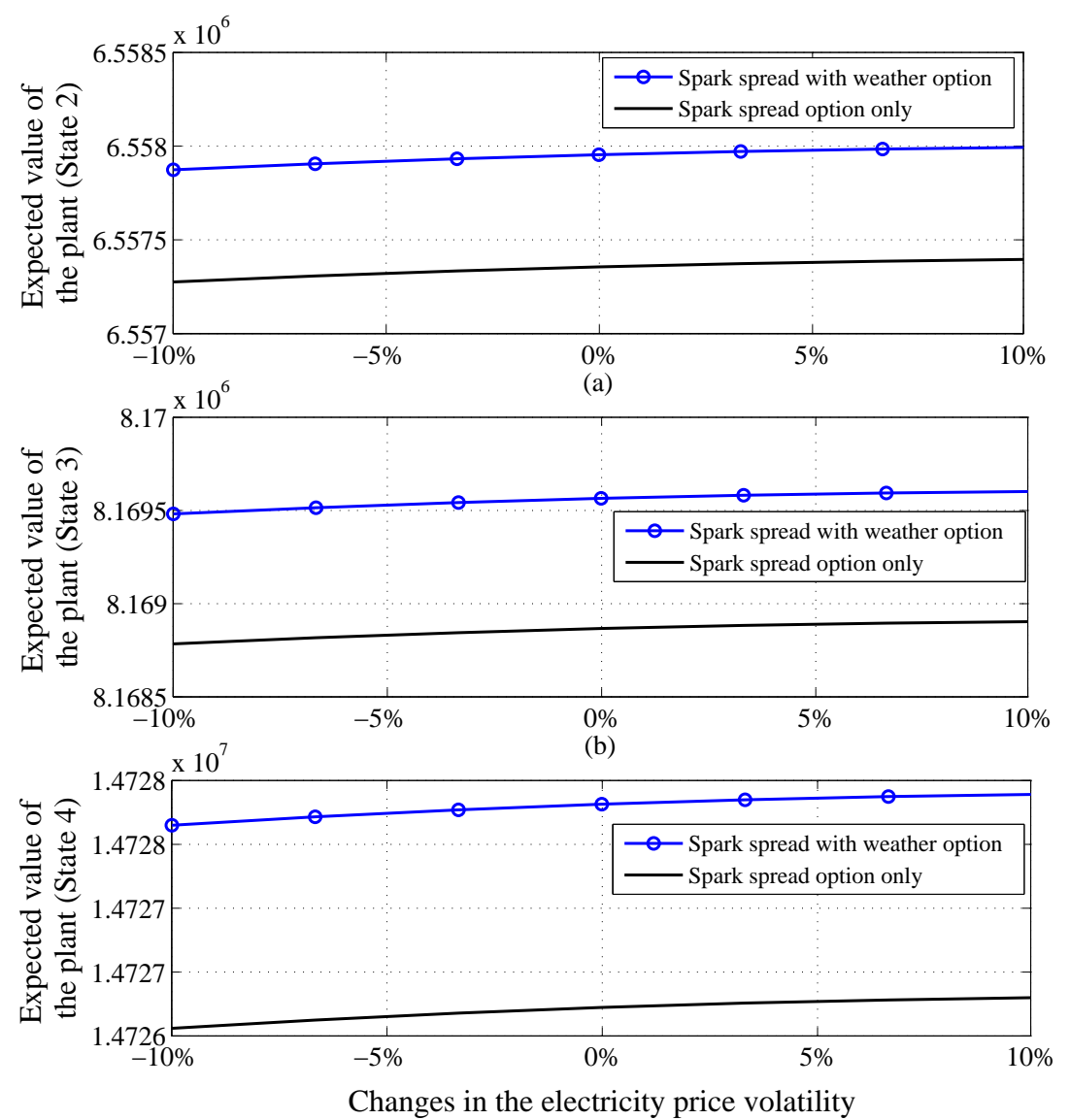

(c)

Figure 5.12: Sensitivity of the expected value of the power plant to the changes in electricity price volatility: (a) State 2, (b) State 3, and (c) State 4.

temperature can be approximated to be zero when the correlations between the temperature process and the consumption are very low and/or the risk aversion is low. Figure 5.17 shows the sensitivity of the expected value of the plant with respect to market price of risk for temperature as it changes from 0 to 0.40 . It is observed that as the market price of risk increases, the expected values of the plant also increases. Since an increasing market price of risk reduces the temperature drift, the heating degree days then tend to increase that results in an increase in the expected spark spread option value of the plant. However, the increasing trend of the expected value from exercising the weather option tends to slow down as the market price of risk for temperature increases. 


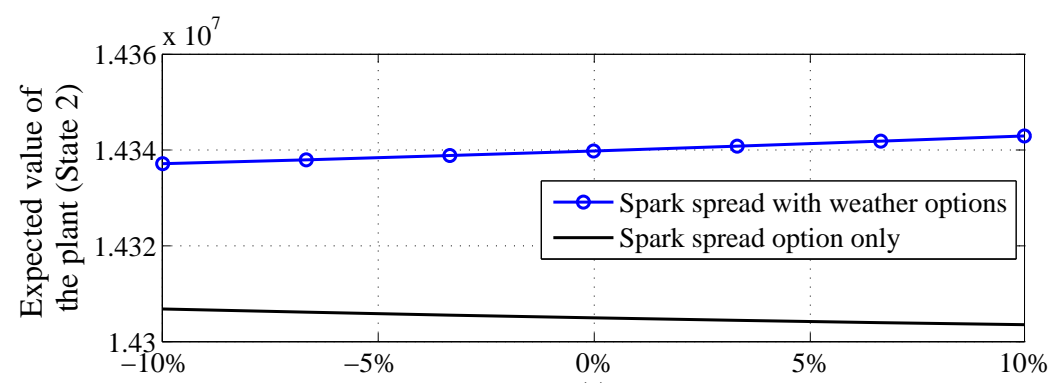

(a)
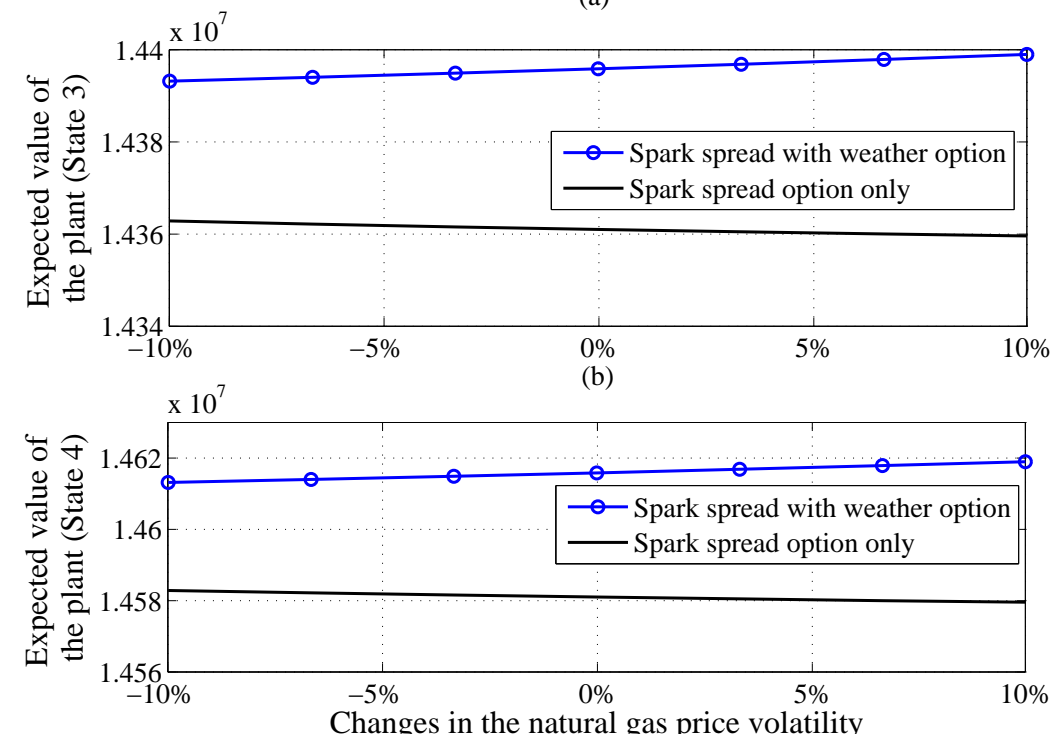

(c)

Figure 5.13: Sensitivity of the expected value of the power plant to the changes in natural gas price volatility: (a) State 2, (b) State 3, and (c) State 4. 

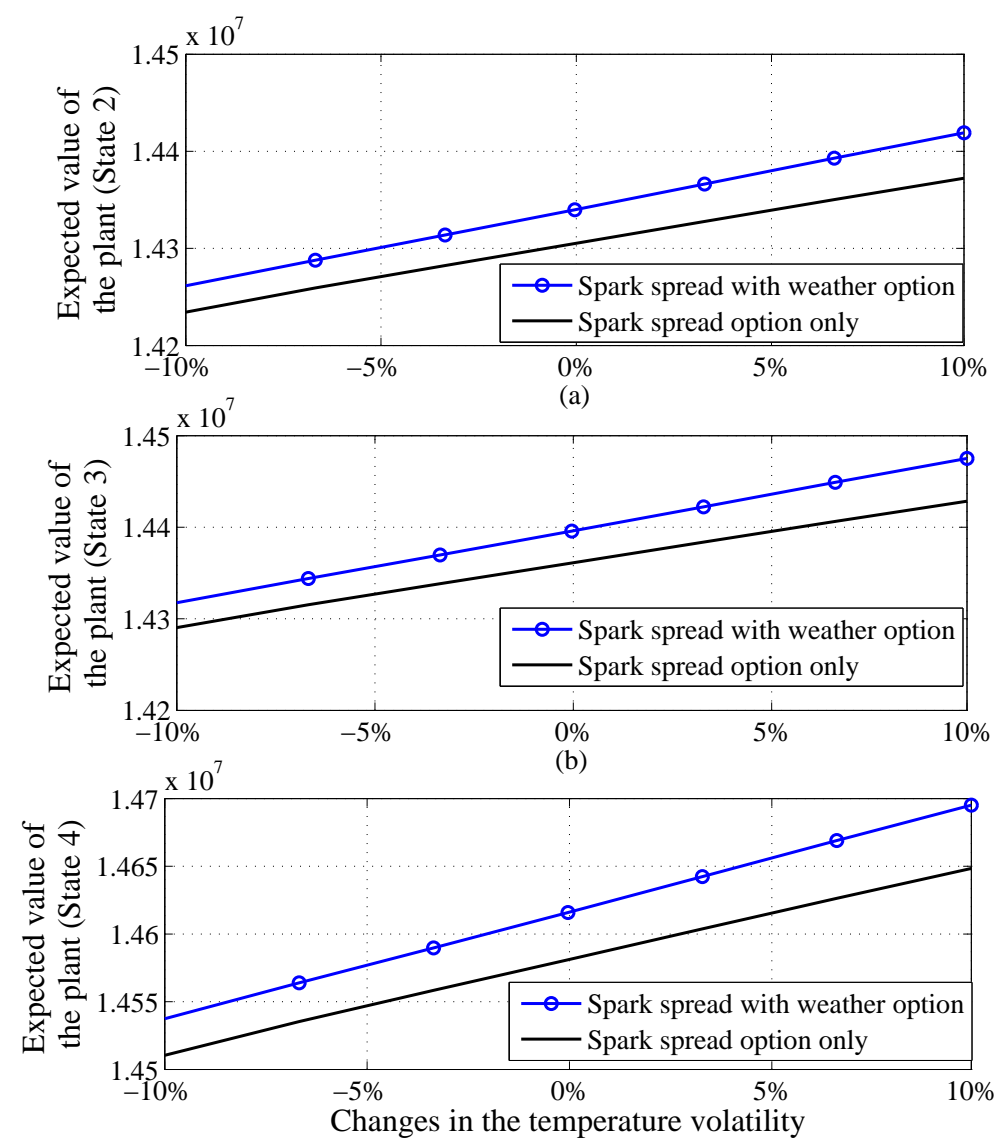

(c)

Figure 5.14: Sensitivity of the expected value of the power plant to the changes in temperature volatility: (a) State 2, (b) State 3, and (c) State 4. 

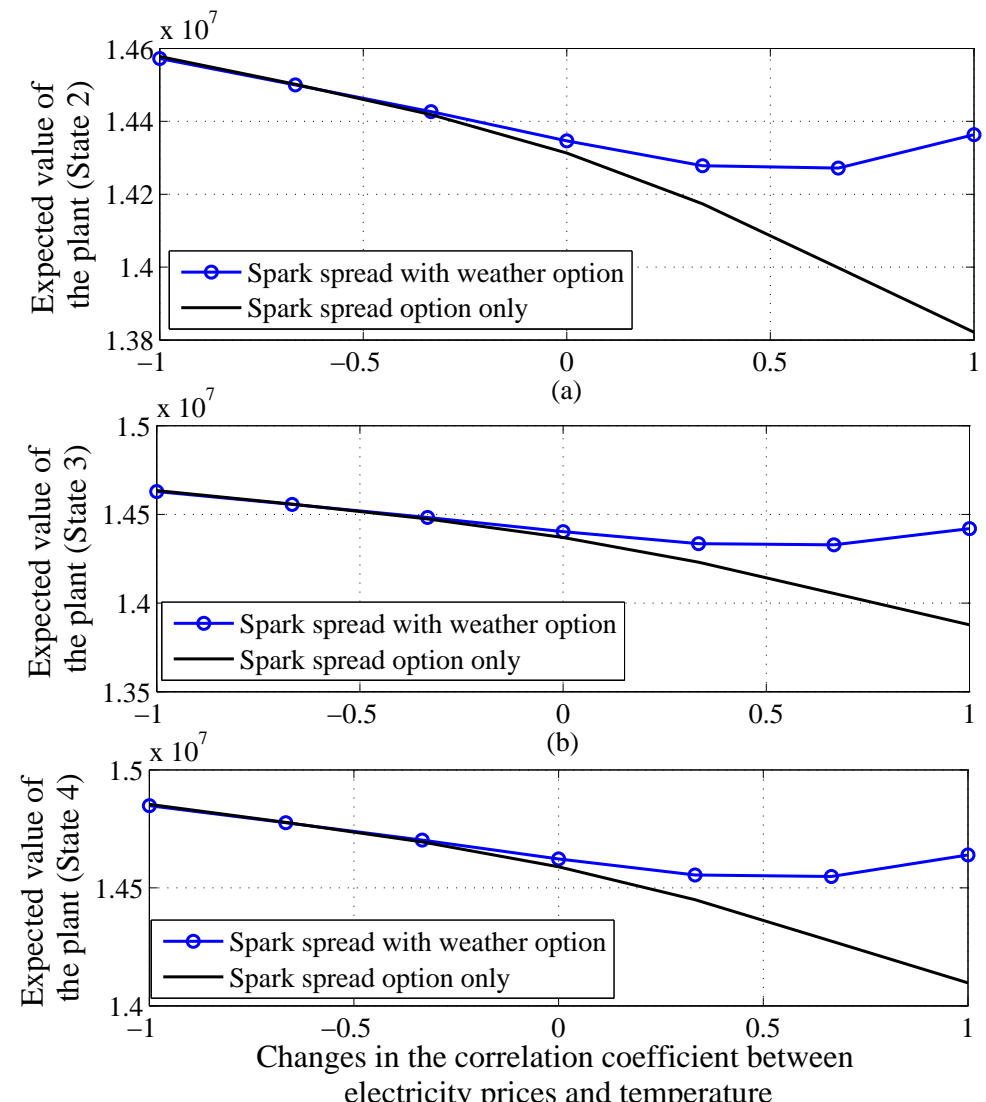

(c)

Figure 5.15: The expected value of the power plant with respect to the correlation coefficient between electricity price and temperature: (a) State 2, (b) State 3, and (c) State 4. 

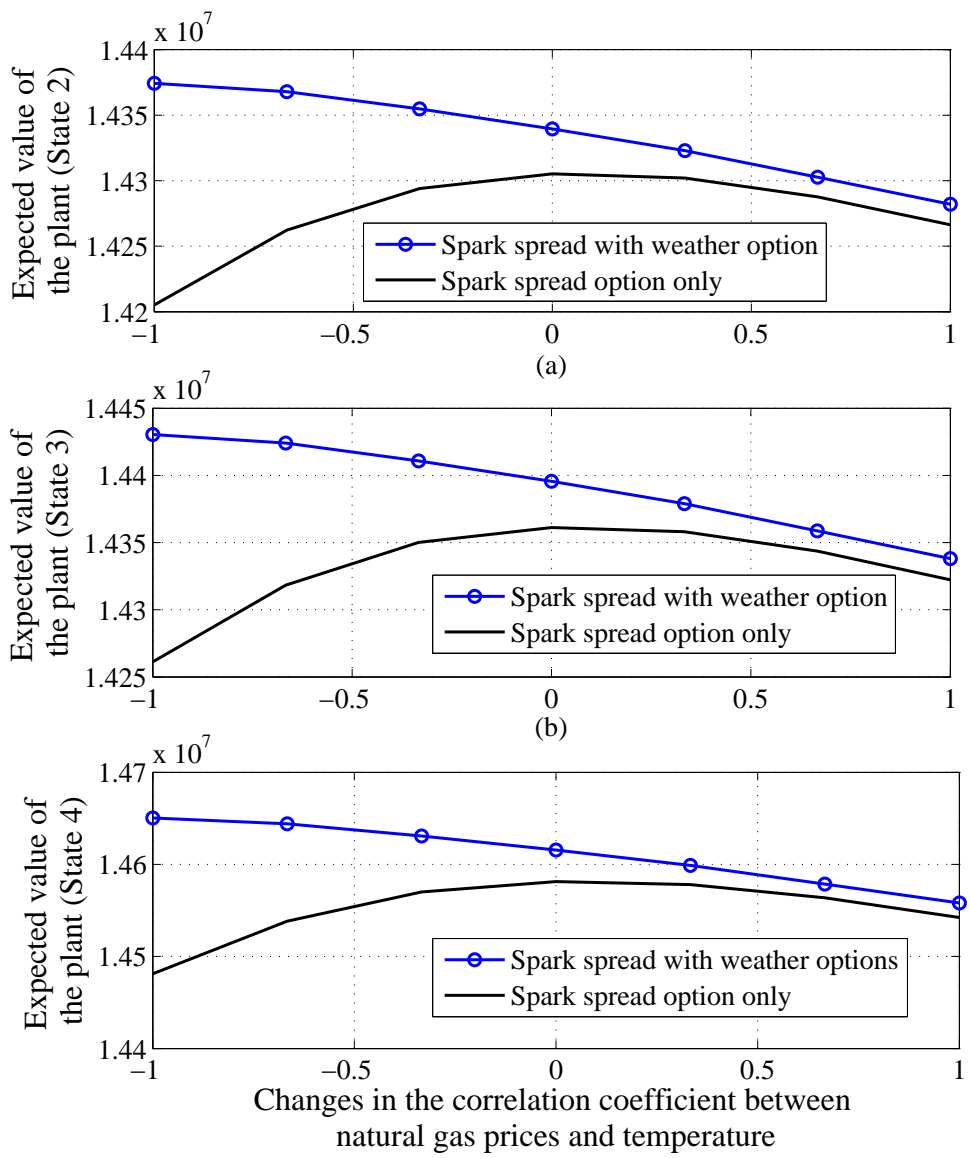

(c)

Figure 5.16: The expected value of the power plant with respect to the correlation coefficient between natural gas price and temperature: (a) State 2, (b) State 3, and (c) State 4.

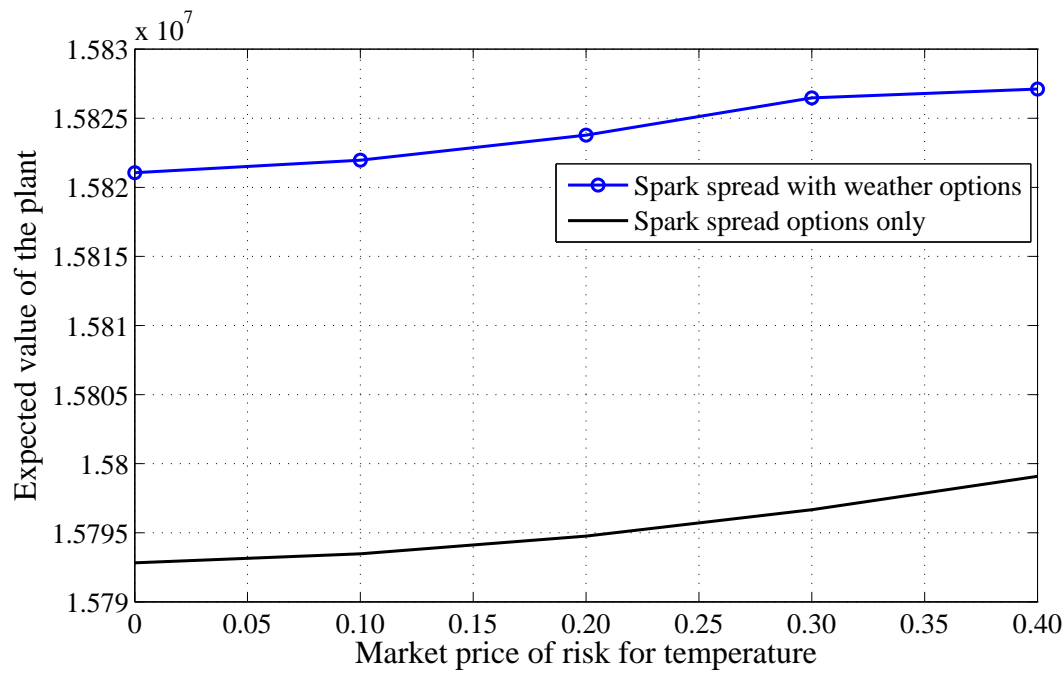

Figure 5.17: Expected value of the power plant with respect to the market price of risk for temperature. 


\subsection{Summary}

This chapter investigates the integration of spark spread and weather options for valuing a natural gas-fired power plant with multiple turbines. The motivation is to examine the implication of incorporating the environment temperature into the spark spread option based valuation model. The model utilizes the fact that a gas turbine usually performs better in a cooler environment. Moreover, a cooler winter prompting more heating requirements could generate a higher payoff from exercising the spark spread option. On the other hand, a not-so-cold winter, as referred to a warmer winter, causing a lower heating requirements is expected to generate a lower spark spread option value of the power plant. In an anticipation of a warm winter, the power plant owner could opt a long position in a temperature-based weather put option, which can be exercised when the accumulated heating degree days drops below a threshold or strike heating degree day. Against this backdrop, a lattice based numerical approach has been presented that depicts the correlated movement of electricity price, natural gas price and temperature. All of these three variables are assumed to follow mean reverting processes.

The power plant is assumed to possess multiple turbines. The turn-on and turn-off capabilities of these turbines according to the price movements and temperature behavior allow the power plant to be at different states and provide an opportunity of flexibility of switching among the states. The model determines the number of weather contracts to buy at each state of the plant by minimizing the variance of the total payoff. The expected values of the power plant with and without utilizing switching flexibility along with and without weather options are presented.

Results demonstrate that the expected value of the plant based on the spark spread option is higher in a cooler winter than that of in a warmer winter. However, the downward trend in the expected value in a warmer winter could be compensated by exercising the weather put option, when the expected HDD falls below the contract or strike heating degree day. It is found that as the expected HDD drops, denoting a more warmer winter, the expected value of the plant tends to increase by the exercises of the weather option. It is also observed that the flexibility of switching among the different states of the power plant adds values to the plant.

Sensitivity tests with respect to the changes in volatilities and the correlation coefficients are also presented. With the numerical setup used in this thesis, it is observed that the expected value of the power plant increases with an increase in the electricity price volatility. An increase in the volatility of the natural gas price, however, tends to decrease the expected spark spread option value, while the expected value from weather option tends to increase. It is observed that an increase in temperature volatility increases the expected values of the power plant. With regard to the changes in the correlation coefficients, it is observed that weather option adds values to the 
power plant when the correlation coefficient between the electricity prices and temperature changes from 0 to +1 . On the other hand, when the correlation coefficient between the natural gas prices and temperature is negative, weather option adds values to the power plant. The next chapter presents the valuation model for pricing weather option. 


\section{Chapter 6}

\section{The Valuation of Temperature-based Weather Options}

\subsection{Introduction}

This chapter presents the valuation model for pricing weather derivatives. Several studies in the literature outline the pricing problems of weather derivatives. The Ornstein-Uhlenbeck meanreverting process in different forms dominates the literature on modeling the stochastic behavior of temperature. Examples include Dornier and Queruel (2000), Alaton et al. (2002), Brody et al. (2002), Benth and Šaltytė-Benth (2005), and Benth et al. (2007). However, a single stochastic process may not be able to describe the dynamics of temperature appropriately and a switching behavior for temperature from one stochastic process to another may exist. This type of modeling approach leads to regime-switching models for temperature, in which temperature undergoes discrete shifts in mean and volatility as latent states. A variety of regime-switching models can be developed based on different stochastic processes for temperature in different regimes. Accordingly, the one of the objectives of this thesis is to examine different regime-switching models capturing the temperature dynamics and compare their performance with a single-regime model. Three Markov regime-switching models are proposed and their performances are compared with that of a single-regime Ornstein-Uhlenbeck mean-reverting model.

Model 1 assumes that daily temperature switches between two regimes: one regime is governed by a mean-reverting process and the other by a Brownian motion. Model 2 assumes that daily temperature switches between two regimes and each regime is governed by a Brownian motion. Model 3 assumes that each regime is governed by a mean-reverting process in which the mean and speed of the mean-reversion remain the same, but only the volatility switches between the states. Each Markov regime-switching model is represented by a pentanomial lattice. Model 
4 assumes that daily temperature follows a mean-reverting process, which is represented by a binomial lattice proposed by Nelson and Ramaswamy (1990). For each of these four models, the average expected monthly and seasonal HDD (CDD) are calculated and then they are compared with the actual average monthly and seasonal HDD (CDD), respectively. This part of the research has been published in Elias et al. (2014).

\subsection{Two-state regime-switching models for Toronto temperature data}

The regime-switching behavior in electricity prices is prompted by spikes that are often caused by short-term instability between supply and demand (Huisman and Mahieu 2003). Similarly, abrupt changes in temperature - caused by a combination of several factors including latitude, intensity of solar circulation, land and water surface areas, ocean currents, elevation, and clear skies (Ahrens 2006) - induce the regime-switching behavior in temperature. The presence of abrupt changes in the annual and seasonal temperature time series in the Greater Toronto Area (GTA) is detected by Mohsin and Gough (2010). A report from Environment Canada (Francis and Hengeveld 1998) states that "climates of the past, rather than changing gradually, have sometimes shown a tendency, at least regionally, to switch abruptly to a radically different mode." Studies find that temperature is the main impetus for switching the climate's state abruptly to a different radical mode. The Environment Canada report also states that a shift of $0.5^{\circ} \mathrm{C}$ increase in the mean temperature would increase the number of hot days above $35^{\circ} \mathrm{C}$ by $25 \%$ in Victoria, Australia. In the city of Toronto, an increase of $4^{\circ} \mathrm{C}$ in the mean temperature would increase the number of hot days above $30.5^{\circ} \mathrm{C}$ from 1 in 10 days to 1 in 2 days, i.e., from $10 \%$ to $50 \%$, in a summer (Francis and Hengeveld 1998). Huisman (2008) also shows that a shift in mean temperature induces regimeswitching behavior in electricity prices and increases the probability of spike occurrences. These factors provide clues to examine the regime-switching behavior of Toronto temperatures.

The average daily temperature of Toronto, Canada, as measured at Pearson International Airport from January 1, 2005 to December 31, 2008, for a total of 1461 days was collected from the website of National Climate Data and Information Archive of Canada. ${ }^{1}$ The temperature ranges from a minimum of $-20.3^{\circ} \mathrm{C}$ to a maximum of $31.5^{\circ} \mathrm{C}$. The average temperature on day $t, Q_{t}$, can

\footnotetext{
${ }^{1}$ When dealing with weather data, data cleaning is important before any pricing method is applied. There are two types of temperature data: 'synoptic' and 'climate'. 'Synoptic' data are used for immediate feeding into weather forecast without data cleaning and quality checking. 'Climate' data are purely preserved for historical purpose and therefore pass through several levels of data cleaning and quality control in order to remove absurd values and fill gaps. Because of higher level of accuracy and reliability, 'climate' data are used for weather contracts (Jewson and Brix 2005). The data used in this study are obtained from Environment Canada's database of historical 'climate' data and can be used reliably for weather contracts.
} 
be modeled as the sum of the deseasonalized stochastic component, $w_{t}$, and deterministic annual seasonality component, $S_{t}$, as follows:

$$
Q_{t}=w_{t}+S_{t}
$$

$S_{t}$ can be further approximated as:

$$
S_{t}=A_{1} \sin \left[2 \pi\left(t+A_{2}\right) / 365\right]+A_{3} t+A_{4},
$$

where $A_{1}, A_{2}, A_{3}$ and $A_{4}$ are constants (e.g., Weron 2007). The values of these constants shown in Table 6.1 are estimated by a Gauss-Newton non-linear least square regression on time $t$, where $t=$ $1,2, \ldots, 1461$, using a RATS (Regression Analysis of Time Series) function named "NLLS" (Enders 2003). Figure 6.1 (top) shows the average daily temperature (average over daily minimum and maximum) along with the fitted seasonality component, $S_{t}$. After removing $S_{t}$, the deseasonalized stochastic component, $w_{t}$, can be expressed as: $w_{t}=Q_{t}-S_{t}$.
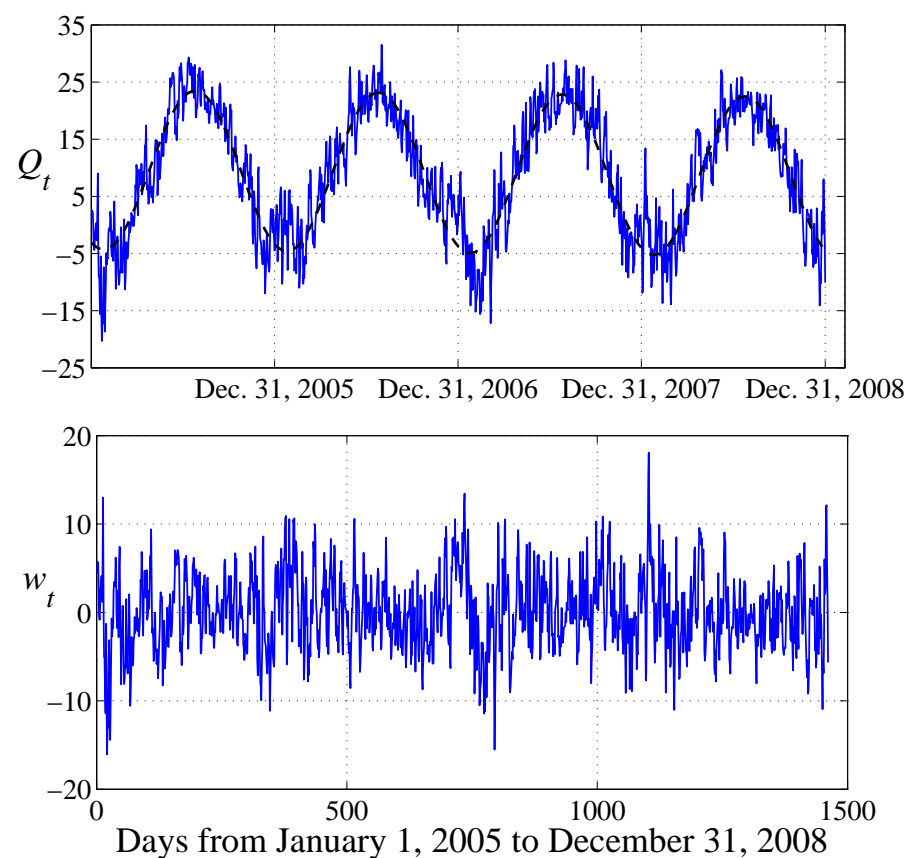

Figure 6.1: (Top) Average daily temperature (in Celcius), $Q_{t}$, with seasonality, $S_{t}$. (Bottom) Deseasonalized stochastic component, $w_{t}$.

In this study, the temperature dataset of Toronto, Canada, is examined for the regime switching behavior. The regime-switching parameters are estimated using the log-likelihood function maximized by the Broyden-Fletcher-Goldfarb-Shanno (BFGS) method in RATS (Brooks 2009). Figure 6.2 (top) shows the presence of two regimes (or states) in solid and dashed lines and Figure 6.2 (bottom) depicts the smoothed probability of being in the corresponding states. To clearly show 
Table 6.1: Coefficients of $S_{t}$, the deterministic component of Toronto average daily temperature

\begin{tabular}{c|c|c|c|c}
\hline Variable & Coefficient & Standard error & T-statistic & $\mathrm{p}$-value \\
\hline$A_{1}$ & 13.94 & 0.16 & 86.05 & 0.00 \\
$A_{2}$ & -115.62 & 0.68 & -168.91 & 0.00 \\
$A_{3}$ & -0.001 & 0.00 & -3.21 & 0.00 \\
$A_{4}$ & 9.69 & 0.23 & 41.78 & 0.00 \\
\hline
\end{tabular}

the two states (regimes), a snapshot of the first five hundred days of the dataset is taken. The regime parameters based on daily temperature data are reported in Table 6.2 in Section 6.4.1.
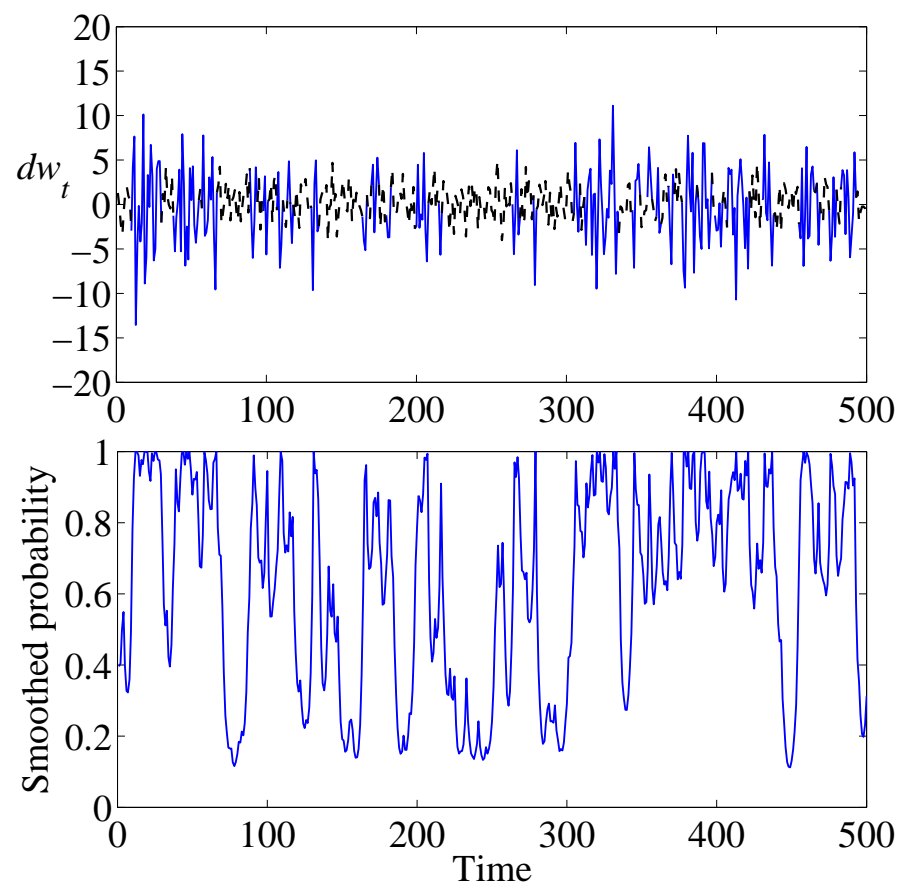

Figure 6.2: The smoothed probability of being in two states.

\subsection{The Models}

This section develops three regime-switching models and one single-regime model and their underlying processes along with construction of corresponding lattices for each model.

\subsubsection{Model 1: With a mean-reverting process and a Brownian motion}

A two-state regime-switching model for the stochastic component of the temperature, $w_{t}$, can be represented by $w_{t, i}$, if $w_{t}$ is in regime $i$, with unconditional probability $p_{i}$, for $i=1,2$, where 
$\sum_{i=1}^{2} p_{i}=1$. Accordingly,

$w_{t}=\left\{\begin{array}{l}w_{t, 1} \text { if } w_{t} \text { is in regime } 1 \text { with probability } p_{1}, \text { where } d w_{t, 1}=\kappa_{w}\left(\bar{w}-w_{t, 1}\right) d t+\sigma_{1} d B_{w}, \\ w_{t, 2} \text { if } w_{t} \text { is in regime } 2 \text { with probability } p_{2}, \text { where } d w_{t, 2}=\mu_{2} d t+\sigma_{2} d B_{w},\end{array}\right.$

where $\kappa_{w}$ is the speed and $\bar{w}$ is the mean of the mean-reverting process, $\mu_{2}$ is the mean of the Brownian motion, $\sigma_{1}$ and $\sigma_{2}$ are the volatilities of the mean-reverting and Brownian motion processes, respectively, and $d B_{w}$ is the increment of the Wiener process. Since temperature is non-tradable, it is necessary to determine the risk-neutral equivalent drift that reflects the risk of the option. The risk-neutral equivalent drift is obtained by subtracting the risk premium from the actual growth rate. The amount of the risk premium depends on the market price of risk. Therefore, if the market price of risk is known, the risk-neutral equivalent drift can be computed (Wahab et al. 2010). Cao and Wei (2004) assess the significance of the market price of risk in the valuation of weather futures and options based on the extended Lucas equilibrium asset-pricing model, in which uncertainties in economy are simulated by aggregate dividend (i.e., total consumption) and a state variable represented by temperature. They find that the market price of risk is significant, if the correlation between the aggregate dividend and temperature is high and the risk aversion parameter is also high. Their empirical study finds the largest risk premium of $11.61 \%$ for call options for the city of Dallas, USA. It may also be noted, referring to Hull (2002), that 'the market price of risk is not dependent on the nature of the derivative and at any time the market price of risk must be the same for all derivatives that are dependent on the same underlying variable.' Accordingly, considering the same market price of risk, $\lambda$, for both regimes, in Equation (6.3), $\bar{w}$ is replaced by $\left(\bar{w}-\lambda \sigma_{1} / \kappa_{w}\right)$ and $\mu_{2}$ is replaced by $\mu_{2}-\lambda \sigma_{2}$ (Weron 2007). In a two-state regime-switching model, there are four transition probabilities in the Markov chain for the evolution of the unobserved latent indicator variable and given by Equation (3.39). To construct the lattice, the step size of the regime modeled by the mean-reverting process is defined as (e.g., Nelson and Ramaswamy 1990):

$$
\hat{\Delta}_{1}=\sigma_{1} \sqrt{\Delta t}
$$

The step size of the other regime modeled by the Brownian motion can be defined as (e.g., Bollen 1998):

$$
\hat{\Delta}_{2}=\sqrt{\mu_{2}^{2}(\Delta t)^{2}+\sigma_{2}^{2} \Delta t}
$$

where $\Delta t$ represents the time interval between two layers of the lattice (see Appendix E). Note that $\Delta t$ can be chosen sufficiently small to make all conditional branch probabilities positive. 
In order to develop a pentanominal lattice for the two-state regime-switching model, each regime is represented by a trinomial lattice and the middle branch is shared by both regimes. To reduce the number of nodes in the lattice, the nodes are merged by adjusting the step size of one regime as follows, so that the step sizes of two regimes have a 1:2 ratio. Let the step sizes of regimes 1 and 2 , be given by $\hat{\Delta}_{1}$ in (6.4) and $\hat{\Delta}_{2}$ in (6.5), respectively. Define the indices $k_{1}:=\arg \min \left\{\hat{\Delta}_{i}: i=1,2\right\}$ and $k_{2}:=\arg \max \left\{\hat{\Delta}_{i}: i=1,2\right\}$, and set $\Delta_{i}=\hat{\Delta}_{k_{i}}$ for $i=1,2$. Thus, $\Delta_{1}<\Delta_{2}$ holds, and define $\Delta=\max _{i}\left\{\frac{\Delta_{i}}{i}\right\}$. Let $\Delta=\frac{\Delta_{i}}{i}$ (i.e., $\frac{\Delta_{i}}{i}>\frac{\Delta_{j}}{j}$ ), then the step size of $\Delta_{j}$ should be adjusted according to Equation (6.6).

$$
\Delta_{j}= \begin{cases}\Delta_{i}, & \text { if } j=i, \\ j \frac{\Delta_{i}}{i}, & \text { if } j \neq i .\end{cases}
$$

For each trinomial lattice, the three conditional branch probabilities for the upward, middle, and downward branches, respectively can be determined by matching the moments implied by the lattice to the moments implied by the distribution of the underlying process of the corresponding regime (Wahab et al. 2010).

For the mean-reverting process:

$$
\begin{aligned}
& \wp_{\Delta_{j}, u}= \begin{cases}\frac{1}{2}\left[\frac{\Delta_{j}{ }^{2}}{\left(j \frac{\Delta_{i}}{i}\right)^{2}}+\frac{\kappa_{w}\left(\bar{w}-w_{t, 1}\right) \Delta t}{j \frac{\Delta_{i}}{i}}\right] & \text { if } 0 \leq \frac{1}{2}\left[\frac{\Delta_{j}{ }^{2}}{\left(j \frac{\Delta_{i}}{i}\right)^{2}}+\frac{\kappa_{w}\left(\bar{w}-w_{t, 1}\right) h}{j \frac{\Delta_{i}}{i}}\right] \leq 1 \\
0, & \text { if } \frac{1}{2}\left[\frac{\Delta_{j}{ }^{2}}{\left(j \frac{\Delta_{i}}{i}\right)^{2}}+\frac{\kappa_{w}\left(\bar{w}-w_{t, 1}\right) \Delta t}{j \frac{\Delta_{i}}{i}}\right]<0 \\
1, & \text { otherwise. }\end{cases} \\
& \wp_{\Delta_{j}, d}= \begin{cases}\frac{1}{2}\left[\frac{\Delta_{j}{ }^{2}}{\left(j \frac{\Delta_{i}}{i}\right)^{2}}-\frac{\kappa_{w}\left(\bar{w}-w_{t, 1}\right) \Delta t}{j \frac{\Delta_{i}}{i}}\right] & \text { if } 0 \leq \frac{1}{2}\left[\frac{\Delta_{j}{ }^{2}}{\left(j \frac{\Delta_{i}}{i}\right)^{2}}-\frac{\kappa_{w}\left(\bar{w}-w_{t, 1}\right) \Delta t}{j \frac{\Delta_{i}}{i}}\right] \leq 1, \\
0, & \text { if } \frac{1}{2}\left[\frac{\Delta_{j}{ }^{2}}{\left(j \frac{\Delta_{i}}{i}\right)^{2}}-\frac{\kappa_{w}\left(\bar{w}-w_{t, 1}\right) \delta t}{j \frac{\Delta_{i}}{i}}\right]<0, \\
1, & \text { otherwise. }\end{cases} \\
& \wp_{\Delta_{j}, m}= \begin{cases}0, & \text { if } i=j, \\
1-\wp_{\Delta_{j}, u}-\wp_{\Delta_{j}, d}, & \text { otherwise. }\end{cases}
\end{aligned}
$$


For the Brownian motion:

$$
\begin{gathered}
\wp_{\Delta_{j}, u}=\frac{1}{2}\left[\frac{\Delta_{j}^{2}}{\left(j \frac{\Delta_{i}}{i}\right)^{2}}+\frac{\mu_{j} \Delta t}{\left(j \frac{\Delta_{i}}{i}\right)}\right], \\
\wp_{\Delta_{j}, d}=\frac{1}{2}\left[\frac{\Delta_{j}^{2}}{\left(j \frac{\Delta_{i}}{i}\right)^{2}}-\frac{\mu_{j} \Delta t}{\left(j \frac{\Delta_{i}}{i}\right)}\right], \\
\wp_{\Delta_{j}, m}=1-\wp_{\Delta_{j}, u}-\wp_{\Delta_{j}, d} .
\end{gathered}
$$

To determine the expected HDD or CDD using the lattice defined above, a three-step procedure is utilized: (1) develop a lattice for the stochastic component, $w_{t}$, of the temperature; (2) add the deterministic annual seasonal component, $S_{t}$, to the value of $w_{t}$ at each node of the lattice, to depict the behavior of the temperature, $Q_{t}$; and (3) calculate the expected HDD or CDD. For example, the lattice is initialized at the root node with the stochastic component of the temperature. The step sizes of the lattice are determined by using Equations (6.4) and (6.5). Necessary adjustments are made to merge the nodes efficiently as stated in Equation (6.6).

To calculate the expected HDD, the dynamic programming approach is used. For example, at a given node in period $t$,

$$
\operatorname{HDD}_{t, i}=\max \left(0, \bar{Q}-Q_{t}\right)+\sum_{j=1}^{2} \gamma_{i j}\left(\mathbf{E}\left[\operatorname{HDD}_{t+1, j}\right]\right) \text { for } i=1,2
$$

where $\mathrm{HDD}_{t, i}$ is the expected HDD at a given node in regime $i$ at period $t$. Expectations of future conditional HDD, $\mathbf{E}\left[\mathrm{HDD}_{t+1, i}\right]$, are taken using the appropriate conditional branch probabilities.

\subsubsection{Model 2: With two Brownian motions}

In this two-state regime-switching model, where each regime is governed by a Brownian motion, the stochastic component, $w_{t}$, of the temperature can be represented by $w_{t, i}$, if $z_{t}$ is in regime $i$, with unconditional probability $p_{i}$, for $i=1,2$, where $\sum_{i=1}^{2} p_{i}=1$. Accordingly,

$$
w_{t}= \begin{cases}w_{t, 1} \text { if } w_{t} \text { is in regime } 1 \text { with probability } p_{1}, \text { where } d w_{t, 1}=\mu_{1} d t+\sigma_{1} d B_{w} \\ w_{t, 2} \text { if } w_{t} \text { is in regime } 2 \text { with probability } p_{2}, \text { where } d w_{t, 2}=\mu_{2} d t+\sigma_{2} d B_{w}\end{cases}
$$

where $\mu_{i}$ and $\sigma_{i}$ are the mean and volatility of the Brownian motion in regime $i$, and $d B_{w}$ is the increment of the Wiener process. The step sizes of the regimes can be determined by Equation (6.15):

$$
\hat{\Delta}_{i}=\sqrt{\mu_{i}^{2}(\Delta t)^{2}+\sigma_{i}^{2} \Delta t}, \text { for } i=1,2 \text {. }
$$


As discussed in Section 6.3.1, a pentanominal lattice for the two-state regime-switching model is developed by adjusting the step size of one regime so that the step sizes of two regimes have a 1:2 ratio. The branch probabilities are then obtained by matching the moments of the lattice to the moments of the distributions and given by Equations (6.16) to (6.18) (Wahab and Lee 2009):

$$
\begin{gathered}
\wp_{\Delta_{j}, u}=\frac{1}{2}\left[\frac{\Delta_{j}^{2}}{\left(j \frac{\Delta_{i}}{i}\right)^{2}}+\frac{\mu_{j} \Delta t}{\left(j \frac{\Delta_{i}}{i}\right)}\right], \\
\wp_{\Delta_{j}, d}=\frac{1}{2}\left[\frac{\Delta_{j}^{2}}{\left(j \frac{\Delta_{i}}{i}\right)^{2}}-\frac{\mu_{j} \Delta t}{\left(j \frac{\Delta_{i}}{i}\right)}\right], \\
\wp_{\Delta_{j}, m}=1-\wp_{\Delta_{j}, u}-\wp_{\Delta_{j}, d} .
\end{gathered}
$$

Finally, the same three-step procedure presented in Section 6.3.1 is used to calculate the expected HDD or CDD using this lattice.

\subsubsection{Model 3: With two mean-reverting processes}

In this two-state regime-switching model, where each regime is governed by a mean-reverting process, the stochastic component, $w_{t}$, of the temperature can be represented by $w_{t, i}$, if $w_{t}$ is in regime $i$, with unconditional probability $p_{i}$, for $i=1,2$, where $\sum_{i=1}^{2} p_{i}=1$. Accordingly,

$w_{t}=\left\{\begin{array}{l}w_{t, 1} \text { if } z_{t} \text { is in regime } 1 \text { with probability } p_{1}, \text { where } d w_{t, 1}=\kappa_{w}\left(\bar{w}-w_{t, 1}\right) d t+\sigma_{1} d B_{w} \\ w_{t, 2} \text { if } w_{t} \text { is in regime } 2 \text { with probability } p_{2}, \text { where } d w_{t, 2}=\kappa_{w}\left(\bar{w}-w_{t, 2}\right) d t+\sigma_{2} d B_{w}\end{array}\right.$

where $\kappa_{w}$ is the speed and $\bar{w}$ is the mean of the mean-reverting process that remain the same in each regime, but volatilities of the mean-reverting processes, $\sigma_{1}$ and $\sigma_{2}$ switches between regimes or states, and $d B_{w}$ is the increment of the Wiener process.

Following the three-step procedure described in Section 6.3.1, a pentanomial lattice is constructed for which the step size of each regime is calculated by Equation (6.4). The branch probabilities for each lattice are determined by Equations (6.7) to (6.9). The expected HDD is calculated by Equation (6.13).

\subsubsection{Model 4: With a single mean-reverting process}

The binomial lattice presented in Nelson and Ramaswamy (1990) is applied to model the stochastic component, $w_{t}$, of the temperature by a mean-reverting process. The step size is defined by Equation (6.4). At any node, $w_{t}$ may jump up or down with the corresponding branch probabilities 
given by Equations (6.20) and (6.21):

$$
\begin{gathered}
\wp_{\Delta_{j}, u}= \begin{cases}\frac{1}{2}+\sqrt{\Delta t} \kappa_{w}\left(\bar{w}-w_{t}\right) / 2 \sigma & \text { if } 0 \leq \frac{1}{2}+\sqrt{\Delta t} \kappa_{w}\left(\bar{w}-w_{t}\right) / 2 \sigma \leq 1 \\
0, & \text { if } \frac{1}{2}+\sqrt{\Delta t} \kappa_{w}\left(\bar{w}-w_{t}\right) / 2 \sigma<0 \\
1, & \text { otherwise }\end{cases} \\
\wp_{\Delta_{j}, d}=1-\wp_{\Delta_{j}, u} .
\end{gathered}
$$

Using this binomial lattice, the same three-step procedure as presented in Section 6.3.1 is utilized to calculate the expected HDD or CDD. However, in the binomial lattice, the recursive function of the dynamic programming approach is modified as follows:

$$
\mathrm{HDD}_{t}=\max \left(0, \bar{Q}-Q_{t}\right)+\left(\mathbf{E}\left[\mathrm{HDD}_{t+1}\right]\right)
$$

\subsection{Numerical examples}

This section discusses the implementation of the models developed using numerical examples. To illustrate details of these models, four-period (four-day) lattices are presented as examples for Model 1. Since Model 2 and Model 3 are also two-state regime-switching models, they follow the same procedure and steps illustrated for Model 1. Section 6.4.4 shows the lattice construction and HDD calculations presented for Model 4, which is based on a single-regime approach.

\subsubsection{Model 1: With a mean-reverting process and a Brownian motion}

For the model described in Section 6.3.1, the regime parameters are obtained by discretizing Equation (6.3) for regime 1 as a first order autoregressive $\operatorname{AR}(1)$ form of $\Delta w_{t, 1}=\alpha_{1}+\varrho_{1} w_{t-1}+\epsilon_{t, 1}$ and regime 2 as a regressive form of $\Delta w_{t, 2}=\alpha_{2}+\epsilon_{t, 2}$, where $\alpha_{1}$ and $\alpha_{2}$ are the intercepts, $\varrho_{1}$ is the slope or the first order coefficient of $\operatorname{AR}(1), \epsilon_{t, 1} \sim \mathrm{N}\left(0, \sigma_{1}\right)$ and $\epsilon_{t, 2} \sim \mathrm{N}\left(0, \sigma_{2}\right)$ (Alexander 2008). Table 6.2 shows the parameters obtained that are estimated using the log-likelihood function maximized by the BFGS method (Brooks 2009).

Accordingly, the speed, $\kappa_{w}$, and the mean, $\bar{w}$, of regime 1 are calculated respectively by using the relationships between the continuous time mean-reverting process and discretized $\mathrm{AR}(1)$ as $b_{1}=\left(1+\kappa_{w}\right)^{-1}$ and $\alpha_{1}=\left(\bar{w} \kappa_{w}\right)\left(1+\kappa_{w}\right)^{-1}$ (Alexander 2008). The intercept $\alpha_{2}$ is interpreted as the mean of regime 2 (Brooks 2008). These parameters are given in Table 6.3. 
Table 6.2: Estimated regime coefficients for Model 1

\begin{tabular}{c|c|c|c|c}
\hline Variable & Coefficient & Standard error & T-statistic & $\mathrm{p}$-value \\
\hline$\gamma_{11}$ & 0.90 & 0.07 & 12.22 & 0.00 \\
$\gamma_{22}$ & 0.79 & 0.20 & 4.03 & 0.00 \\
$\alpha_{1}$ & -0.18 & 0.18 & -0.99 & 0.32 \\
$\varrho_{1}$ & -0.01 & 0.03 & -0.42 & 0.67 \\
$\alpha_{2}$ & 0.35 & 0.36 & 0.96 & 0.33 \\
$\sigma_{1}$ & 3.80 & 0.17 & 22.21 & 0.00 \\
$\sigma_{2}$ & 1.85 & 0.49 & 3.77 & 0.00 \\
\hline
\end{tabular}

Table 6.3: Regime parameters for Model 1

\begin{tabular}{c|c|c|c}
\hline Regime 1 & Value & Regime 2 & Value \\
\hline$\kappa_{w}$ & 99.00 & - & - \\
$\bar{w}$ & -0.18 & $\mu_{2}$ & 0.35 \\
$\sigma_{1}$ & 3.80 & $\sigma_{2}$ & 1.85 \\
\hline
\end{tabular}

To construct the corresponding lattice, the step sizes $\hat{\Delta}_{1}=3.80$ and $\hat{\Delta}_{2}=1.88$ are obtained from Equations (6.4) and (6.5), respectively. According to Section 6.3.1, since $\hat{\Delta}_{2}$ is less than half of $\hat{\Delta}_{1}, \hat{\Delta}_{2}$ is increased from 1.88 to 1.90 (i.e., half of the step size of 3.80 of regime 1 ) to efficiently merge the nodes of the lattice. The lattice expands in the forward direction with an increase in the number of nodes in accordance with $(4 t-3)$, where $t$ is the number of periods in the lattice.

The branch probabilities for the mean-reverting process (regime 1) are obtained using Equations (6.7) to (6.9). Since the step-size of this regime is unchanged, the middle branch probability is zero. Table 6.4 shows the other two branch probabilities of nodes from A to F in Figure 6.3.

Table 6.4: Branch probabilities for the mean-reverting process in Model 1

\begin{tabular}{c|c|c}
\hline Node & Upward & Downward \\
\hline A & 0.44 & 0.56 \\
B & 0.31 & 0.69 \\
C & 0.38 & 0.62 \\
D & 0.44 & 0.56 \\
E & 0.51 & 0.48 \\
F & 0.58 & 0.42 \\
\hline
\end{tabular}

The upward, middle, and downward branch probabilities for the Brownian motion (regime 2) are calculated as 0.05, 0.94, and 0.01, respectively, using Equations (6.10) to (6.12). Following the three-step procedure described in Section 6.3.1, Figure 6.3(a) shows a four-day pentanomial lattice for $w_{t}$ for the month of December, 2008. If $w_{t}$ is in regime 1 at node $\mathrm{A}$, it can stay in regime 1 (nodes $\mathrm{B}$ and F) or switch to regime 2 (i.e., nodes $\mathrm{C}, \mathrm{D}$, and E) in the next period. Similarly, if $w_{t}$ 
is in regime 2 at node $\mathrm{A}$, it can stay in regime 2 or switch to regime 1 in the next period. The lattice is initialized with $w_{t}=1.34$ on December 1 st. In the next period, the values of $w_{t}$ at nodes $\mathrm{B}$ and $\mathrm{C}$ are $1.34+2 \times 1.90=5.14$ and $1.34+1.90=3.24$ respectively. The temperature is now retrieved by adding corresponding $S_{t}=-0.04,-0.23,-0.42$, and -0.60 of the 1 st, 2 nd, 3rd and 4 th of December. The temperature on December 1 st is retrieved as $w_{t}+S_{t}=1.34+(-0.04)=1.3$ at node A in Figure 6.3(b). In the next day, the temperature at node B is obtained as: $5.14+(-0.23)=4.91$ and at node $\mathrm{C}$ as: $3.24+(-0.23)=3.01$.

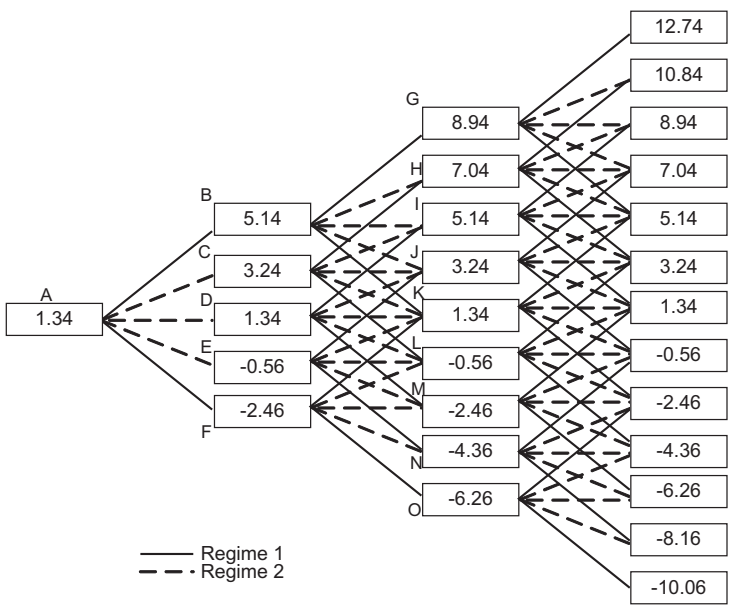

(a)

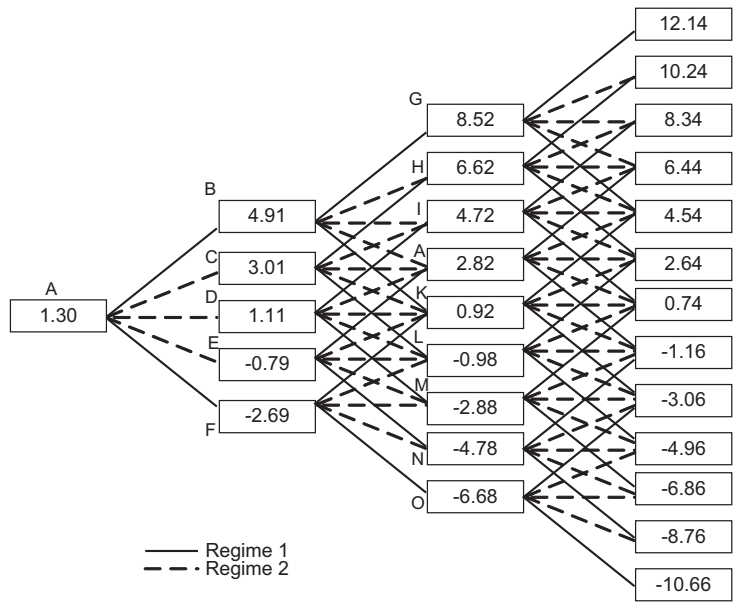

(b)

Figure 6.3: (a) A pentanomial lattice (for a mean-reverting process and a Brownian motion) for the stochastic component of temperature. (b) The corresponding pentanomial lattice for the behavior of temperature.

The value of HDD is calculated as $\max (0,18-12.14)=5.86$ at the topmost node in the last period of Figure 6.4. Applying Equation (6.13), the expected HDD is now calculated, for example, in period 2 of Figure 6.4 , at node B, as:

$$
\begin{aligned}
43.30 & =\max (0,18-(4.91) \\
& +0.90 \times(0.31 \times 21.38+0.0 \times 28.05+0.69 \times 34.72) \\
& +0.10 \times(0.05 \times 23.28+0.94 \times 26.98+0.01 \times 30.67) \\
40.71 & =\max (0,18-(4.91)) \\
& +0.21 \times(0.31 \times 21.38+0.0 \times 28.05+0.69 \times 34.72) \\
& +0.79 \times(0.05 \times 23.28+0.94 \times 26.98+0.01 \times 30.67)
\end{aligned}
$$

Working backwards from day 4 to day 1, at the root node of Figure 6.4, the expected HDD for 
regimes 1 and 2 are 69.74 and 68.27, respectively. If the unconditional regime probabilities are considered to be the initial regime, the expected HDD is $0.67 \times 69.74+0.33 \times 68.27=69.25$.

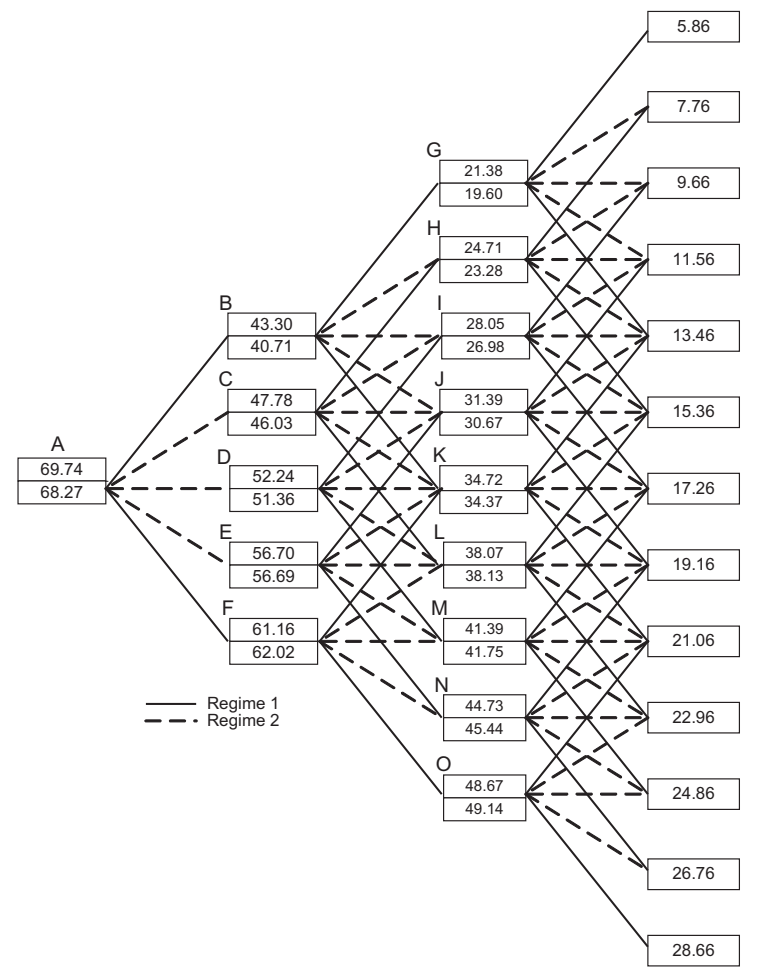

Figure 6.4: A pentanomial lattice (for a mean-reverting process and a Brownian motion) for the expected HDD.

\subsubsection{Model 2: With two Brownian motions}

For the model described in Section 6.3.2, the regime parameters are estimated by discretizing Equation (6.14) for regime 1 and regime 2, respectively, in the form of $\Delta w_{t, 1}=\alpha_{1}+\epsilon_{t, 1}$ and $\Delta w_{t, 2}=\alpha_{2}+\epsilon_{t, 2}$, where $c_{1}$ and $c_{2}$ are the means; and $\epsilon_{t, 1} \sim \mathrm{N}\left(0, \sigma_{1}\right)$ and $\epsilon_{t, 2} \sim \mathrm{N}\left(0, \sigma_{2}\right)$. These parameters based on daily temperature data are shown in Table 6.5.

Table 6.5: Estimated regime coefficients for Model 2

\begin{tabular}{c|c|c|c|c}
\hline Variable & Coefficient & Standard error & T-statistic & $\mathrm{p}$-value \\
\hline$\gamma_{11}$ & 0.79 & 0.20 & 3.99 & 0.00 \\
$\gamma_{22}$ & 0.90 & 0.07 & 12.26 & 0.00 \\
$\alpha_{1}$ & 0.34 & 0.37 & 0.93 & 0.35 \\
$\alpha_{2}$ & -0.18 & 0.18 & -1.02 & 0.31 \\
$\sigma_{1}$ & 1.85 & 0.52 & 3.55 & 0.00 \\
$\sigma_{2}$ & 3.80 & 0.19 & 19.50 & 0.00 \\
\hline
\end{tabular}

To construct the corresponding lattice, $\hat{\Delta}_{1}=1.88$ and $\hat{\Delta}_{2}=3.81$ are calculated by using Equation (6.15). In order to merge the nodes efficiently, $\hat{\Delta}_{1}$ is increased from 1.88 to 1.905 , which 
is half of $\hat{\Delta}_{2}$. The branch probabilities are computed using Equations (6.16) to (6.18). Regime 1 has probabilities of 0.58 (upward), 0.02 (middle), and 0.40 (downward); and Regime 2 has probabilities of 0.47 (upward), 0.0 (middle) and 0.53 (downward). The three-step procedure described in Section 6.3.1 is applied to construct the lattices and to calculate the expected HDD.

\subsubsection{Model 3: With two mean-reverting processes}

For the model described in Section 6.3.3, the parameters are obtained by discretizing Equation (6.19) for regime 1 and regime 2 respectively in the form of $\mathrm{AR}(1)$ as $\Delta w_{t, 1}=\alpha+\varrho w_{t-1}+\epsilon_{t, 1}$ and $\Delta w_{t, 2}=\alpha+\varrho w_{t-1}+\epsilon_{t, 2}$, where $\alpha$ is the intercept, $\varrho$ is the slope or the first order coefficient of $\operatorname{AR}(1), \epsilon_{t, 1} \sim \mathrm{N}\left(0, \sigma_{1}\right)$ and $\epsilon_{t, 2} \sim \mathrm{N}\left(0, \sigma_{2}\right)$ (Alexander 2008). It is found that $\alpha=-0.008$ and $\varrho=0.002$. From the relationship between the mean-reverting process given in Equation (6.19) and the discretized $\operatorname{AR}(1)$, the following relationship can be written: $\varrho=\left(1+\kappa_{w}\right)^{-1}$ and $\alpha=$ $\left(\bar{w} \kappa_{w}\right)\left(1+\kappa_{w}\right)^{-1}$ (Alexander 2008). Consequently, it is found that $\kappa_{w}=457.72$ and $\bar{w}=-0.008$. The regime parameters are given in Table 6.6.

Table 6.6: Regime parameters for Model 3

\begin{tabular}{c|c|c|c|c}
\hline Variable & Coefficient & Standard error & T-statistic & $\mathrm{p}$-value \\
\hline$\gamma_{11}$ & 0.94 & 0.04 & 22.21 & 0.00 \\
$\gamma_{22}$ & 0.92 & 0.20 & 4.61 & 0.00 \\
$\sigma_{1}$ & 3.90 & 0.53 & 7.34 & 0.00 \\
$\sigma_{2}$ & 2.28 & 1.13 & 2.01 & 0.04 \\
\hline
\end{tabular}

The calculation of step size and the construction of lattices for this model are the same as described for Model 1. The step sizes, $\hat{\Delta}_{1}=3.90$ and $\hat{\Delta}_{2}=2.28$, are obtained from Equation (6.4). The step size $\hat{\Delta}_{1}=3.90$ is then adjusted to 4.56 using Equation (6.6). The branch probabilities are obtained from Equations (6.7) to (6.9).

\subsubsection{Model 4: With a single mean-reverting process}

For the model described in Section 6.3.4, the parameters are obtained using the least-square first order autoregression method. The parameters are $\kappa_{w}=0.28, \bar{w}=-0.02$, and $\sigma=3.05$. The branch probabilities of the binomial lattice are obtained by using Equations (6.20) and (6.21).

Node A in Figure 6.5(a) is initialized with the same value of 1.34 similar to node A in Figure 6.3. The value at node $\mathrm{B}$ in the next level is $1.34+3.05=4.39$ and the value at node $\mathrm{C}$ is $1.34-3.05=$ -1.71. Figure $6.5(\mathrm{~b})$ is the lattice for temperature built by adding the corresponding seasonal component, $S_{t}$, with $w_{t}$. For example, the value at node A in Figure $6.5(\mathrm{~b})$ is $1.34+(-0.04)=1.30$. Table 6.7 presents the branch probabilities for the first three periods of the binomial lattices shown 
in Figure 6.5.

Table 6.7: Branch probabilities of the binomial lattice

\begin{tabular}{c|c|c}
\hline Node & Upward & Downward \\
\hline A & 0.4984 & 0.5016 \\
B & 0.4980 & 0.5020 \\
C & 0.4988 & 0.5012 \\
D & 0.4976 & 0.5024 \\
E & 0.4984 & 0.5016 \\
F & 0.4992 & 0.5008 \\
\hline
\end{tabular}

The expected HDD, as shown in Figure 6.6, is calculated using Equation (6.22). Working backwards from period 4 to period 1, at the root node of Figure 6.6, the expected HDD is 68.00.

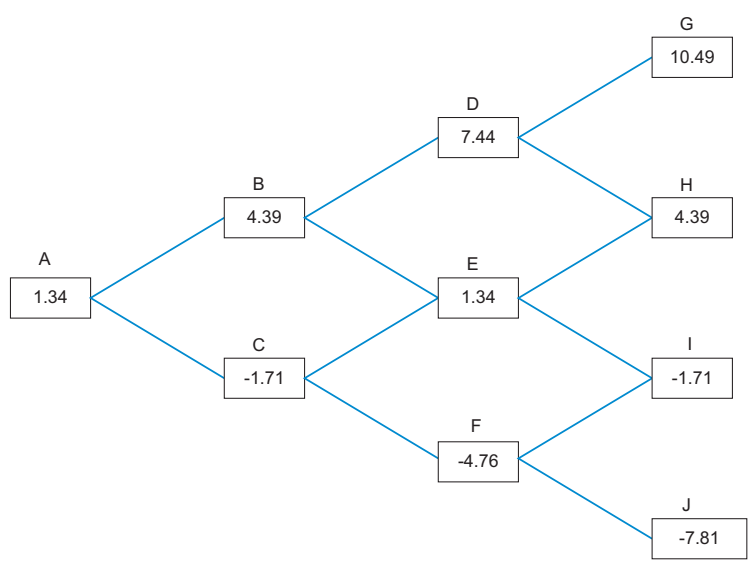

(a)

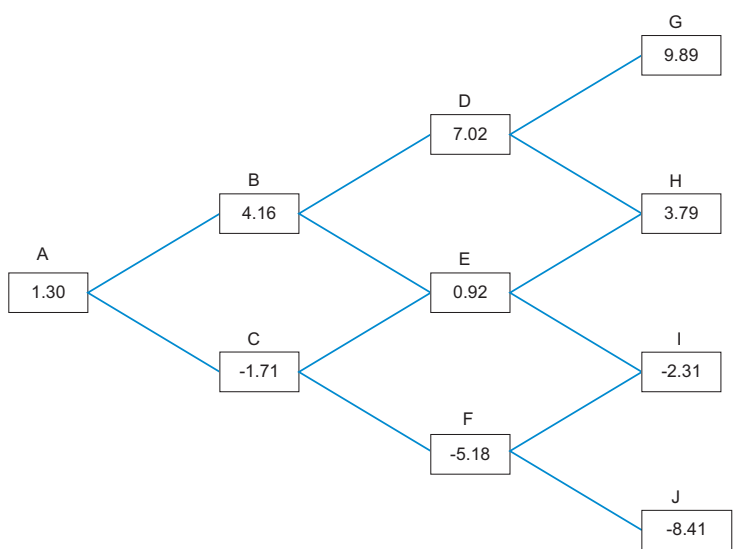

(b)

Figure 6.5: (a) A binomial lattice for the stochastic component of temperature. (b) The corresponding binomial lattice for the temperature.

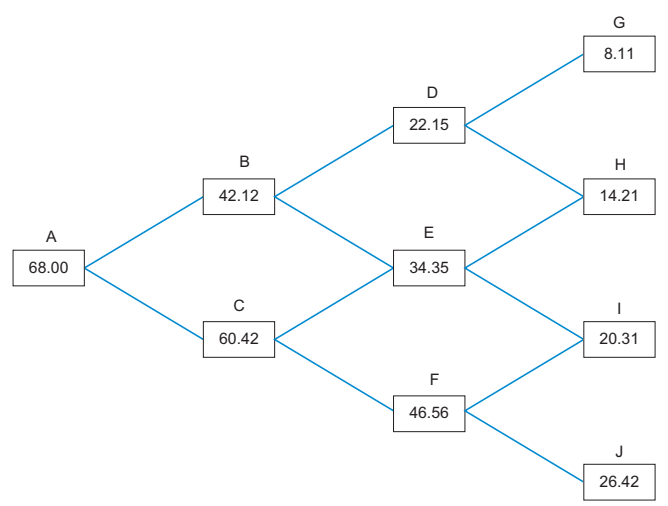

Figure 6.6: A binomial lattice for the expected HDD. 


\subsubsection{Comparison of the models}

The expected heating degree days (HDD) indices from the models are compared with the actual HDD indices over the period of 2005 to 2008. At CME, for the city of Toronto, Canada, HDD months are from January to April and from October to December; and CDD months are from April to October. Figure 6.7 shows the comparison of the expected monthly average HDD with the actual monthly average HDD. It is evident from the figure that for most of the HDD months, for example, for the months of January, March, April, and October, the expected monthly average HDDs obtained from Model 1 lies more closely with the actual monthly average HDD.

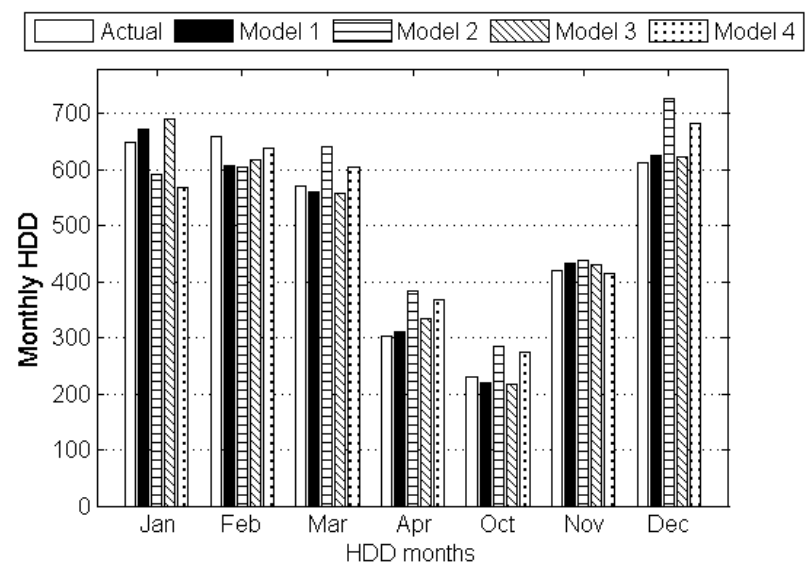

Figure 6.7: Comparison of the actual monthly HDD with the expected monthly HDD.

Figure 6.8 shows the comparison of CDD. Again, the expected monthly average CDD obtained from Model 1 is observed to be very close to the actual monthly average CDD for all CDD months. Therefore, it can be concluded that for both HDD and CDD months, Model 1, which is a twostate regime-switching model with a mean-reverting process and a Brownian motion, estimates the expected monthly HDD and CDD more closely to the actual HDD and CDD than the other three models. Considering unconditional probabilities for this model, it is found that there is a $68 \%\left(\approx \frac{1-0.79}{1+0.21-0.90}\right)$ probability of being in the mean-reverting regime. Consequently, it can be inferred that the temperature tends to stay in a base regime of mean-reverting process. A deviation from this regime prompted by a Brownian motion is relatively short lived and brought back to the base regime by a mean-reverting process. It is also observed that January, February, March and December are prominent HDD months, while June, July and August are prominent CDD months for the city of Toronto, Canada.

Furthermore, as there are seasonal HDD options (e.g., more than one-month options), Figure 6.9 and Figure 6.10 show the comparison of the actual seasonal HDD (CDD) with the expected seasonal HDD (CDD) obtained using Model 1. All the calculations are carried out with a market 


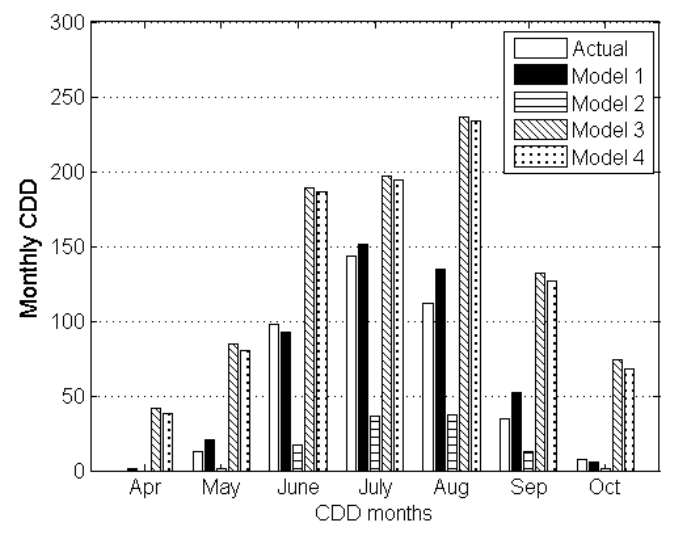

Figure 6.8: Comparison of the actual monthly CDD with the expected monthly CDD.

price of risk, $\lambda=0.10$. The observation from Figure 6.9 and Figure 6.10 also shows that while

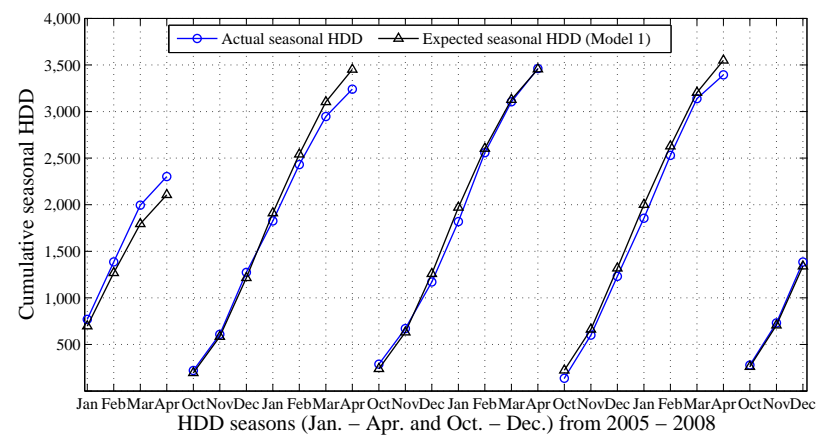

Figure 6.9: Comparison of the actual seasonal HDD with the expected seasonal HDD.

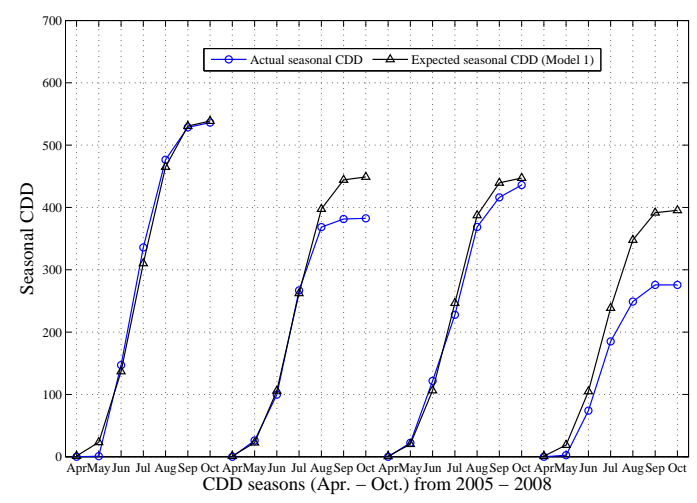

Figure 6.10: Comparison of the actual seasonal CDD with the expected seasonal CDD.

the actual seasonal HDDs are in the thousands, the actual seasonal CDDs are in the hundreds, suggesting more heating requirements in Toronto, Canada, than cooling requirements. Another point is that the average daily temperatures of CDD months - from April to October - are $6.3^{\circ} \mathrm{C}$, 
$12.9^{\circ} \mathrm{C}, 17.8^{\circ} \mathrm{C}, 20.8^{\circ} \mathrm{C}, 19.9^{\circ} \mathrm{C}, 15.3^{\circ} \mathrm{C}$, and $8.9^{\circ} \mathrm{C}$, respectively (Environment Canada 2009). It is observed that the daily average temperatures of only July and August are higher than $18^{0} \mathrm{C}$, above which CDD is counted and accumulated. Hence, in Figure 6.10, the seasonal CDD curves begin flatly during April to May and end flatly during September to October. Since the shoulder months of April and October have little effects on seasonal CDD, the CME could reconsider the CDD months for Toronto, Canada, by excluding the months of April and October.

\subsubsection{Option pricing}

The challenge of pricing temperature-based weather derivatives lies on the appropriate modeling of dynamic behavior of temperature. Pricing mechanisms of weather derivatives in the literature range from the marginal utility technique (e.g., Davis 2001) to the simulation approach based on the Ornstein-Uhlenbeck process (e.g., Alaton et al. 2002) to an autoregressive temperature modeling approach (e.g., Benth et al. 2007). The resulting option prices obtained here are based on regimeswitching modeling of temperature dynamics and are dependent on the choice of the strike heating degree day, $K$, tick size, $\tau$, and market price of risk, $\lambda$. An important point to note, as observed from Figure 6.7, is that the expected HDD obtained from Model 1 is very close to the actual HDD. Therefore, Model 1 is used in the valuation of the option price. To price a standard HDD call option, the following payoff function is used:

$$
\varphi=e^{-r t} \tau[\max (\mathrm{HDD}-K, 0)]
$$

where HDD is the expected HDD during the contract period $[0, t], r$ is the risk-free interest rate, $\tau$ is the ticksize, and $K$ is the strike HDD.

In the following examples, $\tau=\$ 20$ per HDD; $r=5 \%$; the contract period for the monthly option price is from December 1 to December 31, 2008; and the seasonal option prices are calculated for HDD seasons (October, November, and December). For example, the expected HDD (from Model 1) for the contract period for the month of December 2008 is 632.28 and the option price for $K=620$ is, therefore:

$$
\varphi=e^{-(0.05)\left(\frac{31}{365}\right)} \times 20 \times[\max (632.28-620,0)]=\$ 244.56
$$

Figure 6.11(a) shows the variation of monthly option prices over a range of the strike HDD. As the strike HDD, $K$, increases from 610 to 650 , the option price decreases until the strike HDD equals to the expected HDD at 632.28 and then falls to zero. Figure 6.11(b) shows the variation in seasonal (one month of October, two months of October and November, and three months of 
October, November, and December of 2008) option prices over a range of strike HDD from 100 to 1400. It is evident from Figure 6.11(b) that as more months are included into the contract, the more premium one has to pay.

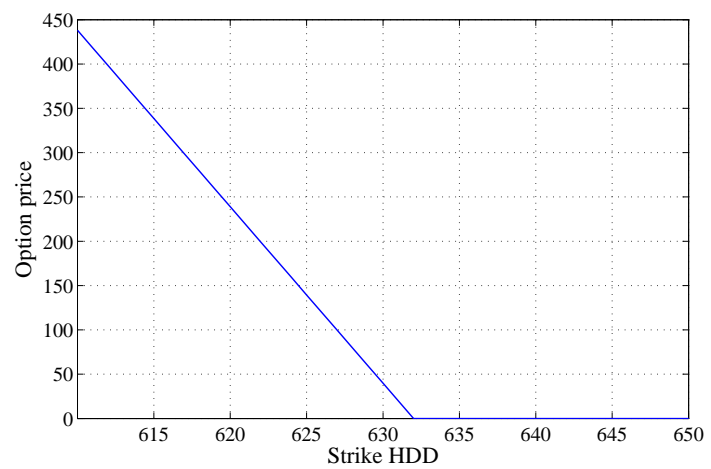

(a)

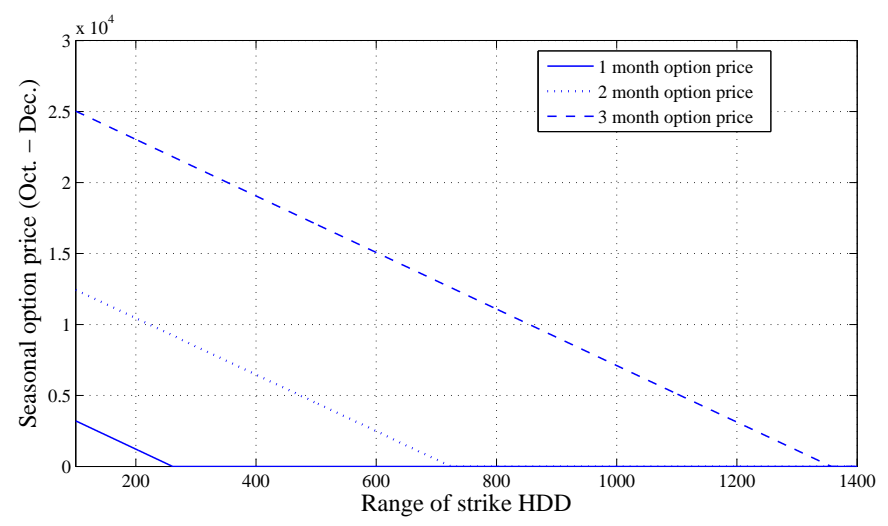

(b)

Figure 6.11: (a) Monthly and (b) seasonal option prices for a range of strike HDD.

As mentioned earlier in Section 6.4.5 that a value of $\lambda=0.10$ is assumed for all previous calculations. A sensitivity analysis is carried out to show the effect of market price of risk, $\lambda$, on option price by varying $\lambda$ between 0 and 0.30 . Figure 6.12 shows that the monthly option price increases with the increase in $\lambda$. This is consistent with the finding of Cao and Wei (2004) that the market price of risk has a significant role for temperature derivatives. 


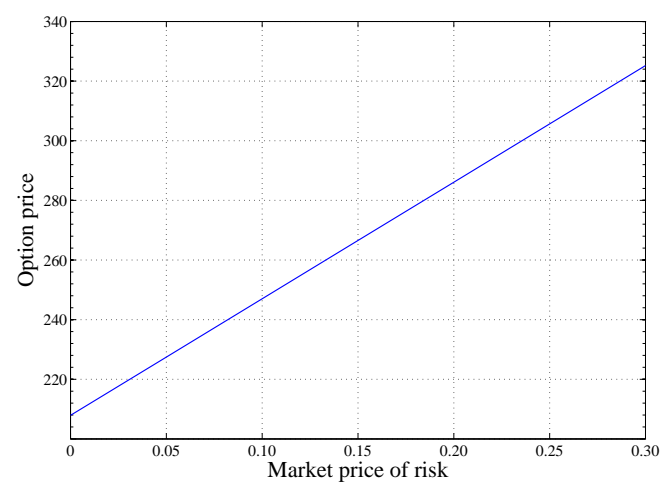

Figure 6.12: Option prices at different market prices of risk. 


\subsection{Summary}

Modeling the temperature dynamics is essential for pricing temperature-based weather options. This thesis discusses the rationale behind the regime-switching behavior of temperature and examines regime-switching approaches to model the temperature dynamics of the city of Toronto, Canada. The study finds that there exists a regime-switching behavior in Toronto's temperature induced by abrupt changes in the city's temperature. Three two-state regime-switching models and one single regime model have been developed. The first model assumes that one regime is governed by a mean-reverting process and the other by a Brownian motion. The second model is based on two regimes, each of which is governed by a Brownian motion. The third model assumes that each regime is governed by a mean-reverting process in which the mean and speed of the mean-reversion remain the same, but only the volatility switches between the states. In the fourth model, the temperature dynamics are governed by a single mean-reverting process. Each model is used to calculate the expected HDD and CDD for a monthly contract period. Results show that the two-state regime-switching model with a mean-reverting process and a Brownian motion determines both HDD and CDD more closely to the actual HDD and CDD than those obtained by the other three models. Using this model, monthly and seasonal call options are then priced under the martingale probability approach by adjusting the process drift to the risk-neutral equivalent drift considering the risk premium. 


\section{Chapter 7}

\section{Conclusion and Future Work}

This thesis is centered around the valuation models. Stricter environmental regulations entail power plant owners to consider the cost of emission in assessing the value of a power plant. One of the objectives of the research is to assess the value of a natural gas-fired power plant under carbon emission restrictions in order to observe the effect of the presence and absence of carbon allowance prices. Moreover, unlike existing valuation models, the power plant is assumed to have multiple turbines with different carbon emission rates, generation capacities, and input-output characteristics. This thesis investigates how the operational flexibility of switching among turbines in accordance with the correlated price movements affects the power plant value in presence and absence of carbon allowance prices. Prices of electricity and natural gas are assumed to follow mean-reverting processes. Results demonstrate that the value of a power plant is reduced due to $\mathrm{CO}_{2}$ allowance cost, while operational flexibility of switching adds value to the power plant. To examine the modeling effect, $\mathrm{CO}_{2}$ allowance prices are modeled as both a mean-reverting process and a geometric Brownian motion, in case of the clean spark spread option based valuation. A comparison of these two cases shows that modeling $\mathrm{CO}_{2}$ prices as a Brownian motion overvalues the power plant in comparison to when $\mathrm{CO}_{2}$ allowance prices are assumed to follow a meanreverting process. It is also observed that both spark and clean spark spread option based values of the power plant increases with an increase in the electricity price volatility. However, an increase in the volatility of natural gas and $\mathrm{CO}_{2}$ prices decreases the expected power plant value. With regard to sensitivity analyses on the mean-reverting speeds of prices show that an increase in the mean-reverting speed of electricity prices reduces the value of the power plant, whereas an increase in the mean-reverting speed of natural gas prices tends to increase the value of the power plant.

This thesis also presents a model that integrates weather option into the spark spread based valuation model. The motivation behind this approach is to exercise the temperature-based put option in an anticipation of a not-so-cold winter condition when heating degree days fall below the 
strike heating degree day. A three variable lattice is utilized for modeling the correlated behavior of electricity and natural gas prices, and temperature. The lattice discretizes the movements of these variables, all of which are assumed to follow mean-reverting processes. Depending on the movements of prices and temperature at different states, the proposed model finds the optimal number of weather contracts to buy. Pricing of the weather option is determined based on the mean-reverting behavior of temperature. Results demonstrate that when the winter is cooler, the expected spark spread option based value of the power plant is higher. When the winter is warmer, the expected spark spread option based value tends to drop. However, exercising the weather put option in case of a warm winter adds value to the spark spread option based valuation. The expected value of the power plant then tends to increase.

Pricing weather option is an important issue in integrating weather option into spark spread option. This thesis also analyzes the temperature dynamics with an aim to valuing weather option. Four models are developed. Three of these are two-state Markov regime-switching models and the other is a single-regime model. Different underlying processes generate different regimes or states for the stochastic component of temperature. In the first model, one regime is governed by a mean-reverting process and the other regime is governed by a Brownian motion. In the second model, each regime is governed by a Brownian motion. In the third model, each regime is governed by a mean-reverting process. The fourth model is a single-regime model, where the temperature dynamics are governed by a single mean-reverting process. All four models are utilized to determine the expected heating degree days (HDD) and cooling degree days (CDD), which play a crucial role in the valuation of weather options. Results demonstrate that in calculating monthly degree days, the first model captures the temperature dynamics more accurately than the other three models. The monthly call option prices are then calculated based on this model for a range of strike HDD.

\subsection{Summary of contributions}

The summary of the contributions of this thesis can be underlined in three perspectives:

(i) In the existing literature, the valuation models are mostly based on the spark spread option and power plants are assumed to posses a single turbine. As voices for stricter environmental regulations are increasing all over the world, in this thesis, the carbon emission cost is included in the valuation model along with a more realistic assumption that a power plant possesses multiple turbines. These turbines are of different generation capacities and different emission and heat rates. With a single turbine, the operational flexibility arises from operating the turbine according to spark spread option (also known as 'cycling') against the scenario of operating the turbine irrespective of price movements throughout the planning period (Heydari 
and Siddiqui 2010; Abadie 2015). In this thesis, the operational flexibility of the power plant, therefore, arises from switching the turbines, all of which are in 'cycling' mode in response to price movements.

(ii) Integrating weather effect into the spark spread option based valuation model can be considered as another main research contribution. The motivation is to examine exercising weather put option in case of an unusual not-so-cold winter. A three variable lattice is utilized to model the correlated behavior of prices of electricity and natural gas and temperature. The number of contracts to long are determined such that the standard deviation of the total payoff is minimized.

(iii) With an aim to valuing weather option, a comparison of models which capture the temperature dynamics is also investigated. The thesis examines whether there exists a regime-switching behavior in temperature dynamics. Results demonstrate that a regime-switching model, in which one regime is governed by a mean-reverting process and the other is governed by a Brownian motion, captures the temperature dynamics more accurately in calculating degree days in case of the city of Toronto, Canada.

\subsection{Challenges, limitations and future work}

Like any research endeavor, this research also faces challenges and limitations. An Independent System Operator (ISO), also known as a System Controller (SC), decides when to connect which generators in the grid to supply power. The ISO gives priority according to the bid stack. However, many generators may have the same bid. Alternatively, a power plant operator may have several identical generators. The generators will then be allowed to connect one at a time by the ISO. Otherwise, there will be a shock in the grid, if all generators are connected to the grid all at once. In order to discourage the power surge in the grid, in the proposed model, a penalty cost could be imposed whenever the power plant attempts to switch to State 4 turning both turbines 'on' at the same time. The switching cost matrix given in Equation (4.17) could adapt this modification.

One of the challenges is to model the price processes rightly. "Energies are harder to model" (Pilipovic, 2007). Energy prices also show different characteristics at different markets. Modeling electricity prices, in the literature, ranges from different forms of mean-reverting processes to regime switching processes (Fiorenzani 2006; Weron 2007; Wahab et al. 2010). An augmented Dickey-Fuller test can be used to see whether the return on the commodity price, for example, $d x=\ln P_{t}-\ln P_{t-1}$, is likely to follow a mean-reverting process. Using the statistical MATLAB function '[h, pValue $]=$ adftest()' on the returns of prices used in this thesis show a 'p-value' of 
less than 0.05 , which is an indication that the price returns used in this theses are likely to follow mean-revering processes (Teetor 2011).

A large volume of trading in commodity markets occurs on Exchanges. Producers and consumers use futures prices, instead of spot prices. Spot prices are prices at which physical commodities are delivered 'immediately' or 'near immediately', say on the next day, at specified locations (Coulon 2013). The intricacies of physical shipment of commodities, for example, by pipelines, ships, or trucks, and the issues of storage, if the commodity is not immediately consumed, are entangled with spot prices. Therefore, applying the contingent claim analysis in valuing real assets requires the determination of the risk of the 'price', i.e., market price, which is hard to estimate (Abadie 2015). Futures price, on the other hand, are the prices free of the complications of physical delivery and does not require to estimate the risk of price. By using futures prices, the expected value of an asset can be determined by discounting at a risk-free rate. Electricity, natural gas and carbon prices are the futures prices taken from European Energy Exchange (EEX).

Extracting model parameters from the data is also an important aspect. This study uses the least square regression technique. Kalman filter could also be used in extracting process parameters. It is a recursive procedure that updates unobservable state variables (e.g., spot prices) expressed as a 'transition equation' based on the observations (e.g., futures prices) that depend on the state variables represented as a 'measurement equation' (Schwartz 1997; Schwartz and Smith 2000). However, Kalman filter has a relatively high implementation requirement (Abadie and Chamorro 2008b).

With regard to the locations of data in integrating spark spread and weather options, an explanation is in order. The data used for the prices of electricity and natural gas and for temperature are not from the same locations. Data accessibility is a major reason. Futures prices are purchased from the European Emission Exchange (EEX), while temperature data are collected from the website of Environment Canada. However, it is imperative to state that the purpose of the thesis is to develop a generic model that integrates weather and spark spread options. Since the model developed in this thesis is generic, any relevant data can be used to assess the value of a power plant. The prices of electricity and natural gas are converted to Canadian dollar (CAD) by using the corresponding exchange rates from Euro to CAD and are used as pxoyies for the prices in Canada. Applicability of the models is another issue - who could use the models? According to the convenience of the models, the power plant owners, investors, operators, prospective buyers or sellers are the target groups.

The current research can be extended in many ways. An interesting extension would be to develop a valuation model that considers stochastic processes for electricity, natural gas, and carbon allowance prices, along with temperature. How both carbon allowance prices and temperature affect 
the value of a power plant could be an issue of investigation. Developing a correlated four variable lattice will be a challenge. The computational burden would be another important issue.

As electricity prices often show spikes, the electricity prices can be modeled as a mean-reverting process with jumps or spikes or as a regime-switching behavior. By representing the correlated regime-switching behavior of temperature and electricity prices along with a mean-reverting behavior of natural gas prices, a valuation model that integrates spark spread and weather options could be an interesting future extension. The existing Markov regime-switching models can also be extended with additional flexibility by incorporating time-varying transition probabilities. Challenges would arise to model the correlated behavior of regime-switching along with a single regime mean-reverting process. Wahab and Lee (2009) develop a lattice approach that models multivariate correlated regime-switching processes. Merging a mean-reverting process into this model could be a possible mode of solution. However, compared to a geometric Brownian motion, modeling a mean-reverting process is more complicated with respect to determining the branch probabilities (Hahn and Dyer 2008).

Rather than purchasing carbon allowances, the power plant owner could invest in a longterm carbon capture and storage project. An assessment of the option value of either emitting or capturing the carbon in case of a gas-fired power plant would be another interesting research project. In case of a carbon capture and storage project, flues gases from the power plant, mainly $\mathrm{CO}_{2}$, is separated, and then transported via a pipeline to a storage site where it is injected for a suitable geological formation such as deep saline aquifer.

An extension of the current research could also be to assess both the value and the optimal time to replace a gas turbine having a higher carbon emission rate by a more efficient one with a lower carbon emission rate. Via a lattice approach, the decision can be made at each node whether it is suitable to abandon the old turbine or to invest for purchasing a new turbine with a lower carbon emission rate. 


\section{Appendix A}

\section{The Mean Reverting Process}

A commodity is usually a consumption good with little or no variation in quality between supply sources (Coulon 2013). Metals, agricultural products and energy fall into this category. Let $P_{t}$ be the price of a commodity. Then, assuming $x_{t}=\ln P_{t}$ follows a mean-reverting process and the process of $x_{t}$ can be expressed as follows:

$$
d x_{t}=\kappa\left(\bar{x}-x_{t}\right) d t+\sigma d B_{t},
$$

where $\kappa$ is the mean-reversion rate, $\bar{x}$ is the long-term average level, $d t$ is the time interval, $\sigma$ is the instantaneous volatility, and $B_{t}$ follows a standard Winner or Brownian process, the change of which, $\Delta B$, is normally distributed with mean, $\mathbf{E}(d B)=0$ and the linearly increasing variance, $\operatorname{Var}(d B)=d t$ (Trigeorgis 1996). Equation (A.1) implies that the instantaneous price $x_{t}$ reverts to the level $\bar{x}$ at the rate of $\kappa$. If $x_{t}$ is below the long-term average level $\bar{x}$, positive rate of reversion, $\kappa$ will induce the price to go up; if $x_{t}$ moves up, the negative rate of reversion will influence the price $x_{t}$ to move down to the average level. The process is also known as the Orenstein-Uhlenbeck process.

\section{A.1 The Mean and Variance of the Process}

- Apply Itô's lemma. Therefore, if $d x=a(x, t) d t+b(x, t) d B$ and $G$ is a function expressed as $G(x, t)$ then according to Itô's lemma (Hull 2002):

$$
d G=\left(\frac{\partial G}{\partial x} a+\frac{\partial G}{\partial t}+\frac{1}{2} \frac{\partial^{2} G}{\partial x^{2}} b^{2}\right) d t+\frac{\partial G}{\partial x} b d B
$$

- To find the derivative the stochastic process given by Equation (A.1), we assume that $G\left(x_{t}, t\right)=$ 
$x_{t} e^{\kappa t}$. Therefore, $\frac{\partial G}{\partial x}=e^{\kappa t} ; \frac{\partial G}{\partial t}=x_{t} \kappa e^{\kappa t}$; and $\frac{\partial^{2} G}{\partial x^{2}}=0$.

$$
\begin{aligned}
d G & =\left(e^{\kappa t} \kappa\left(\bar{x}-x_{t}\right)+\kappa x_{t} e^{\kappa t}+0\right) d t+e^{\kappa t} \sigma d B_{t} \\
d\left(x_{t} e^{\kappa t}\right) & =e^{\kappa t} \kappa\left(\left(\bar{x}-x_{t}\right)+x_{t}\right) d t+e^{\kappa t} \sigma d B_{t} \\
d\left(x_{t} e^{\kappa t}\right) & =\bar{x} \kappa e^{\kappa t} d t+\sigma e^{\kappa t} d B_{t}
\end{aligned}
$$

- Integrating Equation (A.3) from 0 to $t$, we have:

$$
\begin{aligned}
\int_{0}^{t} d\left(x_{t} e^{\kappa t}\right) & =\int_{0}^{t} \bar{x} \kappa e^{\kappa t} d t+\int_{0}^{t} \sigma e^{\kappa t} d B_{t} \\
x_{t} e^{\kappa t}-x_{0} e^{\kappa 0} & =\bar{x} \kappa \int_{0}^{t} e^{\kappa s} d s+\int_{0}^{t} \sigma e^{\kappa s} d B_{s} \\
x_{t} e^{\kappa t} & =x_{0}+\xi \kappa\left(\left[\frac{1}{\kappa} e^{\kappa s}\right]_{0}^{t}\right)+\int_{0}^{t} \sigma e^{\kappa s} d B_{s} \\
x_{t} e^{\kappa t} & =x_{0}+\xi \kappa\left(\frac{1}{\kappa} e^{\kappa t}-\frac{1}{\kappa} e^{\kappa 0}\right)+\int_{0}^{t} \sigma e^{\kappa s} d B_{s} \\
x_{t} e^{\kappa t} & =x_{0}+\bar{x}\left(e^{\kappa t}-1\right)+\int_{0}^{t} \sigma e^{\kappa s} d B_{s} \\
x_{t} & =\frac{x_{0}+\xi\left(e^{\kappa t}-1\right)}{e^{\kappa t}}+\frac{\int_{0}^{t} \sigma e^{\kappa s} d W_{s}}{e^{\kappa t}} \\
x_{t} & =x_{0} e^{-\kappa t}+\bar{x}\left(1-e^{-\kappa t}\right)+\int_{0}^{t} \sigma e^{\kappa(s-t)} d B_{s} .
\end{aligned}
$$

- Mean value: Given that $x_{0}$ is a constant, the conditional mean of $x_{t}$ is:

$$
\mathbf{E}\left(x_{t} \mid x_{0}\right)=e^{-\kappa t} x_{0}+\bar{x}\left(1-e^{-\kappa t}\right) .
$$

Equation (A.13) shows that $X_{0}$ decays exponentially, in absence of $\sigma$, at a rate of $\kappa(\kappa>0)$ and eventually reaches to a long-term mean $\bar{x}$.

- Variance:

$$
\begin{aligned}
& \operatorname{Var}\left(x_{t}\right)=\mathbf{E}\left[\left(x_{t}-\mathbf{E}\left(x_{t}\right)\right)^{2}\right] \\
& \operatorname{Var}\left(x_{t}\right)=\mathbf{E}\left[\left(\int_{0}^{t} \sigma e^{\kappa(s-t)} d B_{s}\right)^{2}\right]
\end{aligned}
$$

From the Itô isometry (Øksendal 2000):

$$
\mathbf{E}\left[\left(\int_{0}^{t} \sigma e^{\kappa(s-t)} d B_{s}\right)^{2}\right]=\int_{0}^{t}\left(\sigma e^{\kappa(s-t)}\right)^{2} d s .
$$


Therefore, given that $x_{0}$ is a constant, the variance is:

$$
\begin{aligned}
& \operatorname{Var}\left(x_{t} \mid x_{0}\right)=\int_{0}^{t} \sigma^{2} e^{2 \kappa(s-t)} d s \\
& \operatorname{Var}\left(x_{t} \mid x_{0}\right)=\frac{\sigma^{2}}{2 \kappa}\left(1-e^{-2 \kappa t}\right) .
\end{aligned}
$$

Equation (5.14) shows that as $t \rightarrow \infty$, the variance of the mean reverting process is bounded by the value of $\operatorname{Var}\left(x_{t} \mid x_{0}\right)=\sigma^{2} / 2 \kappa$, which is unlike the variance of the geometric Brownian motion that keeps increasing as $t \rightarrow \infty$.

As a result, the commodity price, $P_{t}$, is log-normally distributed with mean

$$
\begin{aligned}
& \mathbf{E}\left(P_{t} \mid x_{0}\right)=\exp \left\{\mathbf{E}\left(x_{t} \mid x_{0}\right)+\frac{1}{2} \operatorname{Var}\left(x_{t} \mid x_{0}\right)\right\} \\
& \mathbf{E}\left(P_{t} \mid x_{0}\right)=\exp \left\{e^{-\kappa t} x_{0}+\xi\left(1-e^{-\kappa t}\right)+\left(1-e^{-2 \kappa t}\right) \frac{\sigma^{2}}{4 \kappa}\right\} .
\end{aligned}
$$




\section{Appendix B}

\section{Building a Bivariate Lattice}

Assuming a correlated process as:

$$
\begin{aligned}
d x & =\nu_{x} d t+\sigma_{x} d B_{x} \\
d y & =\nu_{y} d t+\sigma_{y} d B_{y}
\end{aligned}
$$

The probabilities $\left(p_{u u}, p_{u d}, p_{d u}\right.$ and $\left.p_{d d}\right)$ and jump sizes $\left(\Delta_{x}\right.$ and $\left.\Delta_{y}\right)$ are chosen to match the mean and variances of the processes (Clewlow and Strickland 1998):

$$
\begin{aligned}
\mathbf{E}\left[\Delta_{x}\right] & =\left(p_{u u}+p_{u d}\right) \Delta_{x}-\left(p_{d u}+p_{d d}\right) \Delta_{x}=\nu_{x} \Delta t \\
\mathbf{E}\left[\left(\Delta_{x}\right)^{2}\right] & =\left(p_{u u}+p_{u d}\right)\left(\Delta_{x}\right)^{2}-\left(p_{d u}+p_{d d}\right)\left(\Delta_{x}\right)^{2}=\sigma_{x}^{2} \Delta t \\
\mathbf{E}\left[\Delta_{y}\right] & =\left(p_{u u}+p_{d u}\right) \Delta_{y}-\left(p_{u d}+p_{d d}\right) \Delta_{y}=\nu_{y} \Delta t \\
\mathbf{E}\left[\left(\Delta_{y}\right)^{2}\right] & =\left(p_{u u}+p_{d u}\right)\left(\Delta_{y}\right)^{2}-\left(p_{u d}+p_{d d}\right)\left(\Delta_{y}\right)^{2}=\sigma_{y}^{2} \Delta t \\
\mathbf{E}\left[\Delta_{x} \Delta_{y}\right] & =\left(p_{u u}-p_{u d}-p_{d u}-p_{d d}\right) \Delta_{x} \Delta_{y}=\rho \sigma_{x} \sigma_{y} \Delta t
\end{aligned}
$$

The probabilities must sum to one.

$$
p_{u u}+p_{u d}+p_{d u}+p_{d d}=1
$$


The solutions to the system of equations (Equations (B.3)-(B.8) are:

$$
\begin{aligned}
\Delta_{x} & =\sigma_{x} \sqrt{\Delta t} \\
\Delta_{y} & =\sigma_{y} \sqrt{\Delta t} \\
p_{u u} & =\frac{\Delta_{x} \Delta_{y}+\Delta_{y} \nu_{x} \Delta t+\Delta_{x} \nu_{y} \Delta t+\rho_{x y} \sigma_{x} \sigma_{y} \Delta t}{4 \Delta_{x} \Delta_{y}} \\
p_{u d} & =\frac{\Delta_{x} \Delta_{y}+\Delta_{y} \nu_{y} \Delta t-\Delta_{x} \nu_{y} \Delta t-\rho_{x y} \sigma_{x} \sigma_{y} \Delta t}{4 \Delta_{x} \Delta_{y}} \\
p_{d u} & =\frac{\Delta_{x} \Delta_{y}-\Delta_{y} \nu_{y} \Delta t+\Delta_{x} \nu_{y} \Delta t-\rho_{x y} \sigma_{x} \sigma_{y} \Delta t}{4 \Delta_{x} \Delta_{y}} \\
p_{d d} & =\frac{\Delta_{x} \Delta_{y}-\Delta_{y} \nu_{y} \Delta t-\Delta_{x} \nu_{y} \Delta t+\rho_{x y} \sigma_{x} \sigma_{y} \Delta t}{4 \Delta_{x} \Delta_{y}} .
\end{aligned}
$$




\section{Appendix C}

\section{Building a Trivariate Lattice}

Assuming a correlated process as:

$$
\begin{aligned}
d x & =\nu_{x} d t+\sigma_{x} d B_{x} \\
d y & =\nu_{y} d t+\sigma_{y} d B_{y} \\
d z & =\nu_{z} d t+\sigma_{z} d B_{z}
\end{aligned}
$$

Matching the second moment, we get:

$$
\begin{aligned}
& \mathbb{E}\left(\Delta x^{2}\right)=\left(p_{\text {uuu }}+p_{\text {uud }}+p_{\text {udu }}+p_{\text {udd }}\right)\left(\Delta x^{2}\right)+\left(p_{\text {duu }}+p_{\text {dud }}+p_{\text {ddu }}+p_{\text {ddd }}\right)\left(\Delta x^{2}\right), \\
& \mathbb{E}\left(\Delta x^{2}\right)=\Delta x^{2} .
\end{aligned}
$$

We have, $\Delta x^{2}=\sigma_{x}^{2} \Delta t+\nu_{x}^{2}(\Delta t)^{2}$. Since, $(\Delta t)^{2} \simeq 0$, we get $\Delta x^{2}=\sigma_{x}^{2} \Delta t$. Therefore, the increments for the processes are respectively:

$$
\begin{aligned}
& \Delta x=\sigma_{x} \sqrt{\Delta t} \\
& \Delta y=\sigma_{y} \sqrt{\Delta t} \\
& \Delta z=\sigma_{z} \sqrt{\Delta t} .
\end{aligned}
$$

Considering the observed correlation between natural gas and electricity prices, we get:

$$
\begin{aligned}
\mathbb{E}(\Delta x \Delta y) & =\left(p_{\text {uuu }}+p_{\text {uud }}-p_{u d u}-p_{u d d}-p_{d u u}-p_{d u d}+p_{d d u}+p_{d d d}\right)(\Delta x \Delta y) \\
& =\rho_{x y} \sigma_{x} \sigma_{y} \Delta t+\nu_{x} \nu_{y}(\Delta t)^{2} .
\end{aligned}
$$


Since, $(\Delta t)^{2} \simeq 0$, we have:

$$
p_{u u u}+p_{u u d}-p_{u d u}-p_{u d d}-p_{d u u}-p_{d u d}+p_{d d u}+p_{d d d}=\rho_{x y} .
$$

Similarly, we get:

$$
\begin{gathered}
p_{u u u}-p_{u u d}-p_{u d u}+p_{u d d}+p_{d u u}-p_{d u d}-p_{d d u}+p_{d d d}=\rho_{y z} \\
p_{u u u}-p_{u u d}-p_{u d u}+p_{u d d}+p_{d u u}-p_{d u d}-p_{d d u}+p_{d d d}=\rho_{z x} .
\end{gathered}
$$

Matching the first moment, we get:

$$
\mathbb{E}(\Delta x)=\left(p_{\text {uuu }}+p_{\text {uud }}+p_{\text {udu }}+p_{\text {udd }}-p_{\text {duu }}-p_{\text {dud }}-p_{d d u}-p_{d d d}\right)(\Delta x)=\nu_{x} \Delta t,
$$

Therefore, we have:

$$
p_{u u u}+p_{u u d}+p_{u d u}+p_{u d d}-p_{d u u}-p_{d u d}-p_{d d u}-p_{d d d}=\frac{\nu_{x} \Delta t}{\sigma_{x}} .
$$

Similarly, we get:

$$
\begin{aligned}
& p_{u u u}+p_{u u d}-p_{u d u}-p_{u d d}+p_{d u u}+p_{d u d}-p_{d d u}-p_{d d d}=\frac{\nu_{y} \Delta t}{\sigma_{y}} . \\
& p_{u u u}-p_{u u d}+p_{u d u}-p_{u d d}+p_{d u u}-p_{d u d}+p_{d d u}-p_{d d d}=\frac{\nu_{z} \Delta t}{\sigma_{z}} .
\end{aligned}
$$

The sum of probabilities should be one:

$$
p_{u u u}+p_{u u d}+p_{u d u}+p_{u d d}+p_{d u u}+p_{d u d}+p_{d d u}+p_{d d d}=1
$$


Solving Equations (C.5)-(C.16) by the method suggested by Boyle et al. (1989), branch probabilities can be obtained as:

$$
\begin{aligned}
& p_{u u u}=\frac{1}{8}\left[1+\rho_{x y}+\rho_{y z}+\rho_{z x}+\Delta t\left(\frac{\nu_{x}}{\sigma_{x}}+\frac{\nu_{y}}{\sigma_{y}}+\frac{\nu_{z}}{\sigma_{z}}\right)\right] \\
& p_{u u d}=\frac{1}{8}\left[1+\rho_{x y}-\rho_{y z}-\rho_{z x}+\Delta t\left(\frac{\nu_{x}}{\sigma_{x}}+\frac{\nu_{y}}{\sigma_{y}}-\frac{\nu_{z}}{\sigma_{z}}\right)\right] \\
& p_{u d u}=\frac{1}{8}\left[1-\rho_{x y}+\rho_{y z}-\rho_{z x}+\Delta t\left(\frac{\nu_{x}}{\sigma_{x}}-\frac{\nu_{y}}{\sigma_{y}}+\frac{\nu_{z}}{\sigma_{z}}\right)\right] \\
& p_{u d d}=\frac{1}{8}\left[1-\rho_{x y}-\rho_{y z}+\rho_{z x}+\Delta t\left(\frac{\nu_{x}}{\sigma_{x}}-\frac{\nu_{y}}{\sigma_{y}}-\frac{\nu_{z}}{\sigma_{z}}\right)\right] \\
& p_{d u u}=\frac{1}{8}\left[1-\rho_{x y}-\rho_{y z}+\rho_{z x}+\Delta t\left(-\frac{\nu_{x}}{\sigma_{x}}+\frac{\nu_{y}}{\sigma_{y}}+\frac{\nu_{z}}{\sigma_{z}}\right)\right] \\
& p_{d u d}=\frac{1}{8}\left[1-\rho_{x y}+\rho_{y z}-\rho_{z x}+\Delta t\left(-\frac{\nu_{x}}{\sigma_{x}}+\frac{\nu_{y}}{\sigma_{y}}-\frac{\nu_{z}}{\sigma_{z}}\right)\right] \\
& p_{d d u}=\frac{1}{8}\left[1+\rho_{x y}-\rho_{y z}-\rho_{z x}+\Delta t\left(-\frac{\nu_{x}}{\sigma_{x}}-\frac{\nu_{y}}{\sigma_{y}}+\frac{\nu_{z}}{\sigma_{z}}\right)\right] \\
& p_{d d d}=\frac{1}{8}\left[1+\rho_{x y}+\rho_{y z}+\rho_{z x}+\Delta t\left(-\frac{\nu_{x}}{\sigma_{x}}-\frac{\nu_{y}}{\sigma_{y}}-\frac{\nu_{z}}{\sigma_{z}}\right)\right]
\end{aligned}
$$

Since probability cannot be negative, Bayes' rule of probabilities that represent the joint process as the product of the marginal process for $x$ and the conditional process for $y$ as: $p(x, y)=p(y \mid x) p(x)$ is used. Similar approach is proposed by Hahn and Dyer (2008) and Abadie et al. (2013). Therefore, the conditional probabilities for $x$ are:

$$
\begin{aligned}
& p_{u}=\frac{1}{2}+\frac{1}{2} \sqrt{\Delta t} \frac{\nu_{x}}{\sigma_{x}} \\
& p_{d}=\frac{1}{2}-\frac{1}{2} \sqrt{\Delta t} \frac{\nu_{x}}{\sigma_{x}}
\end{aligned}
$$

It follows that $p_{u}+p_{d}=1$. Neither of $p_{u}$ or $p_{d}$ can be greater than one or less than zero. The probabilities are censored as follows:

$$
\begin{aligned}
& p_{u}^{*}=\max \left(0, \min \left(1, p_{u}\right)\right) \\
& p_{d}^{*}=1-p_{u}^{*}
\end{aligned}
$$

Now the conditional probabilities for $y$ can be derived as:

$$
\begin{aligned}
& p_{u / u}=\frac{p_{u u u}+p_{u u d}}{p_{u}} \\
& p_{d / u}=\frac{p_{u d u}+p_{u d d}}{p_{u}}
\end{aligned}
$$

These conditional probabilities exist only when $p_{u}^{*}>0$. If $p_{u}^{*}>0$, then $p_{u / u}+p_{d / u}=1$. Censoring 
these probabilities between 0 and 1 imply that

$$
\begin{aligned}
& p_{u / u}^{*}=\max \left(0, \min \left(1, p_{u / u}\right)\right) \\
& p_{d / u}^{*}=1-p_{u / u}^{*}
\end{aligned}
$$

If $p_{u / u}>0$, them the conditional probabilities for $z$ are:

$$
\begin{aligned}
& p_{u / u / u}=\frac{p_{\text {uuu }}}{p_{\text {uuu }}+p_{\text {uud }}} \\
& p_{d / u / u}=\frac{p_{\text {duu }}}{p_{\text {uuu }}+p_{\text {uud }}}
\end{aligned}
$$

Censoring these probabilities gives:

$$
\begin{aligned}
& p_{u / u / u}^{*}=\max \left(0, \min \left(1, p_{u / u / u}\right)\right) \\
& p_{d / u / u}^{*}=1-p_{u / u / u}^{*}
\end{aligned}
$$

Similarly,

$$
\begin{aligned}
& p_{u / d / u}^{*}=\max \left(0, \min \left(1, p_{u / d / u}\right)\right) \\
& p_{u / u / d}^{*}=\max \left(0, \min \left(1, p_{u / u / d}\right)\right) \\
& p_{u / d / d}^{*}=\max \left(0, \min \left(1, p_{u / d / d}\right)\right)
\end{aligned}
$$

In the end, the branch probabilities are:

$$
\begin{aligned}
& p_{u u u}^{*}=p_{u}^{*} \cdot p_{u / u}^{*} \cdot p_{u / u / u}^{*} \\
& p_{u u d}^{*}=p_{u}^{*} \cdot p_{u / u}^{*} \cdot p_{d / u / u}^{*} \\
& p_{u d u}^{*}=p_{u}^{*} \cdot p_{d / u}^{*} \cdot p_{u / d / u}^{*} \\
& p_{u d d}^{*}=p_{u}^{*} \cdot p_{d / u}^{*} \cdot p_{d / d / u}^{*} \\
& p_{d u u}^{*}=p_{d}^{*} \cdot p_{u / d}^{*} \cdot p_{u / u / d}^{*} \\
& p_{d u d}^{*}=p_{d}^{*} \cdot p_{u / d}^{*} \cdot p_{d / u / d}^{*} \\
& p_{d d u}^{*}=p_{d}^{*} \cdot p_{d / d}^{*} \cdot p_{u / d / d}^{*} \\
& p_{d d d}^{*}=p_{d}^{*} \cdot p_{d / d}^{*} \cdot p_{d / d / d}^{*}
\end{aligned}
$$




\section{Appendix D}

\section{Discretizing the Orenstein-Uhlenbeck}

\section{Process in an AR Process}

The mean-reverting Orenstein-Uhlenbeck process is equivalent to the discrete time autoregressive (AR) process The continuous time mean-reverting process is expressed as:

$$
d x=\kappa(\bar{x}-x) d t+\sigma d B
$$

After discretizing the above process:

$$
\begin{aligned}
\Delta x_{t} & =\kappa\left(\bar{x}-x_{t}\right)+\epsilon_{t}, \text { where } \epsilon_{t} \sim \sigma B_{t} \sim N\left(0, \sigma_{t}\right) \\
x_{t}-x_{t-1} & =\kappa\left(\bar{x}-x_{t}\right)+\epsilon_{t} \\
x_{t} & =\kappa \bar{x}-\kappa x_{t}+x_{t-1}+\epsilon_{t} \\
x_{t}+\kappa x_{t} & =\kappa \bar{x}+x_{t-1}+\epsilon_{t} \\
(1+\kappa) x_{t} & =\kappa \bar{x}+x_{t-1}+\epsilon_{t} \\
x_{t} & =(\kappa \bar{x})(1+\kappa)^{-1}+(1+\kappa)^{-1} X_{t-1}+\nu_{t} \\
x_{t} & =\alpha+\varrho x_{t-1}+\nu_{t}
\end{aligned}
$$

where $\alpha=(\kappa \bar{x})(1+\kappa)^{-1} ; \varrho=(1+\kappa)^{-1} ; \nu \sim N\left(0, \sigma_{t}(1+\kappa)^{-1}\right)$ (see Alexander $\left.(2008)\right)$. 


\section{Appendix E}

\section{Step size and Branch Probabilities by}

\section{Matching the Moments}

This Section shows an example of building a binomial lattice by matching the first and second moments of a geometric Brownian motion, $d x=\mu d t+\sigma d B$. Let us assume a lattice with an equal step size of up $(\Delta)$ and down $(-\Delta)$ movement.

Matching the first and second moments, respectively:

$$
\begin{array}{r}
p(\Delta)+(1-p)(-\Delta)=\mu \Delta t, \text { [Matching the first moment] } \\
p(\Delta)^{2}+(1-p)(-\Delta)^{2}-\mu^{2}(\Delta t)^{2}=\sigma^{2} \Delta t,[\text { Matching the second moment] }
\end{array}
$$

where, $p$ is the probability to move up and $(1-p)$ is the probability to move down.

From Equation (E.1), let us find $p$ :

$$
\begin{aligned}
p(\Delta)+(1-p)(-\Delta) & =\mu \Delta t \\
p \Delta-\Delta+p \Delta & =\mu \Delta t \\
2 p \Delta & =\Delta+\mu \Delta t \\
p & =\frac{\Delta+\mu \Delta t}{2 \Delta} \\
p & =\frac{1}{2}+\frac{\mu \Delta t}{2 \Delta}
\end{aligned}
$$


We replace this $p$ into Equation (E.2):

$$
\begin{aligned}
\frac{\Delta+\mu \Delta t}{2 \Delta} \times(\Delta)^{2}+\left[1-\frac{\Delta+\mu \Delta t}{2 \Delta}\right] \times(\Delta)^{2} & =\mu^{2}(\Delta t)^{2}+\sigma^{2} \Delta t \\
\frac{(\Delta+\mu \Delta t) \Delta}{2}+\frac{(2 \Delta-\Delta-\mu \Delta t) \Delta}{2} & =\mu^{2}(\Delta t)^{2}+\sigma^{2} \Delta t \\
\frac{(\Delta+\mu \Delta t) \Delta}{2}+\frac{2}{2} \Delta^{2}-\frac{(\Delta+\mu \Delta t) \Delta}{2} & =\mu^{2}(\Delta t)^{2}+\sigma^{2} \Delta t \\
\Delta^{2} & =\mu^{2}(\Delta t)^{2}+\sigma^{2} \Delta t \\
\Delta & =\sqrt{\mu^{2}(\Delta t)^{2}+\sigma^{2} \Delta t}
\end{aligned}
$$




\section{References}

Abadie, L.M. (2015). Operating flexibility at power plants: A market valuation. Electrical Power and Energy Systems, 64:41-49.

Abadie, L.M. and Chamorro, J.M. (2008a). Valuing flexibility: The case of an integrated gasification combined cycle power plant. Energy Economics, 30(4):1850-1881.

Abadie, L.M. and Chamorro, J.M. (2008b). European $\mathrm{CO}_{2}$ prices and carbon capture investments. Energy Economics, 30(6):2992-3015.

Abadie, L.M., Chamorro, J.M., and González-Egunio, M. (2013). Valuing uncertain cash flows from investments that enhance energy efficiency. Journal of Environmental Management, 116:113-124.

Ahrens, C.D. (2006). Meteorology Today: An Introduction to Weather, Climate, and Environment, Brooks/Cole, Belmont, CA, US.

Alaton, P., Djehiche, B., and Stillberger, D. (2002). On modeling and pricing weather derivatives. Applied Mathematical Finance, 9(1):1-20.

Alexander, C. (2008). Market Risk Analysis, Vol. I $\&$ II, Wiley, Chichester, UK.

Amram, M. and Kulatilaka, N. (1996) Real Options: Managing Strategic Investment in an Uncertain World, Harvard Bussiness School Press, Boston, MA, US.

Andrew, A. and Bekaert, G. (2002). Regime switches in interest rates. Journal of Business and Economic Statistics, 20(2):163-182.

Benth, F.E. and Šaltytė-Benth, J. (2005). Stochastic modelling of temperature variations with a view towards weather derivatives. Applied Mathematical Finance, 12(1):53-85.

Benth, F.E. and Šaltytė-Benth, J., 2007. The volatility of temperature and pricing of weather derivatives. Quantitative Finance, 7(5):553-561.

Benth, F.E., Šaltytė-Benth, J. and Koekebakker, S. (2007). Putting a price on temperature. Scandinavian Journal of Statistics, 34(4):746-767. 
Benz, E. and Trück, S. (2009). Modeling the price dynamics of $\mathrm{CO}_{2}$ emission allowances. Energy Economics, 31(1):4-15.

Bierbrauer, M., Trück S., and Weron, R. (2004). Modelling electricity prices with regime-switching models. Lecture Notes in Computer Science, 3039:859-867.

Bollen, N.P.B. (1998). Valuing options in regime-switching models. The Journal of Derivatives, 6(1):38-49.

Bollen, N.P.B., Gray, S.F., and Whaley R.E. (2000). Regime-switching in foreign exchange rates: Evidence from currency option prices. Journal of Econometrics, 94:239-276.

Boyce, M.P. (2006). Gas Turbine Engineering Handbook, Elsevier. Retrieved on March 23, 2010, http://www.knovel.com.

Boyle, P.P. (1988). A lattice framework for option pricing with two state variables. Journal of Financial and Quantitative Analysis, 23(1):1-12.

Boyle, P.P., Evnine, J., and Gibbs, S. (1989). Numerical evaluation of multivariate contingent claims. The Review of Financial Studies, 2(2):241-250.

Brody, D.C., Syroka, J., and Zervos, M., 2002. Dynamical pricing of weather derivatives. Quantitative Finance, 2(3):189-198.

Brooks, C. (2008). Introductory Econometrics for Finance. Cambridge University Press, Cambridge, UK.

Brooks, C. (2009). RATS Handbook to Accompany Introductory Econometrics for Finance. Cambridge University Press, Cambridge, UK.

Campbell, S.D. and Diebold, F.X. (2005). Weather forecasting for weather derivatives. Journal of the American Statistical Association, 100:6-16.

Cao, M. and Wei, J. (2004). Weather derivatives valuation and market price of weather risk. The Journal of Futures Markets, 24(11):1065-1089.

Carmona, R. and Hinz, J. (2011). Risk-neutral models for emission allowance prices and option valuation. Management Science, 57(8):1453-1468.

Cassano, M. and Sick, G. (2013). Valuation of a spark spread: An LM6000 power plant. The European Jouranl of Finance, 19(7-8):689-714. 
Chen, F.Y. and Yano, C.A. (2010). Improving supply chain performance and managing risk under weather-related demand uncertainty. Management Science, 56(8):1380-1397.

Chesney, M. and Taschini, L. (2012). The endogenous price dynamics of emission allowances and an application $\mathrm{CO}_{2}$ option pricing. Applied Mathematical Finance, 19(5):447-475.

Clewlow, L. and Strickland, C. (1998). Implementing Deivatives Models. Wiley, Chichester, UK.

Considine, G. (1999). Introduction to weather derivatives. Weather Derivatives Group, Aquila Energy. Available at www.cmegroup.com/trading/weather/files/WEA_intro_to_weather_der. pdf (accessed on September 10, 2009).

Cox, J.C., Ross, S.A., and Rubinstein, M. (1979). Option pricing: A simplified approach. Journal of Financial Economics, 7:229-263.

Coulon, M. (2013). Additional Notes: Introduction to Commodities and Reduced-Form Price Models. Avalaible at https://orfe.princeton.edu/rtg/fmsummer/sites/orfe.princeton.edu. rtg.fmsummer/files/Coulon_summerschoolnotes.pdf (accessed on May 5, 2015).

Davis, M. (2001). Pricing weather derivatives by marginal value. Quantitative Finance, 1:1-4.

Dixit, A.K. and Pindyck, R.S. (1994). Investement Under Uncertainty, Princeton University Press, Princeton, NJ, US.

Deng, S., Johnson, B., and Sogomonian, A. (2001). Exotic electricity options and the valuation of electricity generation and transmission assets. Decision Support Systems, 30:383-392.

Deng, S. and Oren, S. (2006). Electricity derivatives and risk management. Energy, 31:940-953.

Dornier, F. and Queruel, M. (2000). Caution to the wind. Energy \& Power Risk Management, $13(8): 30-32$.

Dorfleitner, G. and Wimmer, M. (2010). The pricing of temperature futures at the Chicago Mercantile Exchange. Journal of Banking \& Finance, 34:1360-1370.

EIA (2010). International energy outlook. Energy Information Admistration Report. http: //large.stanford.edu/courses/2010/ph240/riley2/docs/EIA-0484-2010.pdf (accessed on May 27, 2015).

EIA (2013). International energy outlook. Energy Information Admistration Report. http://www . eia.gov/forecasts/ieo/electricity.cfm (accessed on August 27, 2014). 
EIA (2015). U.S. Energy Information Admistration Report. http://http://www. eia.gov/tools/ faqs $/$ faq. $c f m ? i d=65 \backslash \& t=2$ (accessed on February 2, 2015).

Elias, R.S., Wahab, M.I.M., and Fang, L. (2014). A comparison of regime-switching temperature modeling appraoches for applications in weather derivatives. European Journal of Operational Research, 232(3):549-560.

Enders, W. (2003). RATS Programming Manual. http://www.estima.com/enders.

Environment Canada, 2009. http://www.climate.weatheroffice.gc.ca/climateData/canada_ e.html (accessed on September 10, 2009).

Eydeland, A. and Wolyniec, K. (2003). Energy and Power Risk Management: New Developments in Modeling, Pricing and Hedging, Wiley, Hoboken, NJ.

Fiorenzani, S. (2006). Quantitative Methods for Electricity Trading and Risk Management: Advanced Mathematical and Statistical Methods for Energy Finance, Palgrave Macmillan, NY, US.

Fleten, S. and Näsäkkälä, E. (2009). Gas-fired power plants: Investment timing, operating flexibility and $\mathrm{CO}_{2}$ capture. Energy Economics, 32(4):805-816.

Francis, D. and Hengeveld, H. (1998). Extreme weather and climate change, Atmospheric Environment Service, Environment Canada, 1998. http://www.geology.iastate.edu/gccourse/ history/trends/ExtremeWxClim.pdf (accessed on April 20, 2011).

Gardner, D. and Zhuang, Y. (2000). Valuation of power generation assets: A real options approach. Algorithmic Research Quarterly, 3(3):9-20.

Geman, H. (2000). The Bermuda triangle: Weather, electricity, and insurance derivatives. The Journal of Alternative Investments, 3(1):61-69.

Green, R. (2008). Carbon tax or carbon permits: The impact on generator's risk. The Energy Journal, 29(3):67-89.

Hahn, W.J. and Dyer. J.S. (2008). Discrete time modeling of mean-reverting stochastic processes for real option valutaion. European Journal of Operational Research, 184(2):534-548.

Hamilton, J.D. (2008). Regime-switching models. In New Palgrave Dictionary of Economics, 2nd edtition, S. Durlauf and L. Blume (Eds.), Palgrave McMillan, NY, US.

Heydari, S., and Siddiqui, A. (2010). Valuing a gas-fired plant: A comparison of ordinary linear models, regime-switching appraoches, and models with stochastic volatility. Energy Economics, 32:709-725. 
Higgs, H. and Worthington, A. (2008). Stochastic price modeling of high volatility, mean-reverting, spike-prone commodities: The Australian wholesale spot electricity market. Energy Economics, 30:3172-3185.

Hsu, M. (1998). Spark spread options are hot, The Electricity Journal, 11(2):28-39.

Huang, Y. (2010). The price dynamics in the emission market and the valuation of allowance derivatives, Working paper, www.ssrn.com/abstract=1575617 (accessed on June 12, 2014).

Huisman, R. (2008). The influence of temperature on spike probability in day-ahead power prices. Energy Economics, 30(5):2697-2704.

Huisman, R. and Mahieu, R. (2003). Regime jumps in electricity prices. Energy Economics, 25:425434.

Hull, J.C. (2002). Options, Futures and Other Derivatives, 5th edition, Prentice Hall, Upper Saddle River, NJ, US.

Jewson, S. and Brix, A. (2005). Weather Derivative Valuation: The Meteorological, Statistical, Financial and Mathematical Foundations. Cambridge University Press, Cambridge, UK.

Johnson, B. and Miranda, M.I.D. (2003). Modelling generation assets. In Real Options and Energy Management: Using Options Methodology to Enhance Capital Budgeting Decisions, Ronn, E.I. (ed), Risk Books, London, UK, pp. 393-427.

Ku, A. (2001). Betting on weather. Global Energy Business. July/August issue, p 28.

Li, L. and Kleindorfer, P.R. (2009). On hedging spark spread options in electricity markets. Risk and Decision Analysis, 1:211-220.

Linn, J., Muehlenbachs, L. and Wang, Y. (2014). How do natural gas prices affect electricity consumers and the environment? Resources for the Future Discussion Paper No. 14-19. Available at SSRN: http://ssrn. com/abstract=2537833 (accessed on August 28, 2015).

Liu, W.-H. and Chyi, Y.-L. (2006). A Markov regime-switching model for the semiconductor industry cycles. Economic Modelling, 23(4):569-578.

London, J., 2007. Modeling Derivatives Applications in Matlab, $C++$, and Excel. FT Press, Upper Saddle River, NJ, US.

Margrabe, W. (1978). The value of an option to exchange one asset for another. Journal of Finance, $33(1): 177-186$. 
McKinnon, R.I. (1967). Futures markets, buffer stocks, and income stability for primary producers. Journal of Political Economy, 75(6):844-861.

Mnif, W. and Davison, M. (2011). Carbon emission markets. In Quantitative Financial Risk Management, Wu, D.D. (ed.), Springer-Verlag, Heidelberg, Germany.

Mohsin, T. and Gough, W.A. (2010). Trend analysis of long-term temperature time series in the Greater Toronto Area (GTA). Theoretical and Applied Climatology, 101(3-4):311-327.

Näsäkkälä, E. and Fleten, S. (2005). Flexibility and technology choice in gas fired power plant investments. Review of Financial Economics, 14:371-393.

Nelson, D.B. and Ramaswamy, K. (1990). Simple binomial processes as diffusion approximations in financial models. The Review of Financial Studies, 3(3):393-430.

Øksendal, B. (2000). Stochastic Differential Equations: An Introduction with Applications, 5th ed. Springer-Verlag, NY, US.

Pilipovic, D. (2007). Energy Risk: Valuing and Managing Energy Derivatives, McGraw-Hill, NY, US.

Rogers, J. (2002). Strategy, Value and Risk: The Real Options Approach, Palgrave, Basingstoke, Hampshire, UK.

Sa, A.D., and Zubaidy, S. (2011). Gas turbine performance at varying ambient temperature. Applied Thermal Engineering, Vol. 31(14-15):2735-2739.

Schwartz, E. (1997). The stochastic behavior of commodity prices: Implications for valuation and hedging. The Journal of Finance, 52(3):923-973.

Schwartz, E. and Smith, J.E. (2000). Short-term variations and long-term dynamics in commodity prices. Management Science, 46(7):893-911.

Seifert, J., Uhrig-Homburg, M., and Wagner, M.W. (2008). Dynamic behavior of $\mathrm{CO}_{2}$ spot prices. Journal of Environmental Economics and Management, 56(2):180-194.

Shimko, D. (1994). Options on futures spreads: hedging, speculation, and valuation. The Jourmnal of Futures Market, 14(2):183-213.

Teetor, P. (2011). R Cookbook, O‘Reilly, Sebastopol, CA, US. 
Thornton, P. (2011). Bad weather around the world hits UK companies, Financial Director, Incisive Financial Publishing Limited, Haymarket House, London, UK, 18 February, 2011. http://www.financialdirector.co.uk/financial-director/analysis/2027283/ bad-weather-world-hits-uk-companies (accessed on March 10, 2011).

Trigeorgis, L. (1996). Real Options: Managerial Flexibility and Strategy in Resource Allocation. The MIT Press, Cambridge, MA, US.

Tseng, C. and Barz, G. (2002). Short-term genartion asset valuation: A real option approach. Operations Research, 50(2):297-310.

Tseng, C. and Lin, G. (2007). A framework using two-factor price lattices for generation asset valuation. Operations Research, 55(2):234-251.

Tseng, C., Zhu, W., and Dmitriev, A. (2009). Variable capacity utilization, ambient temperature shocks and generation asset valuation. Energy Economics, 31(6):888-896.

Wahab, M.I.M. and Lee, C.-G. (2009). A lattice approach to pricing of multivariate contingent claims with regime switching. The Journal of Derivatives, 17(1):49-61.

Wahab, M.I.M., Yin, Z., and Edirisinghe, N. (2010). Pricing swing options in the electricity markets under regime-switching uncertainty. Quantitative Finance, 10(9):975-994.

Wang, C-H., and Min, K.J. (2013). Electric power plant valuation based on day-ahead spark spreads. The Engineering Economist, 58(3):157-178.

Weron, R. (2007). Modeling and Forecasting Electricity Loads and Prices: A Statistical Approach. Wiley, Chichester, UK.

Wood, A.J. and Wollenberg, B.F. (1996). Power Generation, Operation and Control, Wiley, NY, US.

WRMA (Weather Risk Management Association) (2003). Weather Risk Management Survey: Contracts Beginning in the 12 Months after March 2002. Available at http://www . wrma.org/wrma/ library/2003SurveyResults.pdf (accessed on March 9, 2009). 